\title{
Article
}

\section{Impact of liquid lipid on development and stability of trimyristin nanostructured lipid carriers for oral delivery of resveratrol}

Houacine, Chahinez, Adams, David R and Singh, Kamalinder

Available at http://clok.uclan.ac.uk/34006/

Houacine, Chahinez, Adams, David $R$ and Singh, Kamalinder ORCID: 00000001-7325-0711 (2020) Impact of liquid lipid on development and stability of trimyristin nanostructured lipid carriers for oral delivery of resveratrol. Journal of Molecular Liquids, 316 . ISSN 0167-7322

It is advisable to refer to the publisher's version if you intend to cite from the work. http://dx.doi.org/10.1016/j.molliq.2020.113734

For more information about UCLan's research in this area go to http://www.uclan.ac.uk/researchgroups/ and search for <name of research Group>.

For information about Research generally at UCLan please go to http://www.uclan.ac.uk/research/

All outputs in CLoK are protected by Intellectual Property Rights law, including Copyright law. Copyright, IPR and Moral Rights for the works on this site are retained by the individual authors and/or other copyright owners. Terms and conditions for use of this material are defined in the policies page. 


\section{Impact of liquid lipid on development and stability of trimyristin nanostructured lipid}

carriers for oral delivery of resveratrol

Chahinez Houacine $^{1}$, David Adams ${ }^{1}$ and Kamalinder. K Singh ${ }^{1,2,3 *}$

${ }^{1}$ School of Pharmacy and Biomedical Sciences, Faculty of Clinical and Biomedical Sciences, University of Central Lancashire, Preston, PR1 2HE, United Kingdom

${ }^{2}$ UCLan Research Centre of Smart Materials, University of Central Lancashire, Preston, PRI 2HE, United Kingdom

${ }^{3}$ UCLan Research Centre of Drug Design and Development, University of Central Lancashire, Preston, PR1 2HE, United Kingdom

*To whom correspondence should be addressed:

Kamalinder K. Singh

School of Pharmacy and Biomedical Sciences

Faculty of Clinical and Biomedical Science

University of Central Lancashire

Preston, PR1 2HE, United Kingdom

Email: ksingh1@uclan.ac.uk; kksingh35@hotmail.com 


\section{Abstract}

Nanostructured lipid carriers (NLCs) have emerged as versatile carriers to improve oral bioavailability of poorly water-soluble drugs as well as to protect labile drugs from degradation and metabolism. Prepared by blending solid and liquid lipids, the choice of liquid lipid can have a great influence on their physicochemical characteristics and stability. The present work investigated the impact of six different liquid lipids with diverse chemical structures and hydrophilic and lipophilic balance (HLBs) on the critical quality attributes (CQAs) and storage stability of NLCs with trimyristin as solid lipid. Resveratrol (RES) was used as model drug as its low water solubility, poor bioavailability, rapid metabolism and clearance from systemic circulation restricts its clinical use despite its wide spectrum of biological activities. Liquid lipids investigated included, two triglycerides, one medium chain (C8) glycerol tricaprylate (GTC) and second, long chain (C18) glyceryl trioleate (GTO); two propylene glycol fatty acid esters, propylene glycol monocaprylate (PGMC) and propylene glycol monolaurate (PGML); fatty acid ester decyl oleate (DO) and a PEGylated lipid polyethylene glycol-8 caprylic/capric glycerides (PCG). Box-Behnken experimental design was employed to ascertain the effect of four independent factors viz. type of liquid lipid, amount of liquid lipid, amount of drug and surfactant concentration and interactions between these factors on the CQAs of NLCs as response variables viz. particle size (PS), polydispersity index, (PDI) zeta potential (ZP), drug encapsulation efficiency (EE), and drug loading (DL). The relationship between various factors and responses was established by response surface methodology (RSM). The oils with higher lipophilicity C18 triglycerides (GTO) and C18 fatty acid ester DO yielded NLCs with lower PS as compared to the oils with lower lipophilicity (PGC, PGMC and PGML). Although increasing the concentration of liquid lipids had an upward trend on the PS of NLCs, its PDI was more predominantly influenced by the nature of liquid lipid. The characteristic of the liquid lipid influenced the DL remarkably which varied from 2.94 to $7.56 \%$. The ZP of nanoparticles varied from -21.3 to $-39.9 \mathrm{mV}$ with liquid lipids with free hydroxyl groups and higher HLB playing a more prominent role contributing to the increase in the negative surface charge. The characteristics of liquid lipid influenced the depression of melting point of RES with maximum distortion of the crystal lattice was caused by PGMC and least by GTO. The two, long chain oleates, DO and GTO exhibited a shift of lipid peak in NLCs to higher melting points (116 and $\left.111^{\circ} \mathrm{C}\right)$ than the less lipophilic liquid lipids $\left(103-104^{\circ} \mathrm{C}\right)$. The attributes of liquid lipid also discriminate whether the particle growth during storage followed Oswald's ripening or coalescence. NLC containing GTO exhibited the highest stability in terms of maintenance of the PS and particle size distribution at $20^{\circ} \mathrm{C}$. This study provides vital insight on impact of 
liquid lipids and future strategy for rational design of stable NLC systems for delivery of various bio-actives for drug delivery applications.

\section{Key words}

Nanostructured lipid carriers, Trimyristin, Liquid lipid, Resveratrol, Box-Behnken design, Stability of Nanostructured lipid carriers. 


\section{Introduction}

There has been tremendous increase in studies involving nanostructured lipid carriers (NLCs) in the last decade. NLCs have emerged as effective and prominent alternative nanocarrier to emulsions, liposomes, solid lipid nanoparticles (SLNs) and their polymeric counterparts due to their numerous distinct advantages [1-3]. NLCs are promising delivery systems for carrying drugs to their site of action while protecting them from the degradation in the external environment $[4,5]$. Their amicable physicochemical and biopharmaceutical properties have been widely studied for oral delivery of small and large molecules alike [6].

NLCs were typically developed in order to overcome the potential problems associated with solid lipid nanoparticles (SLN); which are limited in their drug loading capacity and potential drug expulsion during storage. Prepared from blend of solid and liquid lipids, NLCs have imperfect crystal structure owing to the presence of liquid lipid which allow more drug incorporation in their lipid matrix [7]. The diversity in chemistry and structural richness of lipids offer a wide spectrum to fabricate the NLCs with different characteristics and containing diverse active molecules. However, stability of NLCs is strongly affected by the type and the amount of liquid oil in the lipid matrix. Dispersion stability is enhanced when the oil concentration is increased, which leads to the decrease in the crystallization and melting point of the lipid matrix, polymorphic transformation rate is also increased that ends up with particles having more spherical shape, thus, particles retain their sphericity over longer period of time, accordingly, enhancing their suspension stability [8]. Thus, selection of liquid lipid is critical to the performance and stability of NLCs. Understanding the chemistry and physicochemical properties of the lipids is a prerequisite for successful formulation of stable NLCs with predictable biopharmaceutical properties. Though, researchers have employed myriad of liquid lipids [9] to prepare NLCs but there are a few reports of a direct comparison of the effect of liquid lipids on the quality parameters and stability of NLCs. Also little is known on the effect of type of liquid oil on the polymorphic transformations and lipid modification or interactions within NLCs [10].

Resveratrol (RES) (3,4',5-trans-trihydroxystilbene) is a naturally occurring phytoalexin which shows a wide spectrum of biological activity, including anti-tumour, anti-inflammatory, cardio and neuroprotective with anti-oxidative effects. RES is found to occur in red grapes, peanut (Arachis hypogaea) sprouts, polygonum and many other species and has also been also been 
recommended as a nutraceutical for its various beneficial actions [11]. Although range of promising biological effects of RES have been demonstrated, its clinical application is limited because of its low systemic availability and rapid clearance from the circulation [12]. Moreover, its low water solubility reduces the dissolution-rate limited cell absorption which further coupled with rapid degradation in gastro-intestinal tract leads to reduced oral bioavailability [13]. The therapeutic potential of RES can be realized in vivo for any clinical effect, only if the limitations bound to its bioavailability are overcome. NLCs present as promising drug delivery system, which could overcome bioavailability problems of RES with improved absorption and protection against rapid metabolism.

The aim of the present study is to investigate the effect of six different liquid lipids with varying chemical compositions, molecular structures and hydrophilic lipophilic balance (HLB) on the critical quality attributes (CQAs) and stability of NLCs with RES as a model drug (Table1). Two triglycerides, one medium chain, glycerol tricaprylate (GTC) and second long chain, glyceryl trioleate (GTO), two propylene glycol fatty acid esters, propylene glycol monocaprylate (PGMC) and propylene glycol monolaurate (PGML), one fatty acid ester, decyl oleate (DO) and one PEGylated lipid polyethylene glycol-8 caprylic/capric glycerides (PCG) were employed along with trimyristin as solid lipid for preparation of NLCs using hot melt high pressure homogenization technique. Box-Behnken experimental design was employed to examine the effect of four independent factors viz. type of liquid lipid, amount of liquid lipid, amount of drug and surfactant concentration and interactions between these factors on the CQAs of NLCs as response variables viz. particle size (PS), polydispersity index (PDI), zeta potential (ZP), drug encapsulation efficiency (EE), and drug loading (DL). The relationship between various factors and responses was established by response surface methodology (RSM) [14, 15]. Detailed thermal analysis was carried out using differential scanning calorimetry (DSC) to determine the effect of liquid lipids on the solid lipid as physical mixture and within the NLC formulation as well as on drug solubilisation. The effect of the liquid lipids was also evaluated on the storage stability of the NLCs over the period of six months by observing the changes in the PS, PDI, ZP, EE and DL and its ramification on mechanism of particle growth was also ascertained. 


\section{Materials and methods}

\subsection{Materials}

The following materials were used from the determined sources without further purification. Trimyristin (Dynasan 114) was kindly donated as a gift sample from Cremer oleo division, PEG-8 caprylic/capric Glyceride (Labrasol), propylene glycol monolaurate (Lauroglycol 90), propylene glycol monocaprylate (type II) NF (Capryol 90) were kindly supplied by Gattefosse. Glyceryl trioleate was purchased from Sigma-Aldrich, glycerol tricaprylate (Miglyol 808) was was generously supplied by Cremer and decyl oleate were kindly gifted by BSAF. Sodium cholate, Tween 80 and Span 80 were purchased from Sigma-Aldrich, UK. Lipoid S75, E80 and Phospholipon $90 \mathrm{H}$ was kind gift from Lipoid oleo division, Germany. Amicon $0.5 \mathrm{~mL}$ centrifugal tubes (3K Da molecular weight cut-off) was purchased from Spectrum Labs, USA. Resveratrol was purchased from Manchester Organics, UK. High performance liquid chromatography (HPLC) solvents and water were purchased from Fisher Scientific, UK. All other chemicals were of analytical grade. Purified water was used throughout the study.

\subsection{Measurement solubility of resveratrol in liquid lipids}

RES solubility in each of the six liquid lipids was determined by adding an excess amount of drug (approximately $200 \mathrm{mg}$ ) to each of the individual liquid lipid $(5 \mathrm{~mL})$ in screw-capped bottle [16]. After $24 \mathrm{~h}$ of stirring, the sample was centrifuged at 13,000 rpm for 5 minutes and the clear supernatant layer was diluted with the mobile phase (acetonitrile: water) and analysed by a validated reverse phase HPLC method using C18 Luna column and detected at $306 \mathrm{~nm}$ [17].

\subsection{Preparation of resveratrol loaded NLCs (RES-NLCs)}

NLCs were prepared by hot melt homogenisation process. Briefly, a hot aqueous phase containing Tween 80 and sodium cholate $(0.25 \%)$ was preheated to $70^{\circ} \mathrm{C}$. Trimyristin was melted and either of the six liquid lipids PCG, PGMC, PGML, GTO, DO or GTC were subsequently added to it. RES was solubilized in mixture of soy phospholipid (S75), egg phospholipid (E80) both at a concentration of $0.1 \% \mathrm{w} / \mathrm{w}$ and Phospholipon $90 \mathrm{H}(0.3 \% \mathrm{w} / \mathrm{w})$. This drug phospholipid mixture was added to the molten lipid phase. The resultant mix was left for continued mixing to get a uniform dispersion of lipid phase. The lipid phase was added to the preheated aqueous phase in a drop wise manner and homogenized for 10 minutes on a T25 basic Ultra-Turrax (IKA England Ltd). The resultant hot o/w nanoemulsion was 
homogenized using high pressure homogenizer Nano DEE BEE (BEE International. USA) at 10,000, 20,000 and 30,000 bar for 3, 3 and 5 cycles, respectively. The nanodispersion was left to cool at room temperature $\left(20^{\circ} \mathrm{C}\right)$ for the re-crystallization of the lipid to yield six types of NLCs viz. RES-NLC-GTO, RES-NLC-GTC, RES-NLC-PCG, RES-NLC-PGMC, RES-NLCPGML and RES-NLC-DO. Blank NLCs (B-NLCs) without the drug were prepared using the exact procedure mentioned above but without incorporation of RES for each of the liquid lipids.

\subsection{Optimization by Box and Behnken design}

To investigate the effect of six different liquid lipids on the formulation of NLCs, 4-factor, 3level Box-Behnken design was employed to evaluate the main, interaction and quadratic effects of four independent variables at three levels on the five identified CQAs as dependent variables. Four critical independent variables were investigated namely; type of liquid lipid (D), concentration of liquid lipid $\left(\mathrm{X}_{1}\right)$, Tween 80 concentration $\left(\mathrm{X}_{2}\right)$, and amount of $\operatorname{drug}\left(\mathrm{X}_{3}\right)$. CQA is a physical, chemical, biological, or microbiological property or characteristic that should be within an appropriate limit, range, or distribution to ensure the desired product quality [18]. PS, PDI, ZP, EE and DL being the most influential parameters, which characterize the nanostructured drug delivery system were determined as CQAs and taken as response variables [19]. Table 2 shows the experimental design points with variables coded values (low, medium, and high) used in matrix of experiments with thirteen runs executed for each of the six liquid lipids totalling to 78 runs. Two-dimensional contour plots composed of the relation between two factors while the third factor being kept constant were generated by the Design Expert ${ }^{\circledR}$ software (Stat-Ease Design-Expert trial Version 9.0.4.1). The linear correlation plots of the actual experimental values were compared to the corresponding predicted values for each response for optimum model validation. The regression equation describing the effects of the variables on the responses in terms of linear, interactive and quadratic models was generated as given below:

$\mathbf{Y}=\mathbf{b}_{0}+\mathbf{b}_{1} \mathbf{X}_{1}+\mathbf{b}_{2} \mathbf{X}_{2}+\mathbf{b}_{3} \mathbf{X}_{3}+\mathbf{b}_{12} \mathbf{X}_{1} \mathbf{X}_{2}+\mathbf{b}_{13} \mathbf{X}_{1} \mathbf{X}_{3}+\mathbf{b}_{23} \mathbf{X}_{2} \mathbf{X}_{3}+\mathbf{b}_{11} \mathbf{X}_{1}{ }^{2}+\mathbf{b}_{22} \mathbf{X}_{2}{ }^{2}+\mathbf{b}_{33} \mathbf{X}_{3}{ }^{2}$

\section{Equation 1}

Where $\mathrm{Y}$ is the measured response associated with each factor level combination; $\mathrm{b}_{0}$ is an intercept; $b_{11}-b_{33}$ are regression coefficients computed from the observed experimental $Y$ values; and $\mathrm{X}_{1}, \mathrm{X}_{2}$, and $\mathrm{X}_{3}$ are the coded levels of independent variables. The terms $\mathrm{X}_{1} \mathrm{X}_{2}$ and $\mathrm{X}_{\mathrm{i}}^{2}(\mathrm{i}=1,2$, or 3$)$ represent the interaction and quadratic terms, respectively [20]. The six optimized formulations with six different liquid lipid compositions were selected on the bases 
of desirability functions and subjected to detailed physicochemical characterization and stability studies.

\subsection{Physicochemical characterization of RES-NLCs}

\subsubsection{Particle size and polydispersity index}

The PS and PDI were measured using dynamic light scattering technique on a Malvern Zetasizer (Nano ZS, Malvern Ltd., Worcestershire, UK). Disposable polystyrene cells having $10 \mathrm{~mm}$ diameter were employed to measure hydrodynamic diameter (z-average) and PDI. All measurements were repeated three times for all the values at an angle of $173^{\circ}$ at $25^{\circ} \mathrm{C}$.

\subsubsection{Zeta potential measurements}

$\mathrm{ZP}$ reflects the electric charges on the particle surface. It is considered as a valuable parameter to predict the physical stability of colloidal system. ZP was determined by the measurement of the electrophoretic mobility using Malvern Zetasizer Nano ZS (Malvern Instruments, UK).

\subsection{Entrapment efficiency and drug loading}

The amount of free drug was determined by ultrafiltration method with $3 \mathrm{KDa}$ molecular weight cut-off. Briefly, RES-NLC dispersion was placed into centrifugal filter tube and centrifuged at $13000 \mathrm{rpm}$ for $60 \mathrm{~min}$. After centrifugation, the amount of soluble free drug in the aqueous phase was detected by HPLC method at $306 \mathrm{~nm}$. The total drug in NLCs was determined by adding $4 \mathrm{~mL}$ of tetrahydrofuran to $1 \mathrm{~mL}$ of nanoparticle dispersion to ensure that the lipid and the drug were completely dissolved. The solution was filtered through $0.22 \mu \mathrm{m}$ filter diluted with the mobile phase and analyzed by HPLC. The amount of drug encapsulated in NLCs was determined by the indirect method as the difference between total drug in the NLCs and free drug. This method is simple and widely used for determining the EE [14]. EE (\%) and DL (\%) were calculated as follow:

\section{EE $(\%)=\underline{W}_{\text {total }}-W_{\text {free }} \times \mathbf{1 0 0} \% \quad$ Equation 2 \\ DL $(\%)=\underline{W}_{\text {total }}-W_{\text {free }} \times \mathbf{1 0 0} \% \quad$ Equation 3 \\ $\mathbf{W}_{\text {Lipids }}$}

where $\mathrm{W}_{\text {free }}$ is the amount of free drug in the supernatant; $\mathrm{W}_{\text {total }}$ amount of RES added; $\mathrm{W}_{\text {lipids }}$ is the total amount of lipids in the formulation. 


\subsection{Differential scanning calorimetry (DSC)}

To discern the interaction between different liquid lipids and solid lipid; and liquid lipids and RES, DSC was performed on differential scanning calorimeter (MetllerToledo $823^{\mathrm{e}}$ instruments, Switzerland) measuring the melting enthalpy of bulk RES, trimyristin, physical mixture of trimyristin with different liquid lipids at three concentrations, physical mixture of RES with different liquid lipids also at the three concentrations. Samples were placed in a flat bottom $40 \mu \mathrm{L}$ aluminum pan and covered and sealed with pin-holed aluminum lid (Mettler Toledo, UK). An empty aluminum pan was employed as the reference and each thermograph was baseline corrected. In order to study the effect of liquid lipid addition on the thermal behavior of the solid lipid, the heating runs were performed on physical mixtures from $25^{\circ}$ to $70^{\circ} \mathrm{C}$, the heating rate was $10^{\circ} \mathrm{C} / \mathrm{min}$, with a nitrogen purge of $0.2 \mathrm{~mL} / \mathrm{min}$., then kept isothermal at $70^{\circ} \mathrm{C}$ for $60 \mathrm{~min}$ followed by cooling down to $0^{\circ} \mathrm{C}$ at a rate of $10^{\circ} \mathrm{C} / \mathrm{min}$, kept isothermal at $0^{\circ} \mathrm{C}$ for $2 \mathrm{~min}$ then heated again up to $300^{\circ} \mathrm{C}\left(10^{\circ} \mathrm{C} / \mathrm{min}\right)$.

For DSC studies on NLC formulations, the sample was accurately weighed and the heating runs were started from $25^{\circ}$ to $300^{\circ} \mathrm{C}$ at scan rate of $10^{\circ} \mathrm{C} / \mathrm{min}$ with a nitrogen purge of $0.2 \mathrm{~mL} / \mathrm{min}$, then cooled down to $0^{\circ} \mathrm{C}$, kept isothermal at $0^{\circ} \mathrm{C}$ for $2 \mathrm{~min}$ then heated to $300^{\circ} \mathrm{C}$ with heating rate of $10^{\circ} \mathrm{C} / \mathrm{min}$. The resulting thermograms were analyzed using Mettler StarE DB V9.10 software and comparison of melting enthalpy/g of the individual materials with the melting enthalpy/g of the NLC dispersion was made to estimate the rate of crystallinity of the sample.

\subsection{Storage stability}

Stability of the six optimized NLC formulations was evaluated for a period of six months. Briefly, RES-NLC-GTO, RES-NLC-GTC, RES-NLC-PCG, RES-NLC-PGMC, RES-NLCPGML and RES-NLC-DO were prepared and stored in sealed amber colour glass bottles at both $4^{\circ} \mathrm{C}$ and $20^{\circ} \mathrm{C}$. Samples were withdrawn at one, three and six month intervals and characterized for PS, PDI , ZP \%EE and \%DL [21].

\subsection{Statistical analysis}

For the Box and Benkhen design, Analysis of Variance (ANOVA) provision available in the software (Stat-Ease Design-Expert trial Version 9.0.4.1) was used to establish the statistical validation of the polynomial equations generated by Design expert ${ }^{\circledR}$ between the dependent and the independent variables at $95 \%(p$-value $=0.05)$ significance level. All stability study 
results were expressed as a mean \pm SD. Two-way ANOVA followed by Bonferroni post-test was carried out to compare replicates $(n=3)$ using the GraphPad Prism 5 software. Difference at $\mathrm{p}<0.05$ was considered statistically significant.

\section{Results and discussion}

\subsection{Solubility of resveratrol in different liquid oils}

The drug carrying capacity of NLCs is directly dependent on the solubility of the respective drug in the lipid matrix and is one the of important determinant for the development of an NLC formulation. The drug should have high solubility in the lipids in order to achieve maximum entrapment in the NLC matrix and also prevent any crystallisation of drug during storage [22]. RES exhibited highest solubility $(112.28 \pm 6.81 \mathrm{mg} / \mathrm{mL})$ in PCG (Labrasol) (Figure 1) which consists of 30\% mono-,di- and triglycerides of C 8 and $\mathrm{C} 10$ fatty acids, $50 \%$ of mono- and diesters of polyethylene glycol, 20\% of free PEG 400 with high HLB of 14 [23]. Due to the ability of the long PEG chains to uphold more drug and high HLB value of Labrasol lead to its higher solubilizing capacity for RES. Labrasol as an excipient is known to increase the solubility and bioavailability of hydrophilic and hydrophobic drugs [24]. Hydrophobic liquid oils, GTO and DO, both esters of long chain fatty acid oleic acid with low HLB values showed least solubility for RES. The solubility in different carriers followed the pattern from order of high to low solubility of RES in various liquid lipid $\mathrm{PGC}>\mathrm{PGML}>\mathrm{PGMC}>\mathrm{GTC}>\mathrm{GTO}>\mathrm{DO}$ (Figure 1).

\subsection{Response surface methodology and optimization by Box and Behnken design}

\subsubsection{Response surface analysis and fitness to the model}

The influence of type of liquid lipid employed in each formulation, liquid lipid concentration, surfactant concentration and the amount of drug on CQAs taken as dependent variables viz. PS, PDI, ZP, \%EE and \%DL are graphically represented as contour plots (Figure 2. A \& B). The contour plots demonstrate the effect of the interaction between the liquid lipid concentration with different amounts of drug at medium level of Tween 80 (Figure 2.A) and interaction between the liquid lipid concentration and the Tween 80 concentration at a low level of drug for each type of liquid lipid on the selected CQAs (Figure 2.B).

The ANOVA of the regression model demonstrates that the model is highly significant as evident from the high $\mathrm{F}$ values ( $\mathrm{F}$ model for $\mathrm{PS}=4.91, \mathrm{PDI}=2.58, \mathrm{ZP}=2.59, \mathrm{EE}=4.79$ and $\mathrm{DL}=21.80$ ). Values of "Prob $>\mathrm{F}$ " less than 0.05 for all responses indicate model terms are significant (Table 3). A non-significant value of lack-of-fit shows that the model is suitable in 
fitting the data and predicting the response. It can be seen from predicted versus actual plots (Figure S1 and S2) that the experimentally measured values are well in line with the predicted values. Furthermore, adequate precision measures the signal to noise ratio. A ratio greater than 4 is desirable. The ratio of 8.98 (PS), 8.25 (PDI), 7.28 (ZP) 9.58 (EE) and 15.78 (DL) indicates an adequate signal and model can be used to navigate the design space. A quadratic model was implemented for all responses and polynomial equations were generated which explain the individual and interaction effects of independent factors on the dependent variables [25].

\subsubsection{Influence of investigated parameters on PS}

The PS was clearly affected by the attributes of liquid lipid incorporated in the NLCs. The more lipophilic oils, the two triglycerides GTO, GTC and the long chain fatty acid ester DO that were more miscible with the solid lipid resulted in lower PS as compared to the hydrophilic oils (PGC, PGMC and PGML). We hypothesize that lipophilic oils produced more stable NLCs with lower PS because the miscible oils likely promote a more mobile interface resulting in increased mobility of surfactant at the particle surface which allowed the surfactant molecules to move around the interface during the polymorphic transformation to stabilize uncovered hydrophobic surfaces. Higher PS upon the inclusion of Labrasol has been previously reported [26]. Further for each of the six RES-NLCs, the quadratic equations showing the effect of independent variables on PS are given below:

\section{For GTC}

$\mathrm{PS}=+103.33462+41.64833 \times \mathrm{X}_{1-} 68.36833 \times \mathrm{X}_{2-}-0.49571 \times \mathrm{X}_{3}-$

$88.83333 \times \mathrm{X}_{1} \times \mathrm{X}_{2}+0.12487 \times \mathrm{X}_{1} \times \mathrm{X}_{30} 0.083767 \times \mathrm{X}_{2} \times \mathrm{X}_{3}+23.18667 \times \mathrm{X}_{1}^{2}+52.46000 \times \mathrm{X}_{2}+2.32167$

$\mathrm{E}-003 \times \mathrm{X}_{3}^{2}$

Equation 4

\section{For DO}

$\mathrm{PS}=+122.61144+28.18333 \times \mathrm{X}_{1}-82.71333 \times \mathrm{X}_{2}-0.48106 \times \mathrm{X}_{3}-$

$88.83333 \times \mathrm{X}_{1} \times \mathrm{X}_{2}+0.12487 \times \mathrm{X}_{1} \times \mathrm{X}_{30} 0.083767 \times \mathrm{X}_{2} \times \mathrm{X}_{3}+23.18667 \times \mathrm{X}_{1}^{2}+52.46000 \times \mathrm{X}_{2}^{2}+2.32167$

$\mathrm{E}-003 \times \mathrm{X}_{3}^{2}$

Equation 5

\section{For PCG}

$\mathrm{PS}=+119.05913+32.47333 \times \mathrm{X}_{1}-79.84333 \times \mathrm{X}_{2}-0.51231 \times \mathrm{X}_{3}-88.83333 \times \mathrm{X}_{1} \times \mathrm{X}_{2}+0.12487 \times \mathrm{X}_{1} \times$ $\mathrm{X}_{3}-0.083767 \times \mathrm{X}_{2} \times \mathrm{X} 3+23.18667 \times \mathrm{X}_{1}^{2}+52.46000 \times \mathrm{X}_{2}^{2}+2.32167 \mathrm{E}-003 \times \mathrm{X}_{3}^{2}$ Equation 6 


\section{For PGMC}

$\mathrm{PS}_{=}+108.17029+82.83333 \times \mathrm{X}_{1}-50.11333 \times \mathrm{X}_{2}-0.63466 \times \mathrm{X} 3-88.83333 \times \mathrm{X}_{1} \times \mathrm{X}_{2}+0.12487 \times \mathrm{X}_{1}$ $\times \quad \mathrm{X}_{3}-0.083767 \times \mathrm{X}_{2} \times \mathrm{X} 3+23.18667 \times \mathrm{X}_{1}{ }^{2}+52.46000 \times \mathrm{X}_{2}{ }^{2}+2.32167 \mathrm{E}-003 \times \mathrm{X}_{3}{ }^{2}$

\section{Equation 7}

For GTO

$\mathrm{PS}=+121.03423+39.50833 \times \mathrm{X}_{1}-72.21333 \times \mathrm{X}_{2}-0.57738 \times \mathrm{X} 3-88.83333 \times \mathrm{X}_{1} \times \mathrm{X}_{2}+0.12487 \times \mathrm{X}_{1}$ $\times \quad \mathrm{X}_{3}-0.083767 \times \mathrm{X}_{2} \times \mathrm{X} 3+23.18667 \times \mathrm{X}_{1}{ }^{2}+52.46000 \times \mathrm{X}_{2}{ }^{2}+2.32167 \mathrm{E}-003 \times \mathrm{X}_{3}{ }^{2}$

\section{Equation 8}

\section{For PGML}

$\mathrm{PS}=+103.85404+71.22833 \times \mathrm{X}_{1}-70.10833 \times \mathrm{X}_{2}-0.52336 \times \mathrm{X}_{3}-88.83333 \times \mathrm{X}_{1} \times \mathrm{X}_{2}+0.12487 \times \mathrm{X}_{1} \times$ $\mathrm{X}_{3}-0.083767 \times \mathrm{X}_{2} \times \mathrm{X}_{3}+23.18667 \times \mathrm{X}_{1}^{2}+52.46000 \times \mathrm{X}_{2}^{2}+2.32167 \mathrm{E}-003 \times \mathrm{X}_{3}^{2}$ Equation 9

Positive sign before a factor in polynomial equations suggest a synergistic effect and signifies that the response increases with the factor, whereas a negative sign means the response and factors have reciprocal relation. The magnitudes of the coefficients indicate the degree of contribution of the factor to the response, the influence of six liquid lipids on PS was in the following order $\mathrm{PGMC}>\mathrm{PGML}>\mathrm{GTC}>\mathrm{GTO}>\mathrm{PCG}>\mathrm{DO}$ (equations 4-9). This is further vivid in perturbation plots (Figure 3.a) where steeper curvature for PGMC and PGML entail that these two liquid lipids had a higher impact on PS than other liquid lipids [27, 28]. Apart from type of liquid its concentration also has a significant effect on the PS of the NLCs which could be attributed to the swollen core of the nanoparticles loaded with liquid lipid. As the amount of the oil increases reduced emulsifying efficiency leads to higher surface tension and larger particles are formed. This corroborates with several previous reports [29, 30]. These observations are also elaborated in the contour plots (Figure 2. B)

$\mathrm{X}_{2}$ had a negative effect on the PS with highest impact when DO was used as liquid lipid and least when PGMC was employed in the NLC formulation. Higher concentration of surfactant results in reduction in the surface tension and production of particles with small sizes whereas lower concentration of surfactant would be insufficient to reduce the interfacial tension 
yielding larger size particle $[31,32]$. Furthermore, the surface activity of the surfactant offer stabilisation and prevent coalescence of nanoparticles [33]. $\mathrm{X}_{3}$ relatively had low impact on PS as evident from the small values of coefficient of $X_{3}$ in equations $4-9$. However, the interaction between $\left(\mathrm{X}_{1}-\mathrm{X}_{3}\right)$ had a positive effect on PS with liquid lipid concentration having more powerful impact than amount of drug added to NLCs. This effect is also evident in the contour plots (Figure 2. A).

\subsubsection{Influence of investigated parameters on PDI}

PDI is measure of the heterogeneity of sizes of particles in a mixture and an indicator of aggregation in the particles. The PDI values of $<0.250$ suggest that the nanoparticles exist in monodispersed distribution, with low variability and aggregation [34]. NLCs prepared using different liquid lipids varied in PDI values between 0.11 to 0.45 . As PS was impacted positively with increase in liquid lipid concentration we would expect an increase in PDI at higher concentrations of oil due to the increase in the agglomeration of the particles as a consequence of the Van der Waal attraction forces resulting in polydisperse formulations [23]. However, in exception to the two propylene glycol esters, PGMC and PGML which showed positive correlation, other liquid lipids either did not have much impact or resulted in formation of more homogeneous NLCs with increase in liquid lipid [35]. This is also evident from steep curvature of the RES-PGMC and RES-PGML in the perturbation plots signifying their higher influence on PDI (Figure 3.b). Of interest was the observation that at highest concentration of GTC, resulted in NLCs with low PDI (Figure 2.A \& B) [8]. The influences of liquid lipids on PDI was in the following decreasing order: PGML $>$ PGMC $>$ PGC $>$ GTC $>$ DO $>$ GTO $>$ PCG.

The effect of various parameters on the PDI is shown by the equations below:

\section{For GTC}

$\mathrm{PDI}=+0.35498-0.25950 \times \mathrm{X}_{1}-0.22717 \times \mathrm{X}_{2}+8.26667 \mathrm{E}-004 \times \mathrm{X}_{3}+0.18533 \times \mathrm{X}_{1} \times \mathrm{X}_{2}-1.53333 \mathrm{E}-$ $004 \times \mathrm{X}_{1} \times \mathrm{X}_{3}-5.43333 \mathrm{E}-004 \times \mathrm{X}_{2} \times \mathrm{X} 3+0.039000 \times \mathrm{X}_{1}^{2}+0.19033 \times \mathrm{X}_{2}^{2}-2.34167 \mathrm{E}-006 \mathrm{X}_{3}^{2}$

\section{Equation 10}

\section{For DO}

$\mathrm{Y}_{2}=+0.26509-0.12600 \times \mathrm{X}_{1}-0.31917 \times \mathrm{X}_{2}+1.36917 \mathrm{E}-003 \times \mathrm{X}_{3}+0.18533 \times \mathrm{X}_{1} \times \mathrm{X}_{2}-1.53333 \mathrm{E}-$

$004 \times \quad X_{1} \quad \times X_{3}-5.43333 E-004 \times X_{2} \quad \times X_{3}+0.039000 \times X_{1}^{2}+0.19033 \times \quad X_{2}^{2}-2.34167 E-006 \quad X_{3}^{2}$

\section{Equation 11}




\section{For PCG}

$\mathrm{PDI}=+0.061811-0.061500 \times \quad \mathrm{X}_{1}+0.010333 \times \quad \mathrm{X}_{2}+6.74167 \mathrm{E}-004 \times \quad \mathrm{X}_{3}+0.18533 \times \quad \mathrm{X}_{1} \times \mathrm{X}_{2}-$ $1.53333 \mathrm{E}-004 \times \mathrm{X}_{1} \times \mathrm{X}_{3}-5.43333 \mathrm{E}-004 \times \mathrm{X}_{2} \times \mathrm{X}_{3}+0.039000 \times \mathrm{X}_{1}^{2}+0.19033 \times \mathrm{X}_{2}^{2}-2.34167 \mathrm{E}-$

$006 \mathrm{X}_{3}^{2}$

Equation 12

\section{For PGMC}

$\mathrm{PDI}=+0.25382+1.16573 \mathrm{E}-015 \times \mathrm{X}_{1}-0.37767 \times \mathrm{X}_{2}+1.49167 \mathrm{E}-003 \times \mathrm{X}_{3}+0.18533 \times \mathrm{X}_{1} \times \mathrm{X}_{2}-$ 1.53333E-004 $\times \mathrm{X}_{1} \times \mathrm{X}_{3}-5.43333 \mathrm{E}-004 \times \mathrm{X}_{2} \times \mathrm{X}_{3}+0.039000 \times \mathrm{X}_{1}^{2}+0.19033 \times \mathrm{X}_{2}^{2}-2.34167 \mathrm{E}-$

$006 \mathrm{X}_{3}^{2}$

Equation 13

\section{For GTO}

$\mathrm{PDI}=+0.35697-0.12000 \times \mathrm{X}_{1}-0.42267 \times \mathrm{X}_{2}+1.29667 \mathrm{E}-003 \times \mathrm{X}_{3}+0.18533 \times \mathrm{X}_{1} \times \mathrm{X}_{2}-1.53333 \mathrm{E}-$ $004 \times \mathrm{X}_{1} \times \mathrm{X}_{3}-5.43333 \mathrm{E}-004 \times \mathrm{X}_{2} \times \mathrm{X}_{3}+0.039000 \times \mathrm{X}_{1}^{2}+0.19033 \times \mathrm{X}_{2}^{2}-2.34167 \mathrm{E}-006 \mathrm{X}_{3}^{2}$

\section{Equation 14}

\section{For PGML}

$\mathrm{PDI}=+0.27357+0.044000 \times \mathrm{X}_{1} 0.30917 \times \mathrm{X}_{2}+1.10417 \mathrm{E} 003 \times \mathrm{X}_{3}+0.18533 \times \mathrm{X}_{1} \times \mathrm{X}_{2} 1.53333 \mathrm{E} 004 \times$

$\mathrm{X}_{1} \times \mathrm{X}_{3}-5.43333 \mathrm{E}-004 \times \mathrm{X}_{2} \times \mathrm{X}_{3}+0.039000 \times \mathrm{X}_{1}^{2}+0.19033 \times \mathrm{X}_{2}^{2}-2.34167 \mathrm{E}-006 \mathrm{X}_{3}^{2}$

\section{Equation 15}

Increasing the amount of Tween 80 concentration led to better homogeneity in PS and reduced aggregation (equations 10-15). Higher amount of surfactant is known to cover the nanoparticles surface better thus preventing their coalescence [36]. If the thickness of the surfactant layer is high relative to NLC diameter, an efficient steric protection is assured, and the coalescence is prevented. The addition of higher amount of drug led to increased heterogeneity of NLC as demonstrated by the positive coefficient of this factor for all six type of liquid lipids (Figure

\section{A \& B).}

The interaction between the concentration of liquid lipid and the surfactant had a positive effect on the PDI, signifying their increase will cause increase in the PDI. Tween 80 concentration affected the NLCs prepared using GTC, PCG, PGMC and GTO, while DO and PGML 
containing NLCs did not show much effect on PDI. Furthermore, amount of drug only showed prominent perturbation effect with NLCs prepared using PCG on the PDI.

\subsubsection{Influence of investigated parameters on $\mathrm{ZP}$}

Generally, high ZP (negative or positive) is considered as a main contributing factor for the stability of the colloidal dispersions. Generally, ZP of $\pm 30 \mathrm{mV}$ is considered suitable to get a stable NLC dispersion because of electrical repulsion between the particles. ZP of NLCs varied in the range of -21.3 to $-39.9 \mathrm{mV}$. The negative charge can be associated with the presence of hydroxyl ions on the surface of the lipid nanostructures [37]. Moreover, polyphenolic drug or free fatty acids and partial glycerides present in the oils or phospholipids used as stabilizers would also contribute to the negative electrical charge of the NLCs [38].The quadratic polynomial equations below summarize the effect of three independent variables on the ZP of six liquid lipids NLCs:

\section{For GTC}

$\mathrm{ZP}=-54.56346+11.23333 \mathrm{x} \mathrm{X}_{1}+35.46667 \mathrm{xX}_{2}+0.062667 \mathrm{xX}_{3}+3.06667 \mathrm{x} \mathrm{X}_{1} \mathrm{x} \mathrm{X}_{2}-0.041333 \mathrm{x}$ $\mathrm{X}_{1} \quad \mathrm{x} \mathrm{X}_{3}-2.66667 \mathrm{E}-003 \mathrm{x} \quad \mathrm{X}_{2} \mathrm{x} \quad \mathrm{X}_{3}+0.26667 \times \mathrm{X}_{1}^{2}-17.20000 \mathrm{x} \quad \mathrm{X}_{2}^{2}-1.33333 \mathrm{E}-004 \mathrm{x} \quad \mathrm{X}_{3}^{2}$

\section{Equation 16}

\section{For DO}

$Z \mathrm{P}=-51.13269+8.93333 \mathrm{xX} 1+37.51667 \mathrm{x} \mathrm{X}_{2}+0.045417 \mathrm{x} \mathrm{X}_{3}+3.06667 \mathrm{x} \mathrm{X}_{1} \times \mathrm{X}_{2}-0.041333 \mathrm{x}$ $\mathrm{X}_{1} \quad \mathrm{x} \quad \mathrm{X}_{3}-2.66667 \mathrm{E}-003 \mathrm{XX}_{2} \mathrm{x} \mathrm{X}_{3}+0.26667 \mathrm{xX}_{1}^{2}-17.20000 \mathrm{xX}_{2}^{2}-1.33333 \mathrm{E}-004 \mathrm{xX}_{3}^{2}$

\section{Equation 17}

\section{For PCG}

$Z P=-38.50673-0.016667 \times X_{1}+28.76667 x X_{2}+0.046417 x_{3}+3.06667 x X_{1} \times X_{2}-0.041333 x$ $\mathrm{X}_{1} \quad \mathrm{xX}_{3}-2.66667 \mathrm{E}-003 \mathrm{xX}_{2} \mathrm{xX}_{3}+0.26667 \mathrm{x}_{1}^{2}-17.20000 \mathrm{xX}_{2}^{2}-1.33333 \mathrm{E}-004 \mathrm{xX}_{3}^{2}$

\section{Equation 18}

For PGMC

$Z P=-47.67308-7.26667 x X_{1}+38.31667 x X_{2}+0.050917 x X_{3}+3.06667 x X_{1} \times X_{2}-0.041333 x$ $\mathrm{X}_{1} \quad \mathrm{xX}_{3}-2.66667 \mathrm{E}-003 \mathrm{xX}_{2} \mathrm{XX}_{3}+0.26667 \mathrm{xX}_{1}^{2}-17.20000 \mathrm{xX}_{2}^{2}-1.33333 \mathrm{E}-004 \mathrm{xX}_{3}^{2}$

\section{Equation 19}




\section{For GTO}

$\mathrm{ZP}=-53.78462+2.13333 \mathrm{x} \mathrm{X} 1+38.76667 \mathrm{x} \mathrm{X}_{2}+0.075667 \mathrm{x} \mathrm{X}_{3}+3.06667 \mathrm{x} \quad \mathrm{X} 1 \mathrm{xX}_{2}-$
$0.041333 \mathrm{xX}_{1} \quad \mathrm{xX}_{3}-2.66667 \mathrm{E}-003 \mathrm{xX}_{2} \mathrm{XX}_{3}+0.26667 \mathrm{xX}_{1}^{2}-17.20000 \mathrm{xX}_{2}^{2}-1.33333 \mathrm{E}-004 \mathrm{xX}_{3}^{2}$

\section{Equation 20}

\section{For PGML}

$Z P=-42.83942-1.31667 x$ X1+26.66667x X2+0.057417x X3+3.06667x X1 x X2-0.041333x

$\mathrm{X}_{1} \quad \mathrm{x} \quad \mathrm{X}_{3}-2.66667 \mathrm{E}-003 \mathrm{XX}_{2} \mathrm{XX} 3+0.26667 \mathrm{x}_{1}{ }^{2}-17.20000 \mathrm{xX}_{2}^{2}-1.33333 \mathrm{E}-004 \mathrm{XX}_{3}^{2}$

\section{Equation 21}

The hydrophilic liquid lipids played a more prominent role contributing to the increase in the negative surface charge (equations 16-21), due to the adsorption of free hydroxyl ions present in these liquid lipids. Increase in their concentration resulted in higher ZP and improved stability with their influence in order PGMC $>$ PCG $>$ PGML. While increasing the concentration of lipophilic liquid lipids which do not possess any free hydroxyl groups resulted in lowered ZP and decreased NLC stability with their influence in order of GTC $>$ DO $>$ GTO. Tween 80 being a non-ionic surfactant with an HLB value of 15 being adsorbed on the surface of particles causes reduction in the net charge at the particle surface, resulting in negatively charged NLCs [39]. Surfactant concentration showed higher effect on zeta potential with PGMC, GTO, GTC and DO containing NLCs (Figure 3. c). Increasing the RES content resulted in increased negative charge on NLCs as would be expected due to polyphenolic structure of the drug. However, the overall influence of drug amount was less on ZP as concluded from the lower coefficient values of $\mathrm{X}_{3}$ and 2-D contour plots (Figure 2. A \& B). The interaction between the factors $\left(\mathrm{X}_{1}-\mathrm{X}_{2}\right.$ and $\left.\mathrm{X}_{1}-\mathrm{X}_{3}\right)$ for all liquid lipid type had positive effect on the ZP. Interestingly, the interaction between $\mathrm{X}_{2}-\mathrm{X}_{3}$ had a negative effect on the ZP even though both factors individually demonstrated a positive effect on the response.

\subsubsection{Influence of investigated parameters on $\mathbf{E E}$}

EE of RES in all the NLC formulations with six liquid lipids were observed to be in range of 91.9 to $99.5 \%$ with NLCs with DO showing the highest EE. The influence of liquid lipid on EE was in the order DO $>$ PCG $>$ GTC $>$ PGMC $>$ GTO $>$ PGML. High EE $>90 \%$ for all NLCs could be attributed to crystal imperfections that provide more space for drug incorporation [32]. The effect on EE can be explained by quadratic equations below: 


\section{For GTC}

$\mathrm{EE}=+90.67318-$

$3.05300 \times \mathrm{X}_{1} 7.24967 \times \mathrm{X}_{2}+0.092645 \times \mathrm{X}_{3}+0.29733 \times \mathrm{X}_{1} \times \mathrm{X}_{2}+0.020640 \times \mathrm{X}_{1} \times \mathrm{X}_{3}+0.018607 \times \mathrm{X}_{2} \times \mathrm{X}$ $3-1.38400 \times \mathrm{X}_{1}^{2}+3.78800 \times \mathrm{X}_{2}^{2}-2.81267 \mathrm{E}-004 \times \mathrm{X}_{3}^{2}$

Equation 22

\section{For DO}

$\mathrm{EE}=+97.14014-$

$5.71900 \times X_{1} 9.28967 \times X_{2}+0.069875 \times X_{3}+0.29733 \times X_{1} \times X_{2}+0.020640 \times X_{1} \times X_{3}+0.018607 \times X_{2} \times X$ ${ }_{3}-1.38400 \times \mathrm{X}_{1}^{2}+3.78800 \times \mathrm{X}_{2}^{2}-2.81267 \mathrm{E}-004 \times \mathrm{X}_{3}^{2}$

Equation 23

\section{For PCG}

$\mathrm{EE}=+88.62314-$

$3.16100 \times \mathrm{X}_{1} 9.36067 \times \mathrm{X}_{2}+0.11123 \times \mathrm{X}_{3}+0.29733 \times \mathrm{X}_{1} \times \mathrm{X}_{2}+0.020640 \times \mathrm{X}_{1} \times \mathrm{X}_{3}+0.018607 \times \mathrm{X}_{2} \times \mathrm{X}_{3}$

$-1.38400 \times \mathrm{X}_{1}^{2}+3.78800 \times \mathrm{X}_{2}^{2}-2.81267 \mathrm{E}-004 \times \mathrm{X}_{3}^{2}$

Equation 24

\section{For PGMC}

$\mathrm{EE}=+91.63166-2.22400 \times \mathrm{X}_{1-}$

$7.65667 \times \mathrm{X}_{2}+0.083855 \times \mathrm{X}_{3}+0.29733 \times \mathrm{X}_{1} \times \mathrm{X}_{2}+0.020640 \times \mathrm{X}_{1} \times \mathrm{X}_{3}+0.018607 \times \mathrm{X}_{2} \times \mathrm{X}_{3}-$

$1.38400 \times \mathrm{X}_{1}^{2}+3.78800 \times \mathrm{X}_{2}^{2}-2.81267 \mathrm{E}-004 \times \mathrm{X}_{3}^{2}$

Equation 25

\section{For GTO}

$\mathrm{EE}=+89.21141-$

$1.61100 \times \mathrm{X}_{1} 9.08167 \times \mathrm{X}_{2}+0.10384 \times \mathrm{X}_{3}+0.29733 \times \mathrm{X}_{1} \times \mathrm{X}_{2}+0.020640 \times \mathrm{X}_{1} \times \mathrm{X}_{3}+0.018607 \times \mathrm{X}_{2} \times \mathrm{X}_{3}$

$-1.38400 \times \mathrm{X}_{1}^{2}+3.78800 \times \mathrm{X}_{2}^{2}-2.81267 \mathrm{E}-004 \times \mathrm{X}_{3}^{2}$

Equation 26

\section{For PGML}

$\mathrm{EE}=+93.11872-1.21300 \times \mathrm{X}_{1-}$

$9.13767 \times \mathrm{X}_{2}+0.079765 \times \mathrm{X}_{3}+0.29733 \times \mathrm{X}_{1} \times \mathrm{X}_{2}+0.020640 \times \mathrm{X}_{1} \times \mathrm{X}_{3}+0.018607 \times \mathrm{X}_{2} \times \mathrm{X}_{3}-$ $1.38400 \times \mathrm{X}_{1}^{2}+3.78800 \times \mathrm{X}_{2}^{2}-2.81267 \mathrm{E}-004 \times \mathrm{X}_{3}^{2}$

Equation 27 
The amount of drug added in the NLCs showed most significant and a positive effect on the EE (equations 22-27). This is attributed to more drug being available for the entrapment in the internal phase which is also illustrated by the steep curvature for this factor in perturbation plots (Figure 3.d). Increasing the concentration of surfactant lead to lowered EE as higher amount of deposition of surfactant on the interface between the water and the lipid phase reduced interfacial tension which would increase the shear pressure generated during the homogenization process that would result in the formation of small emulsion droplet and thus decreasing the EE [40]. The interaction between $\left(\mathrm{X}_{1}-\mathrm{X}_{2}, \mathrm{X}_{1} \mathrm{X}_{3}\right.$ and $\left.\mathrm{X}_{2} \mathrm{X}_{3}\right)$ for all liquid lipids had a positive effect on the EE even though $\mathrm{X}_{1}$ and $\mathrm{X}_{2}$ showed a negative effect on the EE, but the combination with the third factor $X_{3}$ made the interaction positive (Figure 2. A \& B). This can be used to predict interactions and determine the optimised formulation with desirable value of the response variable.

\subsubsection{Influence of investigated parameters on $D L$}

Drug loading is the major challenge in the formulation of nanoparticles, especially for poorly water-soluble drugs. Because of the disturbance in of the solid lipid crystal order achieved by incorporation of liquid lipid, higher drug incorporation due to more space to accommodate drug molecules can be obtained in NLCs as compared to SLNs [41]. Compared with the reported solubility of RES in water $(300 \mathrm{mg} / 100 \mathrm{~mL})$ [42] the solubility of RES in lipid matrices consisting of trimyristin and different oils was improved by at least 33.3 folds. The DL of various formulated NLCs ranged between 2.93 to $7.55 \%$, which indicates that the response was extremely sensitive toward the studied factors. The physicochemical properties of liquid lipid play a critical role on the DL in the NLC formulations. Labrasol, a PEGylated lipid with high HLB and solubility for the drug, resulted in higher DL as compared to GTO, DO and GTC which had lower solubility for the drug and lower HLB values. A substantially higher DL was achieved with RES-NLC-PCG as compared to a previous report on RES lipid nanoparticles [43] which was followed by the two polyethylene glycol esters PGML and PGMC showing comparable drug loading.

Increasing the amount of liquid lipids resulted in RES-NLCs with higher DL [29], as demonstrated by positive coefficients of $\mathrm{X}_{1}$, the influence of liquid lipid concentration was in the following order $\mathrm{PGMC}>\mathrm{PCG}>\mathrm{GTO}>\mathrm{PGML}>\mathrm{GTC}>\mathrm{DO}$. This is attributed to the increase in the auxiliary spaces in the lipid matrix due to distortion of its crystalline structure by the liquid lipids and their solubilizing effect on RES [32]. Apart from the nature of liquid lipid, the 
surfactant concentration and the amount of drug also impact the RES loading in the NLCs, which is demonstrated by the following quadratic equations.

\section{For GTC}

$\mathrm{DL}=-3.36946+3.31783 \times \mathrm{X}_{1}+7.79100 \times \mathrm{X}_{2}+0.039990 \times \mathrm{X}_{3}-0.12600 \times \mathrm{X}_{1} \times \mathrm{X}_{2}-3.64000 \mathrm{E}-003 \times$ $\mathrm{X}_{1} \quad \times \quad \mathrm{X}_{3}+5.67000 \mathrm{E}-003 \times \mathrm{X}_{2} \times \mathrm{X}_{3}-2.53333 \times \mathrm{X}_{1}^{2}-6.11133 \times \mathrm{X}_{2}^{2}-4.13667 \mathrm{E}-005 \times \mathrm{X}_{3}^{2}$

\section{Equation 28}

For DO

$\mathrm{DL}=-5.18163+2.23283 \times \mathrm{X}_{1}+10.78100 \times \mathrm{X}_{2}+0.039805 \times \mathrm{X}_{3}-0.12600 \times \mathrm{X}_{1} \times \mathrm{X}_{2}-3.64000 \mathrm{E}-$ $003 \times \mathrm{X}_{1} \times \mathrm{X}_{3}+5.67000 \mathrm{E}-003 \times \mathrm{X}_{2} \times \mathrm{X}_{3}-2.53333 \times \mathrm{X}_{1}^{2}-6.11133 \times \mathrm{X}_{2}^{2}-4.13667 \mathrm{E}-005 \times \mathrm{X}_{3}^{2}$

\section{Equation 29}

\section{For PCG}

$\mathrm{DL}=-5.03404+4.31733 \times \mathrm{X}_{1}+9.04800 \times \mathrm{X}_{2}+0.042647 \times \mathrm{X}_{3}-0.12600 \times \mathrm{X}_{1} \times \mathrm{X}_{2}-3.64000 \mathrm{E}-003 \times$ $\mathrm{X}_{1} \quad \times \quad \mathrm{X}_{3}+5.67000 \mathrm{E}-003 \times \mathrm{X}_{2} \times \mathrm{X}_{3}-2.53333 \times \mathrm{X}_{1}^{2}-6.11133 \times \mathrm{X}_{2}^{2}-4.13667 \mathrm{E}-005 \times \mathrm{X}_{3}^{2}$

\section{Equation 30}

\section{For PGMC}

$\mathrm{DL}=-4.81689+4.39033 \times \mathrm{X}_{1}+8.67450 \times \mathrm{X}_{2}+0.042885 \times \mathrm{X}_{3}-0.12600 \times \mathrm{X}_{1} \times \mathrm{X}_{2}-3.64000 \mathrm{E}-003 \times$ $\mathrm{X}_{1} \quad \times \quad \mathrm{X}_{3}+5.67000 \mathrm{E}-003 \times \mathrm{X}_{2} \times \mathrm{X}_{3}-2.53333 \times \mathrm{X}_{1}{ }^{2}-6.11133 \times \mathrm{X}_{2}{ }^{2}-4.13667 \mathrm{E}-005 \times \mathrm{X}_{3}{ }^{2}$

\section{Equation 31}

\section{For GTO}

$D L=-5.74321+3.74583 \times X_{1}+9.04100 \times X_{2}+0.049065 \times X_{3}-0.12600 \times X_{1} \times X_{2}-3.64000 E-003 \times$ $\mathrm{X}_{1} \quad \times \quad \mathrm{X}_{3}+5.67000 \mathrm{E}-003 \times \mathrm{X}_{2} \times \mathrm{X}_{3}-2.53333 \times \mathrm{X}_{1}^{2}-6.11133 \times \mathrm{X}_{2}^{2}-4.13667 \mathrm{E}-005 \times \mathrm{X}_{3}^{2}$

\section{Equation 32}




\section{For PGML}

$$
\begin{aligned}
& \mathrm{DL}=-4.78191+3.72333 \times \mathrm{X}_{1}+9.12850 \times \mathrm{X}_{2}+0.041120 \times \mathrm{X}_{3}-0.12600 \times \mathrm{X}_{1} \times \mathrm{X}_{2}-3.64000 \mathrm{E}-003 \times \\
& \mathrm{X}_{1} \times \quad \mathrm{X}_{3}+5.67000 \mathrm{E}-003 \times \mathrm{X}_{2} \times \mathrm{X}_{3}-2.53333 \times \mathrm{X}_{1}{ }^{2}-6.11133 \times \mathrm{X}_{2}{ }^{2}-4.13667 \mathrm{E}-005 \times \mathrm{X}_{3}{ }^{2}
\end{aligned}
$$

\section{Equation 33}

It is evident that the Tween 80 concentration exerted a positive effect with enhanced entrapped drug as apparent from the positive values of the coefficient $\left(\mathrm{X}_{2}\right)$ in equations $28-33 . \mathrm{X}_{3}$ had a positive effect on the DL with higher drug being entrapped inside the nanoparticles when more drug was added [32, 44]. This is also evident from the perturbation plots (Figure 3.e). Interestingly, the interaction between factors $(\mathrm{X} 1-\mathrm{X} 2)$ and $(\mathrm{X} 1-\mathrm{X} 3)$ for all the types of liquid lipids had a negative effect on the $\% \mathrm{DL}$, even though all three factors had a positive effect on this response when taken individually (Figure 2.A \& B). On the other hand, the interaction between $\left(\mathrm{X}_{2}-\mathrm{X}_{3}\right)$ demonstrated a positive effect, which signifies that any increase in each of these factors will lead to the increase in the DL.

\subsubsection{Design Space}

Design space is referred as the multidimensional combination and interaction of input variables and process parameters that have been demonstrated to provide assurance of quality [45] and was obtained by overlaying the critical response contours with overlay plot (Figure 4). The yellow coloured regions describe the design space with suitable response values and grey regions illustrate where response values did not meet the quality product attributes. Based on the overlay plot and desirability function (Figure S3) criteria the optimized formulations can be selected from the design space. The selection of the optimized NLCs was based upon accomplishing the minimum particle size (50 to $100 \mathrm{~nm}$ ), lowest PDI value (0.11 to 0.30), formation of stable particles (ZP) (-37 to $-21 \mathrm{mV})$ maximum value of EE (94 to $99.54 \%)$ and maximum DL (3.00 to $7.55 \%$ ) (Table 4). This model can be used to navigate the design space.

\subsection{Differential Scanning Calorimetry (DSC)}

DSC is a well-established technique that offers a close look of the melting and crystallization behaviour of crystalline material like lipid nanoparticles to characterize the physical and chemical changes in either their enthalpy or the heat capacity of the lipid [46].

DSC thermogram of trimyristin displayed a sharp endothermic peak at $58.32^{\circ} \mathrm{C}$ (Figure 5.A). The addition of all six liquid lipids to trimyristin caused a depression in its melting point (MP) [47], however, the degree of MP depression was affected by the type and concentration of the 
liquid lipid, whereby the maximum reduction of MP occurred at the highest concentration of each of the oil. The reduction of onset temperature on addition of each of the liquid lipids to trimyristin followed the subsequent order $\mathrm{PGMC}<\mathrm{PGML}<\mathrm{DO}<\mathrm{GTC}<\mathrm{GTO}<\mathrm{PCG}$. The difference between the melting and the peak onset temperatures is the point at which the melting of the lipid occurs after addition of the liquid lipid, and the greater difference infers higher disordered state of the crystals. The lower concentrations of liquid lipids resulted in a lower difference (around 2 degrees), whereas when concentration of the oils was increased to $0.75 \%$, major differences between the onset and the melting temperature was observed indicating greater disorder in the structure (Figure S4.a) [48]. The highest difference between melting and peak onset temperature was demonstrated by PGMC (6 degrees) clearly causing maximum distortion in the crystal lattice of trimyristin. This is in corroboration with a previous report showing a linear correlation between the concentration of oil and the melting point depression indicating complete incorporation of liquid lipid [49]. The data also demonstrated reduction in the melting enthalpy of the solid lipid on addition of liquid lipids with maximum reduction being revealed by GTO (Figure S4.b). Trimyristin with an enthalpy of $134.62 \mathrm{~J} / \mathrm{g}$ in the bulk, its value was reduced to $94.39,95.37,102.43,106.34,107.78$ and $110.93 \mathrm{~J} / \mathrm{g}$ on addition of GTO, DO, PCG, PGMC, PGML and GTC, respectively. For the less ordered crystal state, the melting of the solid lipid required less energy to overcome the lattice force.

DSC analysis was also performed to study the effect of the incorporation of different liquid lipids on the crystallinity of RES and its melting behaviour (Figure 5.B). Addition of liquid lipids to RES showed a concentration dependent depression in its MP with a linear correlation. RES demonstrated a sharp endothermic peak at $267.49^{\circ} \mathrm{C}$ which was distinctly shifted to 4.21 , 105.41, 218.76, 237.52, 247.19 and $258.29^{\circ} \mathrm{C}$ in presence of PGMC, PCG, PGML, DO, GTC and GTO, respectively. We can observe that nature of liquid lipid had huge impact on depression of MP of RES, with PGMC causing great disturbance and distortion of the crystal lattice of RES. At the highest concentration $(0.75 \%)$ of PGMC, the drug was completely dissolved and the thermogram did not therefore reveal any endothermic peak of drug indicating it to be in amorphous form, though at lower concentrations of PGMC the crystallinity of drug was still maintained. In comparison to other liquid lipids, PCG showed the lowest onset temperature and lowest melting temperature (Figure S5.a). It also elicited greatest difference between the melting and the onset temperatures indicating greatest distortion of the drug crystal lattice by $\mathrm{PCG}$. This was followed by $\mathrm{PGML}>\mathrm{DO}>\mathrm{GTC}>\mathrm{GTO}$ respectively, which also lowered the MP and onset temperatures of drug in that order. Generally, amorphous materials 
possess higher saturation solubility than crystalline materials; however, amorphous drugs in metastable state can spontaneously recrystallize and lead to a decreasing bioavailability during the process of storage. To avoid this, the ideally produced nanoparticle system should be crystalline $[50,51]$. In this context, DO and GTO showed well-defined peak of RES at all oil concentrations indicating maintenance of crystalline structure of the drug even at high concentrations of liquid lipid [51] Data also depicted a significant decrease in the melting enthalpy of RES with a maximum reduction observed when the concentration of liquid lipids was at highest level (Figure S5.b) resulting in a less ordered crystalline state where the melting of the drug required less energy to overcome lattice force [52]. Further the use of high melting point triglyceride, trimyristin may reduce the mobility of the drug molecules within the lipid core and thus reduce the drug expulsion upon storage [53, 54].

All drug loaded and blank NLCs showed a single endothermic peak in the range of $103-116^{\circ} \mathrm{C}$ (Figure 5. C). The crystallization of lipid in its bulk state occurs differently from its emulsified state. Usually a shift of melting transition to a lower temperature is expected in a NLCs than the bulk lipid attributed to its small size. However, trimyristin NLCs showed endothermic peak at higher temperatures as compared to bulk lipid $\left(58.32^{\circ} \mathrm{C}\right)$. This could be due to faster polymorphic transitions of lipid crystals in NLCs due to their small size than in bulk [54]. Further emulsifiers present in NLCs are known to promote $\beta$ crystallization which is more stable and higher melting polymorph [55]. Further the chemical nature of liquid lipid influenced the melting behavior of the solid lipid in the NLCs. Addition of the two hydrophobic long chain oleates DO and GTO exhibited a shift to higher melting points, 116 and $111^{\circ} \mathrm{C}$ respectively, than other more hydrophilic liquid lipids $\left(103-104^{\circ} \mathrm{C}\right)$. The shift in endothermic peak along with the absence of characteristic RES endothermic peak at $267.14^{\circ} \mathrm{C}$ suggested interaction of RES with lipid components and indicated that RES entrapped in lipids was in amorphous state or dispersed molecularly in the NLCs [56].

\subsection{Storage stability of RES-NLCs}

PS and PDI of nanoparticles are two important criteria as these factors play a critical role in cellular uptake, drug release rate, bioavailability and bio-distribution to various tissues and the stability of the formulated products [57]. NLCs have the advantage of improved DL and controlled drug release and have demonstrated good stability in terms of PS and prevention of drug leakage from the nanoparticles during storage [58]. It would be of significant importance 
to examine if the nature of liquid lipid determines the stability of the NLCs. The six optimised NLCs containing six different liquid lipids (Table 4) were selected in order to study their stability and effect of long-term storage at two different temperatures $4^{\circ} \mathrm{C}$ and $20^{\circ} \mathrm{C}$ on the PS, PDI, ZP, EE and DL.

The stability studies clearly demonstrated that the type of liquid lipid greatly influenced the CQAs of the NLCs. The mean PS at $4^{\circ} \mathrm{C}$ of all NLCs was below $100 \mathrm{~nm}$ (Figure 6. a) except the one containing PGML which showed increased PS of $209.8 \pm 11.6 \mathrm{~nm}$ at the end of one month (Figure 7.A.b). The NLCs containing the PEGylated liquid lipids, Labrasol and the two propylene glycol esters, PGML and PGMC showed increase in PS to $<200 \mathrm{~nm}$ at the end of 3 months with some aggregation as demonstrated by peaks in the micron region in particle size distribution curves (Figure 7A.c) [59]. The particles grew in size with time with higher degree of aggregation at the end of six months with mean PS of above $200 \mathrm{~nm}$ for PCG, PGMC and PGML containing NLCs, while the NLCs formulated with two triglycerides GTO and GTC and the fatty acid ester DO maintained the mean PS below $200 \mathrm{~nm}$ even at the end of six months (Figure 7A.d). This could be explained due to probable migration of these liquid lipids towards the interface as compared to the more nonpolar oils which remained mixed with solid lipid trimyristin due their structural similarity. Previous studies have shown medium chain triglycerides to have stabilising effect on the long chain triglyceride emulsion when prepared as mixed emulsion [60]. Furthermore, crystallisation of drug at low temperature can contribute to increase in PS. The NLCs stored at $20^{\circ} \mathrm{C}$ showed better stability with mean PS well maintained below $100 \mathrm{~nm}$ up to three months (Figure 6.a). However, beyond three months, NLCs containing PGMC and PGML showed a significant increase in the PS which increased above $100 \mathrm{~nm}$ at the end of six months (Figure 7A.f \& g).

The PDI values for all formulations stored at $4{ }^{\circ} \mathrm{C}$ showed an increase after one month except for GTC containing NLCs, which maintained low PDI $(0.27 \pm 0.03)$ (Figure 6.b). After three months of storage, NLCs with PCG and PGMC were highly polydisperse with distinct aggregation. Six months stability data showed an overall increase of PDI above 0.3 for all the NLCs. Meanwhile, NLCs stored at $20^{\circ} \mathrm{C}$ revealed no pronounced changes in the PDI. This could probably be due to better maintenance of lipid structure and drug remaining entrapped within it at ambient temperature, whereas storage at lower temperature lead to crystallization of the drug and eventually increase in the PS of NLCs [61, 62]. The increase in PS on storage is attributed to a number of mechanisms including coalescence, flocculation and Ostwald 
ripening. Flocculation and coalescence lead usually to a wide particle size distribution with a high PDI [37].

Coalescence phenomenon is described by the equation 34 :

$1 / \mathrm{r}^{2}=/ \mathrm{r}^{2}{ }_{0}-8 \pi / 3 \omega \mathrm{t}$

Equation 34

Where $r$ is the average of radius of NLCs, $r_{0}$ is the radius value at $t=0$ and $\omega$ is the frequency of rupture per unit of surface of the film [62]. Another mechanism for instability is Ostwald ripening phenomenon, which describes the solubility of smaller particles due to high surface energy within the dispersion medium and redeposit onto larger particles [61]. The Ostwald ripening rate can be evaluated by applying the principal, which predicts a linear relationship between the cube of particle radius, $r^{3}$, and time $t, \omega$ being the slope of plots (equation 35) [62].

$\omega=\mathrm{dr}^{3} / \mathrm{dt}=8 / 9\left[\mathrm{C}_{\infty} \gamma\left(\mathrm{MD} / \rho^{2} \mathrm{RT}\right)\right] \quad$ Equation 35

The main physicochemical mechanism contributing to particle growth of the NLCs is due Ostwald ripening more than coalescence and flocculation (Figure 8.a $\boldsymbol{\&}$ b). The plots of change of PS (radius, $\mathrm{r}^{3}$ ) with time for NLCs with PGMC, DO, PGML and PCG exhibited Oswald ripening as phenomenon for particle growth as evident from $\mathrm{r}^{2}$ values (Table S1) This could be associated to higher solubility of these oils in aqueous phase.However, NLCs with GTO and GTC showed coalescence to be the main mechanism of particle growth as evident from linear plots of $1 / \mathrm{r}^{2}$ vs. $t$ (Table S1). When PGMC was used as liquid lipid in NLCs, PS grew exponentially with time with large particles visible at the end of six months and the mechanism for particle grow might be attributed to flocculation (Figure 8.a \& b).

ZP reflects the electric charge on the particle surface and is a key indicator for predicting the long-term physical stability of colloidal dispersion system [63]. It was interesting to note that though ZP of the fresh NLCs prepared with six different liquid lipids were not significantly different from each other (Figure 7 B. a), time dependent change in ZP was strongly influenced by the type of liquid lipid carrier (Figure 6.c). After storage at $4^{\circ} \mathrm{C}$, the ZP of the NLCs prepared with the triglycerides, GTO and GTC showed no evident reduction in the ZP value in the first month (Figure 7 B. b). This validates the PS and PDI results substantiating that the triglycerides with low melting point can impart stability to the NLC dispersions. Also DO containing NLCs did not show a reduction of ZP value in the first month while propylene glycol esters PGML and PGMC containing NLCs revealed sharp decline in ZP $(-11.1 \mathrm{mV})$ demonstrating their poor colloidal stability. After three months, a considerable reduction in the 
ZP value (Figure 7 B. c) for all NLCs were observed which further deteriorated at the end of six-months on storage at $4^{\circ} \mathrm{C}$ (Figure 7B. d). The generation of long $\beta$-crystals of trimyristin during storage with their re-orientation and differently charged crystal side on the surface could be responsible for the variation in the ZP [64]. NLCs with GTO, PCG and GTC at $20^{\circ} \mathrm{C}$ showed good stability for the first three months (Figure 7B.e \&f). In addition, NLCs with the triglycerides, GTO and GTC showed minimal change of ZP at the end of six months, while all other formulations had a significant reduction in their ZP values over the period of six months (Figure 7B.g). Thus, the stability of NLCs in terms of PS and ZP was much better when stored at $20^{\circ} \mathrm{C}$ rather than $4^{\circ} \mathrm{C}$.

With NLCs stored at $4^{\circ} \mathrm{C}$, the entrapped drug remained stable with no change in the total drug content after one month (Figure 6.d). However, only NLCs containing GTO showed good stability upon storage at $25^{\circ} \mathrm{C}$ for the first month (Figure 6.d). After 3 months, all formulations showed a significant decrease in drug content regardless of their storage conditions, potentially suggesting degradation of the drug with time. RES being prone to thermal degradation and at $\mathrm{pH}$ above 6.8, could be the cause for lowered drug content in the formulation on long term storage [65].

The EE depends on the concentration and the type of lipid mixture utilized in formulating the NLCs. Incorporation of RES resulted in a high EE, because of the lipophilic nature of the drug. While all NLCs stored at both $4^{\circ} \mathrm{C}$ and $20^{\circ} \mathrm{C}$ showed significant difference in EE from the initial observed values (Figure 6.e) however still the values were reasonably high above 90\% EE. The high EE despite of low drug content is probably due to minimum drug leakage from the nanoparticles in spite of the degradation of the drug. Overall, NLCs made from GTO as a liquid lipid showed the best stability as compared to other NLCs over the mentioned period of time, maintaining average particle size less than $100 \mathrm{~nm}$, monodisperse formulation and requisite $\mathrm{EE}$ and $\mathrm{DL}$ when stored at $20^{\circ} \mathrm{C}$. This is in confirmation with DSC results which showed that crystalline structure of the drug was maintained in presence of GTO thus avoiding any amorphous metastable state or spontaneous recrystallization which contribute to instability of NLCs.

\section{Conclusion}

Past decade has seen dramatic escalation in the NLC based formulations reported in the literature, however there is no trend or direction in the selection of solid or liquid lipids and random combinations of lipids have been employed for preparation of NLCs. The present study 
clearly demonstrated the impact the different liquid lipids with varied chemical compositions, molecular structures and HLB have on the critical quality parameters, thermal behaviour and stability of NLCs. The more lipophilic oils, the two triglycerides GTO, GTC and the long chain fatty acid ester DO yield NLCs with lower PS as compared to the hydrophilic oils (PGC, PGMC and PGML). Though increasing the concentration of liquid lipid increased the PS of NLCs, however the impact on its polydispersity varied with the type of lipids with propylene glycol esters, PGMC and PGML showing positive correlation. The DL was dependent on the solubilizing effect of liquid lipid and its influence on the crystallinity of the drug and solid lipid. The varied liquid lipids not only impacted the degree of MP depression of solid lipid but also the crystallinity of drug and finally the stability of NLCs. The particle growth whether Oswald's ripening or coalescence or flocculation was also affected by the attributes of the liquid lipid incorporated in the NLCs. Finally, tuneable NLCs with RES as model drug could be manufactured with particles size $(<100 \mathrm{~nm})$, particle size distribution $(<0.3)$, with negatively charged surface (-24 mV), high EE (91-99\%) and DL (2-7\%) within the established design space, which may serve as useful carrier for the oral delivery of the bioactive. Further the study informs the rational design and provides vital insight on selection of liquid lipids and its significance for future development of NLCs for various drug delivery applications.

\section{Conflict of interest}

This research work is original, and all authors report no conflicts of interest.

\section{Author contributions}

The manuscript was written through contributions of all authors. All authors have given approval to the final version of the manuscript.

\section{Acknowledgement}

The authors are grateful to Lipoid GmbH, Germany; Gattefosse (UK) Limited; BASF and Cremer (Oleo), UK for providing us with gift samples of the excipients used in the formulations.

\section{References}

1. Talegaonkar, S. and A. Bhattacharyya, Potential of lipid nanoparticles (SLNS and NLCS) in enhancing oral bioavailability of drugs with poor intestinal permeability. AAPS PharmSciTech, 2019. 20(3): p. 121. 
2. Harisa, G.I. and M.M. Badran, Simvastatin nanolipid carriers decreased hypercholesterolemia induced cholesterol inclusion and phosphatidylserine exposure on human erythrocytes. Journal of Molecular Liquids, 2015. 208: p. 202-210.

3. Pinto, F., et al., Optimization of nanostructured lipid carriers loaded with retinoids by central composite design. Journal of Molecular Liquids, 2019. 293: p. 111468.

4. Cirri, M., et al., Design, characterization and in vivo evaluation of nanostructured lipid carriers (NLC) as a new drug delivery system for hydrochlorothiazide oral administration in pediatric therapy. Drug delivery, 2018. 25(1): p. 1910-1921.

5. Kawish, S.M., et al., Development of nabumetone loaded lipid nano-scaffold for the effective oral delivery; optimization, characterization, drug release and pharmacodynamic study. Journal of Molecular Liquids, 2017. 231: p 514-522..

6. $\quad$ Niu, Z., et al., Lipid-based nanocarriers for oral peptide delivery. Advanced drug delivery reviews, 2016. 106: p. 337-354.

7. Khosa, A., S. Reddi, and R.N. Saha, Nanostructured lipid carriers for site-specific drug delivery. Biomedicine and Pharmacotherapy, 2018. 103: p. 598-613.

8. Yang, Y., et al., The effect of oil type on the aggregation stability of nanostructured lipid carriers. Journal of Colloid and Interface Science, 2014. 418: p. 261-272.

9. Beloqui, A., et al., Nanostructured lipid carriers: Promising drug delivery systems for future clinics. Nanomedicine, 2016. 12(1): p. 143-61.

10. Ribeiro, A.P.B., et al., Crystallization modifiers in lipid systems. Journal of food science and technology, 2015. 52(7): p. 3925-3946.

11. Houacine, C. and K.K. Singh, 10 Nano Resveratrol: A Promising Future Nanonutraceutical. NanoNutraceuticals, Eds. Bhupinder Singh, Minna Hakkarainen, K.K.Singh, CRC Press, Taylor \& Francis Group, 2018.

12. Ha, E.-S., et al., Solubility of trans-resveratrol in transcutol HP+ water mixtures at different temperatures and its application to fabrication of nanosuspensions. Journal of Molecular Liquids, 2019. 281: p. 344-351.

13. Vankayala, J.S., et al., Surfactants and fatty alcohol based novel nanovesicles for resveratrol: Process optimization, characterization and evaluation of functional properties in $R A W 264.7$ macrophage cells. Journal of Molecular Liquids, 2018. 261: p. 387-396.

14. Abul Kalam, M., et al., Part I: Development and optimization of solid-lipid nanoparticles using Box-Behnken statistical design for ocular delivery of gatifloxacin. Journal Biomed Material Research A, 2013. 101(6): p. 1813-27.

15. Waghule, T., et al., Voriconazole loaded nanostructured lipid carriers based topical delivery system: QbD based designing, characterization, in-vitro and ex-vivo evaluation. Journal of Drug Delivery Science and Technology, 2019. 52: p. 303-315.

16. Joshi, M. and V. Patravale, Formulation and Evaluation of Nanostructured Lipid Carrier (NLC)-based Gel of Valdecoxib. Drug Development and Industrial Pharmacy, 2006. 32(8): p. 911-918.

17. Ratola, N., J. Faria, and A. Alves, Analysis and Quantification of trans-Resveratrol in Wines from Alentejo Region (Portugal). Food Technology and Biotechnology, 2004. 42: p. 125-130.

18. Sangshetti, J.N., et al., Quality by design approach: Regulatory need. Arabian Journal of Chemistry, 2017. 10: p. S3412-S3425.

19. Khurana, R.K., et al., Enhancing biopharmaceutical attributes of phospholipid complex-loaded nanostructured lipidic carriers of mangiferin: Systematic development, characterization and evaluation. International Journal of Pharmaceutics, 2017. 518(1-2): p. 289-306. 
20. Aslam, M., et al., Application of Box-Behnken design for preparation of glibenclamide loaded lipid based nanoparticles: Optimization, in vitro skin permeation, drug release and in vivo pharmacokinetic study. Journal of Molecular Liquids, 2016. 219: p. 897908.

21. Muppidi, K., et al., Development and stability studies of novel liposomal vancomycin formulations. ISRN Pharm, 2012. 2012: p. 636743.

22. Rizwanullah, M., J. Ahmad, and S. Amin, Nanostructured Lipid Carriers: A Novel Platform for Chemotherapeutics. Current Drug Delivery, 2016. 13(1): p. 4-26.

23. Thang, L.Q., N.D. Hanh, and D.Q. Duong, Study on cause-effect relations and optimization of exemestane-loaded nanostructured lipid carriers. International Journal of Pharmacy and Pharmaceutical Sciences, 2017. 9 (5), p. 68-74.

24. Ghassemi, S., et al., Labrasol-Enriched Nanoliposomal Formulation: Novel Approach to Improve Oral Absorption of Water-Insoluble Drug, Carvedilol. AAPS PharmSciTech, 2018. 19(7): p. 2961-2970.

25. Wang, F., et al., Optimization of methazolamide-loaded solid lipid nanoparticles for ophthalmic delivery using Box-Behnken design. Journal of Liposome Research, 2014. 24(3): p. 171-181.

26. Malzert-Fréon, A., et al., Influence of the introduction of a solubility enhancer on the formulation of lipidic nanoparticles with improved drug loading rates. European Journal of Pharmaceutics and Biopharmaceutics, 2010. 75(2): p. 117-127.

27. Myers, R.H., D.C. Montgomery, and C.M. Anderson-Cook, Response surface methodology: process and product optimization using designed experiments. John Wiley \& Sons, 2016.

28. Taneja, N. and K.K. Singh, Rational design of polysorbate 80 stabilized human serum albumin nanoparticles tailored for high drug loading and entrapment of irinotecan. International Journal of Pharmaceutics, 2018. 536(1): p. 82-94.

29. Velmurugan, R. and S. Selvamuthukumar, Development and optimization of ifosfamide nanostructured lipid carriers for oral delivery using response surface methodology. Applied Nanoscience, 2016. 6(2): p. 159-173.

30. Garg, N., et al., Quality by Design (QbD)-enabled development of aceclofenac loadednano structured lipid carriers (NLCS): An improved dermatokinetic profile for inflammatory disorder(s). International Journal of Phrmaceutics, 2016. 517. p. 413431.

31. Niculae, G., et al., Optimization of lipid nanoparticles composition for sunscreen encapsulation. U.P.B. UPB Scientific Bulletin, Series B: Chemistry and Materials Science 2013. 75(3): p. 80-92.

32. Jain, K., S. Sood, and K. Gowthamarajan, Optimization of artemether-loaded NLC for intranasal delivery using central composite design. Drug delivery, 2015. 22(7): p. 940954.

33. Das, S., W.K. Ng, and R.B.H. Tan, Are nanostructured lipid carriers (NLCs) better than solid lipid nanoparticles (SLNS): development, characterizations and comparative evaluations of clotrimazole-loaded SLNs and NLCs? 2012. European Journal of Pharmceutical Sciences, 2012. 47(1): p. 139-151.

34. Pimentel-Moral, S., et al., Polyphenols-enriched Hibiscus sabdariffa extract-loaded nanostructured lipid carriers (NLC): Optimization by multi-response surface methodology. Journal of Drug Delivery Science and Technology, 2019. 49: p. 660-667.

35. Savić, V., et al., Tacrolimus-loaded lecithin-based nanostructured lipid carrier and nanoemulsion with propylene glycol monocaprylate as a liquid lipid: Formulation characterization and assessment of dermal delivery compared to referent ointment. International Journal of Pharmaceutics, 2019. 569: p. 118624. 
36. Agrawal, Y., K.C. Petkar, and K.K. Sawant, Development, evaluation and clinical studies of Acitretin loaded nanostructured lipid carriers for topical treatment of psoriasis. International Journal of Pharmaceutics, 2010. 401(1-2): p. 93-102.

37. Witayaudom, P. and U. Klinkesorn, Effect of surfactant concentration and solidification temperature on the characteristics and stability of nanostructured lipid carrier (NLC) prepared from rambutan (Nephelium lappaceum L.) kernel fat. Journal of Colloid and Interface Science, 2017. 505: p. 1082-1092.

38. Badie, H. and H. Abbas, Novel small self-assembled resveratrol-bearing cubosomes and hexosomes: Preparation, charachterization, and ex vivo permeation. Drug development and Industrial Pharmacy, 2018. 44(12): p. 2013-2025.

39. Pandita, D., et al., Solid lipid nanoparticles enhance oral bioavailability of resveratrol, a natural polyphenol. Food Research International, 2014. 62: p. 1165-1174.

40. Ibrahim, N., I. Raman, and M.R. Yusop, Effects of functional group of non-ionic surfactants on the stability of emulsion. Malaysian Journal of Analytical Sciences, 2015. 19(1): p. 261-267.

41. Sanad, R.A., et al., Formulation of a Novel Oxybenzone-Loaded Nanostructured Lipid Carriers (NLCs). AAPS PharmaSciTech, 2010, 11: p.1684-94.

42. Agarwal.A., Kharb.V., and Anand. S.V., Process optimisation, characterisation and evaluation of resveratrol-phospholipid complexes using Box-Behnken statistical design. International Current Pharmaceutical Journal 2014. 3(7): p. 301-308.

43. Neves, A.R., et al., Novel resveratrol nanodelivery systems based on lipid nanoparticles to enhance its oral bioavailability. Int J Nanomedicine, 2013. 8: p. 17787.

44. Emami, J., et al., Formulation of LDL Targeted Nanostructured Lipid Carriers Loaded with Paclitaxel: A Detailed Study of Preparation, Freeze Drying Condition, and In Vitro Cytotoxicity. Journal of Nanomaterials, 2012. 2012: p. 10.

45. Patel, M. and K. Sawant, A Quality by Design Concept on Lipid Based Nanoformulation Containing Antipsychotic Drug: Screening Design and Optimization using Response Surface Methodology. Jornal of Nanomedicine and Nanotechnology, 2017. 8(3): p. 442.

46. de Carvalho, S.M., et al., Optimization of $\alpha$-tocopherol loaded solid lipid nanoparticles by central composite design. Industrial crops and products. Industrial crops and Products 2013. 49: p. 278-285.

47. Pople, P.V. and K.K. Singh, Development and evaluation of colloidal modified nanolipid carrier: Application to topical delivery of tacrolimus. European Journal of Pharmaceutics and Biopharmaceutics, 2011. 79(1): p. 82-94.

48. Severino, P., et al., Crystallinity of Dynasan ${ }^{\circledR} 114$ and Dynasan ${ }^{\circledR} 118$ matrices for the production of stable Miglyol $\mathbb{R}-l o a d e d$ nanoparticles. Journal of thermal analysis and calorimetry, 2011. 108(1): p. 101-108.

49. Iqbal, B., J. Ali, and S. Baboota, Silymarin loaded nanostructured lipid carrier: from design and dermatokinetic study to mechanistic analysis of epidermal drug deposition enhancement. Journal of Molecular Liquids, 2018. 255: p. 513-529.

50. Kobierski, S., et al., Resveratrol nanosuspensions for dermal application-production, characterization, and physical stability. Die Pharmazie-An International Journal of Pharmaceutical Sciences, 2009. 64(11): p. 741-747.

51. Hao, J., et al., Development and optimization of baicalin-loaded solid lipid nanoparticles prepared by coacervation method using central composite design. European Journal of Pharmaceutical Sciences, 2012. 47(2): p. 497-505.

52. Gokce, E.H., et al., Resveratrol-loaded solid lipid nanoparticles versus nanostructured lipid carriers: evaluation of antioxidant potential for dermal applications. International Journal of Nanomedicine, 2012. 7: p. 1841. 
53. Pople, P.V. and K.K. Singh, Targeting tacrolimus to deeper layers of skin with improved safety for treatment of atopic dermatitis. International Journal of Pharmaceutics, 2010. 398(1-2): p. 165-178.

54. Soleimanian, Y., et al., Propolis wax nanostructured lipid carrier for delivery of $\beta$ sitosterol: effect of formulation variables on physicochemical properties. Food chemistry, 2018. 260: p. 97-105.

55. Garti, N., J. Schlichter, and S. Sarig, DSC studies concerning polymorphism of saturated monoacid triglycerides in the presence of food emulsifiers. Lipid/Fett, 1988. 90(8): p. 295-299.

56. Ramasamy, T., et al., Layer-by-layer coated lipid-polymer hybrid nanoparticles designed for use in anticancer drug delivery. Carbohydrate Polymers, 2014. 102: p. 653-661.

57. Azhar Shekoufeh Bahari, L. and H. Hamishehkar, The Impact of Variables on Particle Size of Solid Lipid Nanoparticles and Nanostructured Lipid Carriers; A Comparative Literature Review. Advanced Pharmaceutical Bulletin, 2016. 6(2): p. 143-151.

58. Elnaggar, Y.S.R., M.A. El-Massik, and O.Y. Abdallah, Fabrication, appraisal, and transdermal permeation of sildenafil citrate-loaded nanostructured lipid carriers versus solid lipid nanoparticles. International Journal of Nanomedicine, 2011. 6: p. 3195-3205.

59. Panda, R. and K. Kuotsu, Fabrication, characterization, and in vitro evaluation of pegylated glyceride labrasol® nanostructured lipid carrier composites of methotrexate: the pathway to effective cancer therapy. Asian Journal of Pharmceutical and Clinical Research, 2019. 12(6): p. 229-237.

60. Driscoll, D.F., et al., The influence of medium-chain triglycerides on the stability of allin-one formulations. International Journal of Pharmaceutics, 2002. 240(1-2): p. 1-10.

61. Wu, L., J. Zhang, and W. Watanabe, Physical and chemical stability of drug nanoparticles. Advanced Drug Delivery Reviews, 2011. 63(6): p. 456-469.

62. Zhang, Z. and D.J. McClements, Overview of Nanoemulsion Properties: Stability, Rheology, and Appearance, in Nanoemulsions. 2018, Elsevier. p. 21-49.

63. Yuan, H., et al., Preparation and characteristics of nanostructured lipid carriers for control-releasing progesterone by melt-emulsification. Colloids and Surfaces B: Biointerfaces, 2007. 60(2): p. 174-179.

64. Araújo, J., et al., Nanostructured lipid carriers for triamcinolone acetonide delivery to the posterior segment of the eye. Colloids and Surfaces B: Biointerfaces, 2011. 88(1): p. 150-157.

65. Zupančič, Š., Z. Lavrič, and J. Kristl, Stability and solubility of trans-resveratrol are strongly influenced by $\mathrm{pH}$ and temperature. European Journal of Pharmaceutics and Biopharmaceutics, 2015. 93: p. 196-204. 


\section{FIGURES}

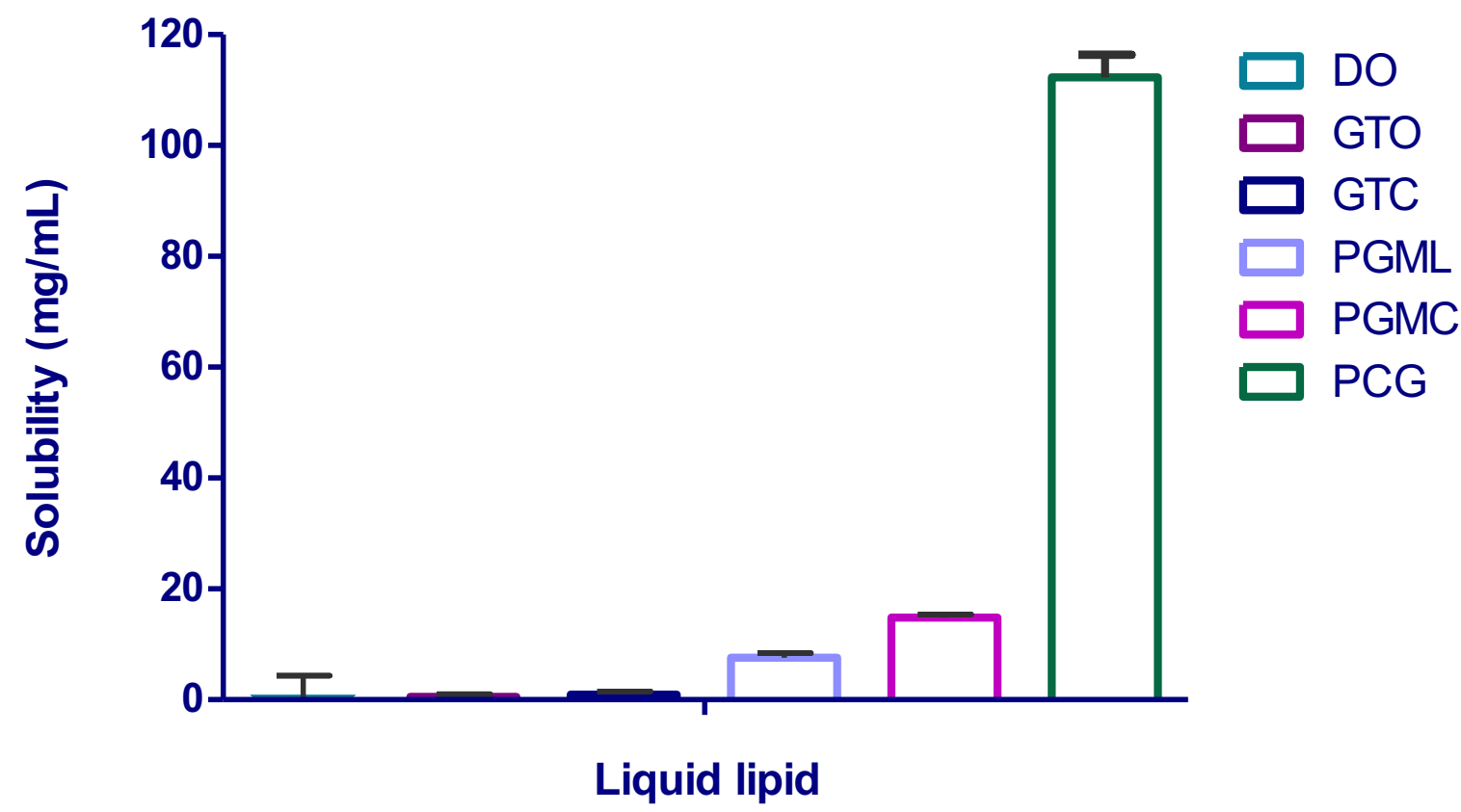

Figure 1. Solubility studies of RES in various lipid lipids $(n=3)$, error bars represent the standard error of the mean 
a

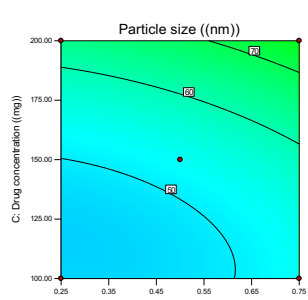

A. Liquid lipid oncentration (1\%\%)
Particle size ((nm))

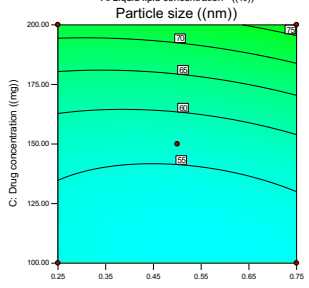

A. Liquid lipid concentration (1\%)
Particle size ((n)

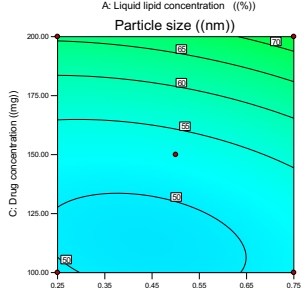

A. Liquid lipid concentration ((\%))

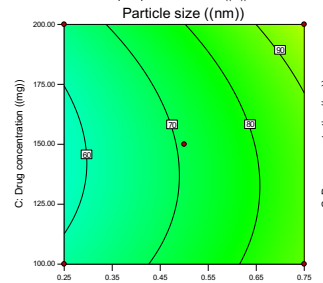

Liquid lipid concentration (1\%)
Particle size ((nm))

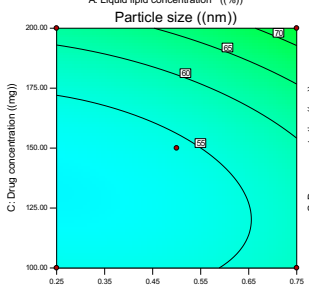

A. Liquidi lipid concentration (\%(\%)
Particle size (nm))

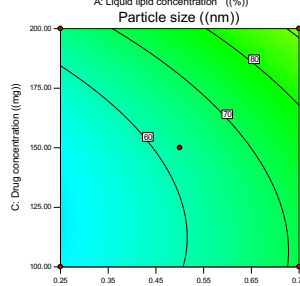

$\prod_{24.98}^{131.4}$ b
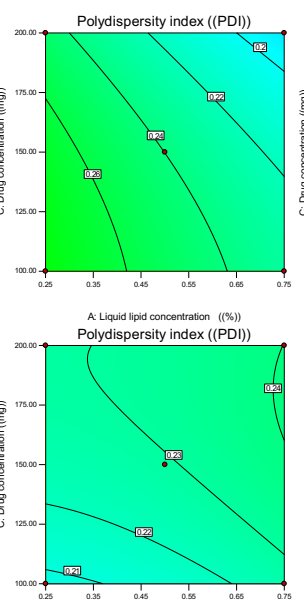

A. Liquid lipid concentration (1\%)

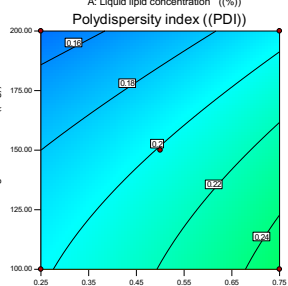

A. Liquid lipid concentrataion ((\%))
Polydispersity index ((PDI)

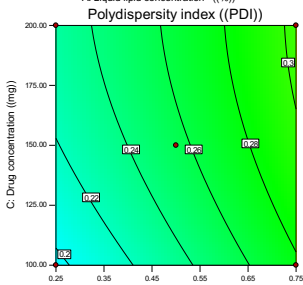

A. Liquid lipid concentration (\%\%))
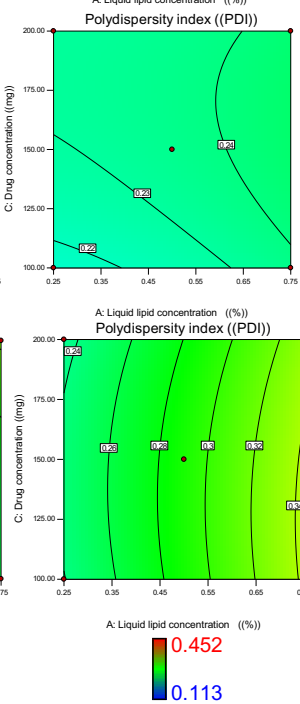

c

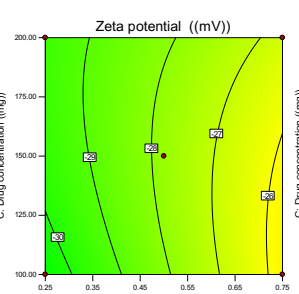

Liquid lipid concentration (1\%)

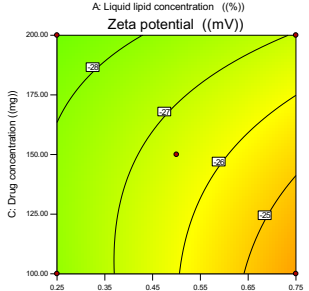

A. Liquid lipid concentration ((\%)

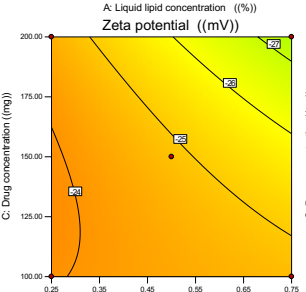

A. Liquid lipid concentration (1\%))
Zeta potential ((mV))
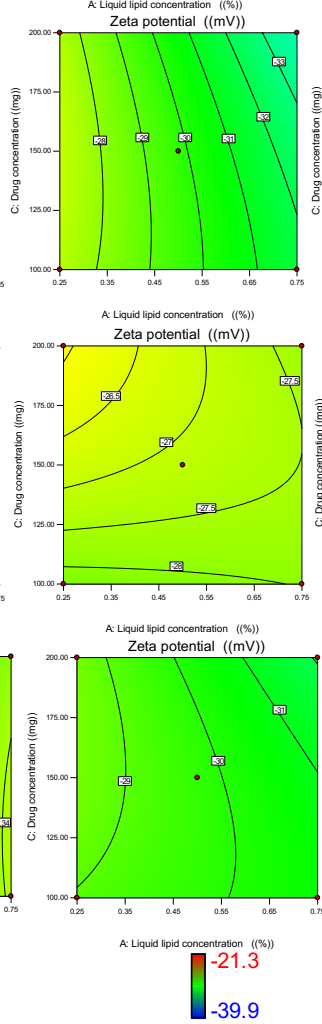

d

e

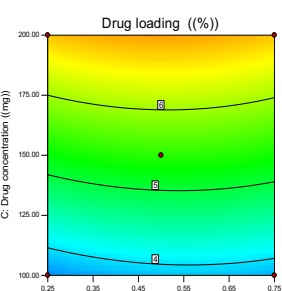

Liquid lipid concentration (18))
Entrapment efficiency

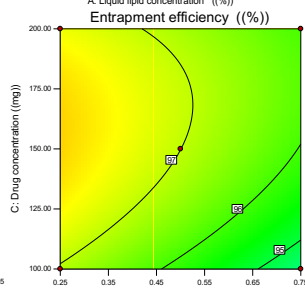

A. Liquid lipid concentration $(\%(\%))$
Entrapment efficiency $((\%))$

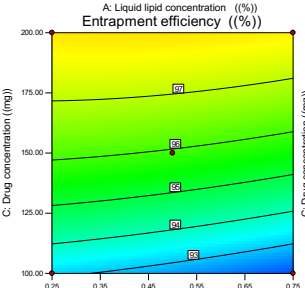

A. Liquid lipid concentration (\%\%))
Entrapment efficiency ((\%)

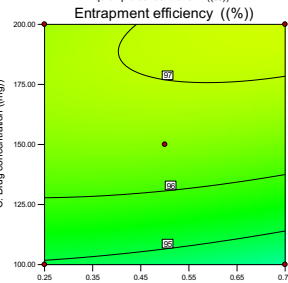

A. Liquid lipid concentration (\%(\%))
Entrapment efficiency $((\%))$
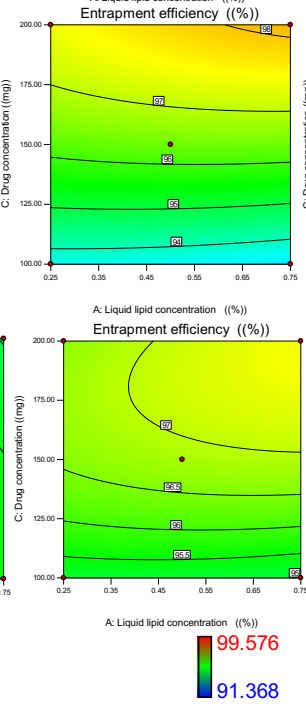

GTC

DO

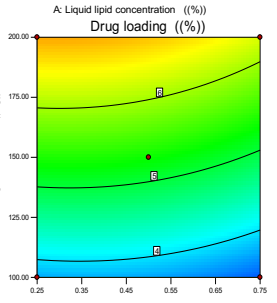

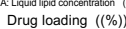

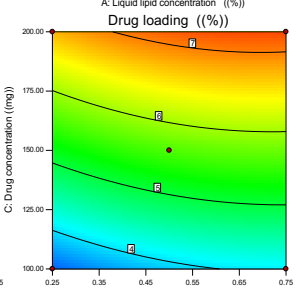

Liquid lipid concentration ((\%))
Drug loading $((\%))$

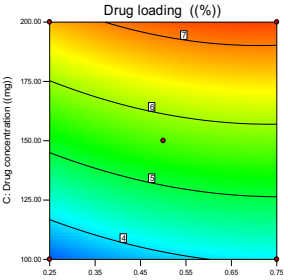

A: Liquid lipid concentration ((\%))
Drug loading ((\%))

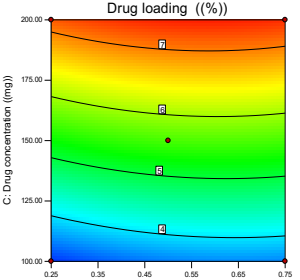

Liquid lipid concentration (1(\%))
Drug loading ((\%))

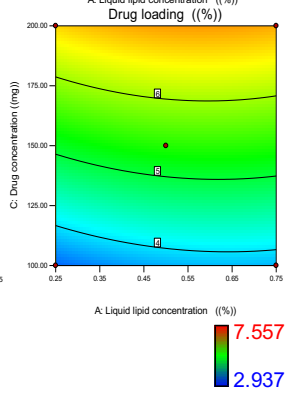

PGMC

GTO

PGML

Figure 2. A) 2-D contour plots showing the effect of liquid lipid concentration and amount of drug at medium level of Tween 80 concentration on various response variables of NLCs: (a)PS, (b) PDI, (c) ZP, (d) EE, (e) DL 
a

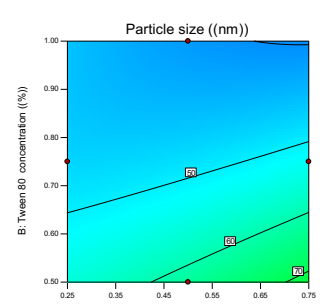

A L Liquid lipid concentration (t)

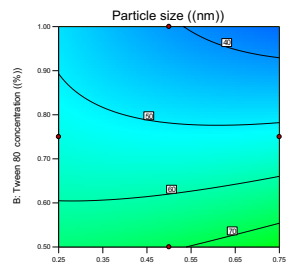

A : Liquid lipid concentration (1\%)
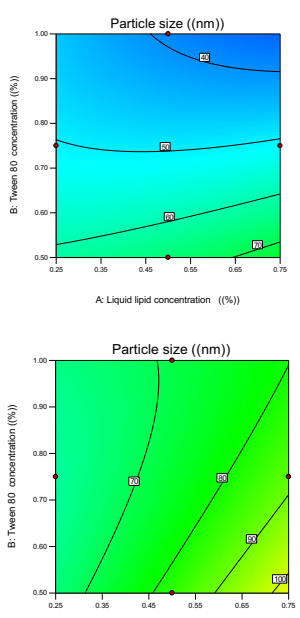

A. Liquid lipid concentration "(it)

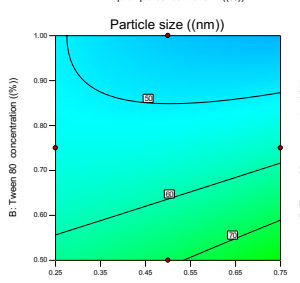

A. Liquid lipid concentration (\%\%)

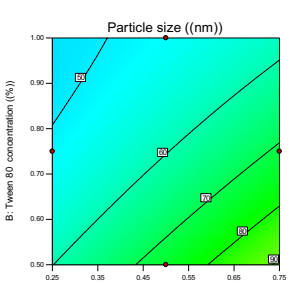

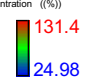

b
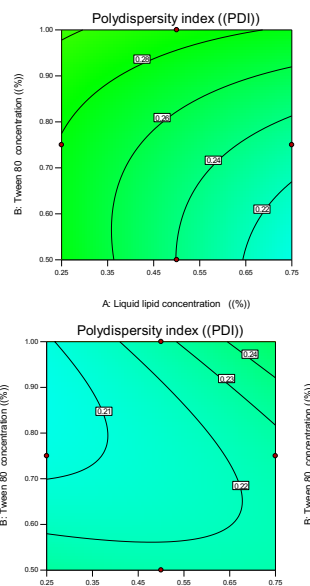

A: Liquid lipid concentration (1\%)
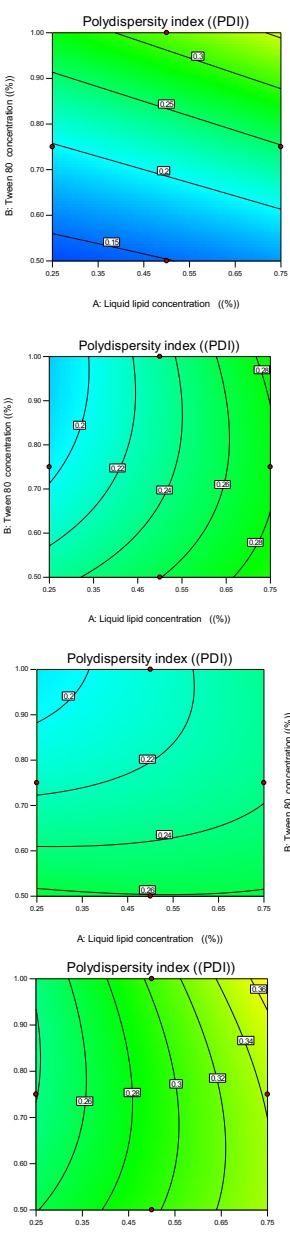

$\prod_{0.113}^{0.452}$ c

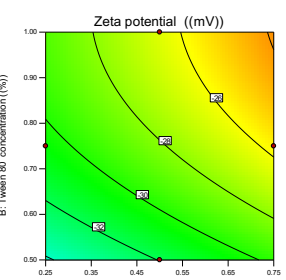

A. Liquid lipid concentration (1(\%))
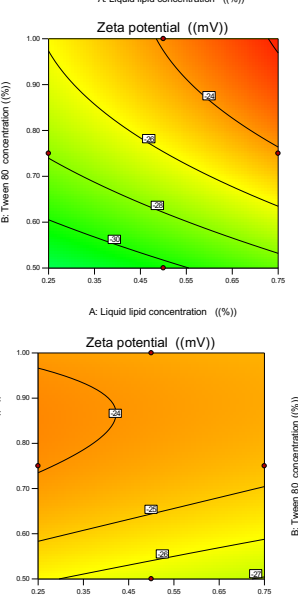

A. Liquid lipid concentration (1\%)

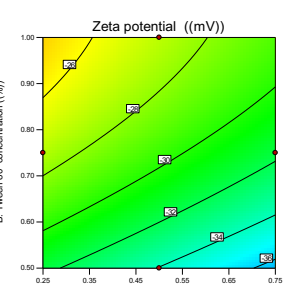

A. Liquid lipid conenntration (1\%)

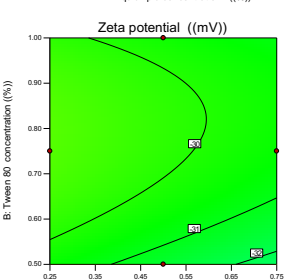

A : Liquid lipid concentration (1\%6)
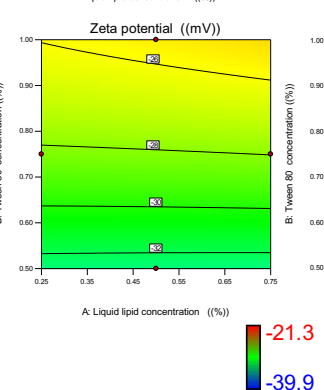

d
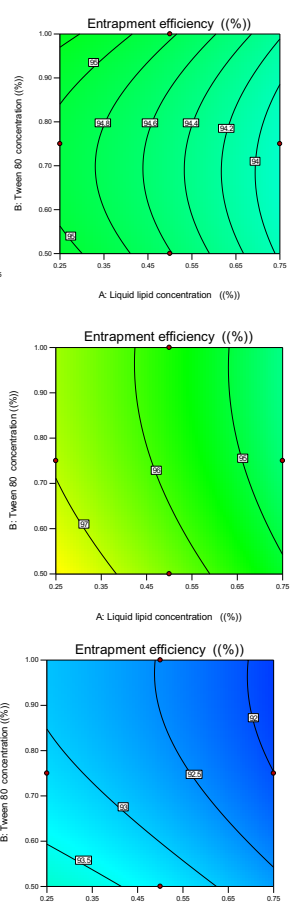

A. Liquid lipid concentration (1\%)
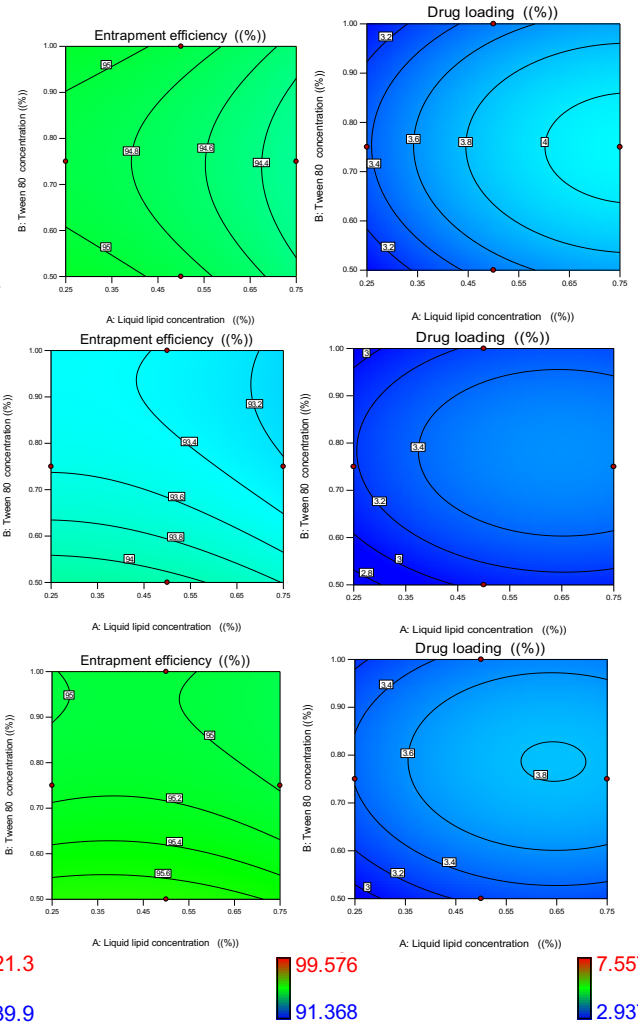

PCG

PGMC

GTO

PGML

Figure 2. B) 2-D contour plots showing the effect of liquid lipid and Tween 80 concentration at low level of drug on various response variables of NLCs: (a) PS, (b) PDI, (c) ZP, (d) EE, (e) DL 

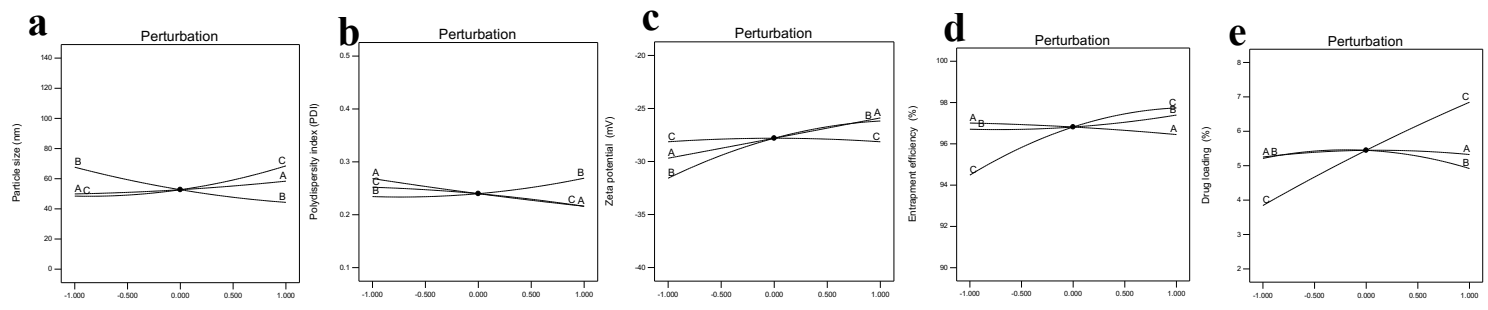

GTC
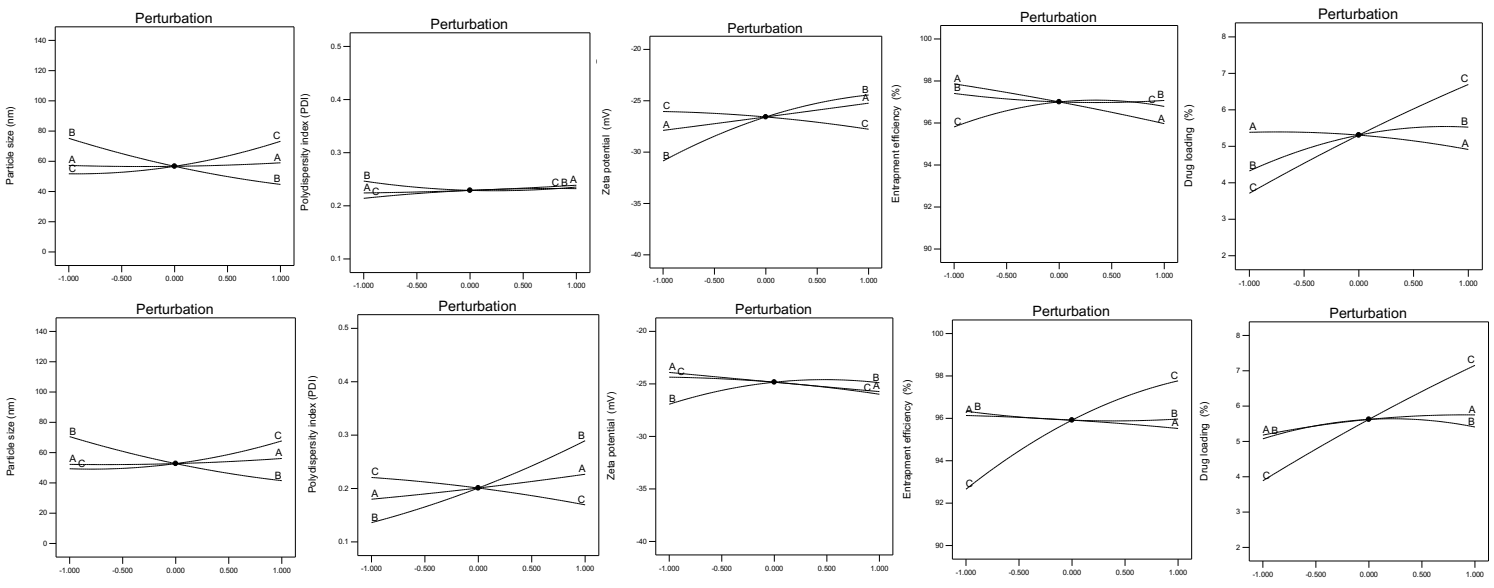

DO
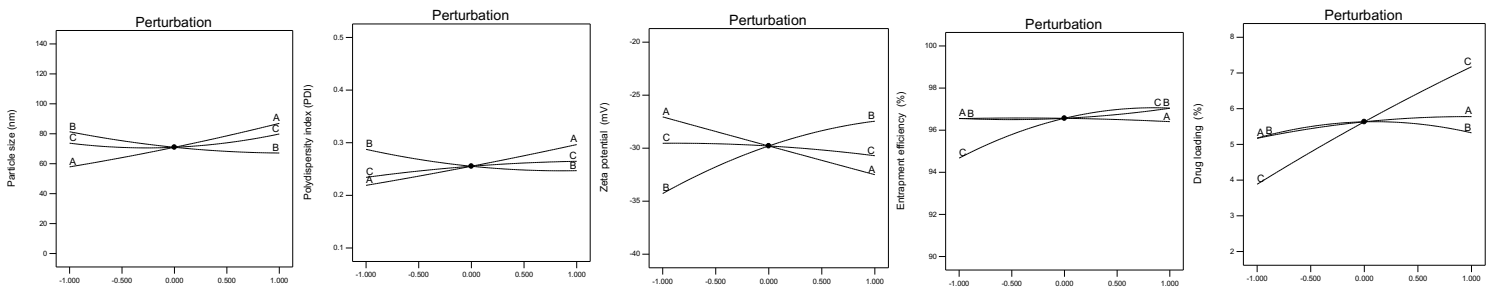

PCG
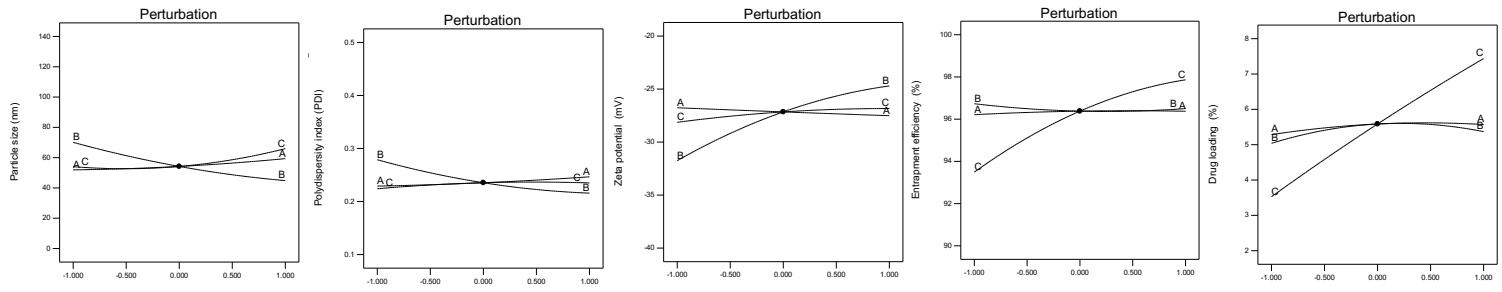

PGMC

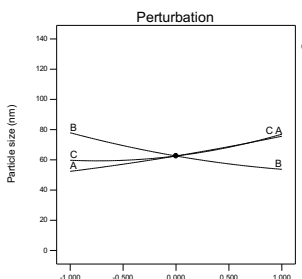

Particle size

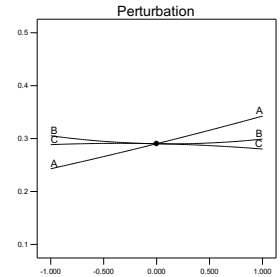

Polydispersity index

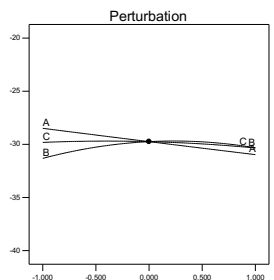

Zeta potential (mV)

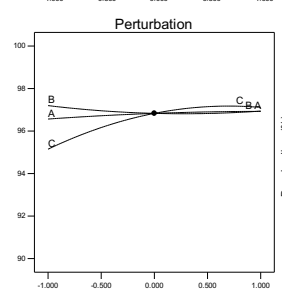

Entrapment efficiency (\%)

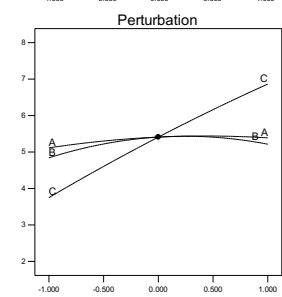

Drug loading (\%)

Figure 3) Perturbation plots showing the interactions between the liquid lipid concentration Tween 80 concentration and drug amount, all at medium level on variouse response variables of NLCs with different type of liquid lipids (a) PS, (b) PDI, (c) (ZP), (d) EE and (e) DL 

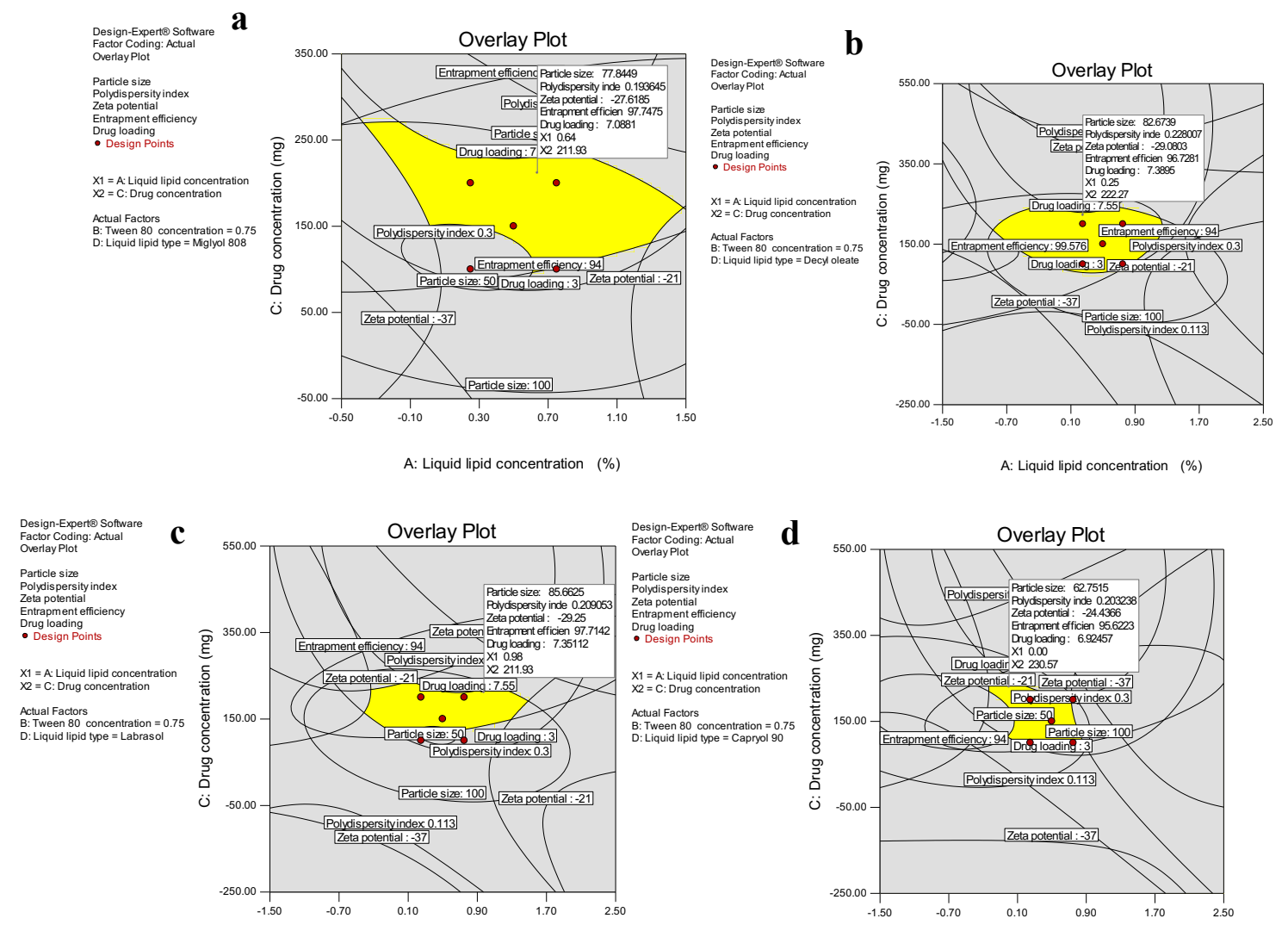

A: Liquid lipid concentration (\%)

A: Liquid lipid concentration (\%)
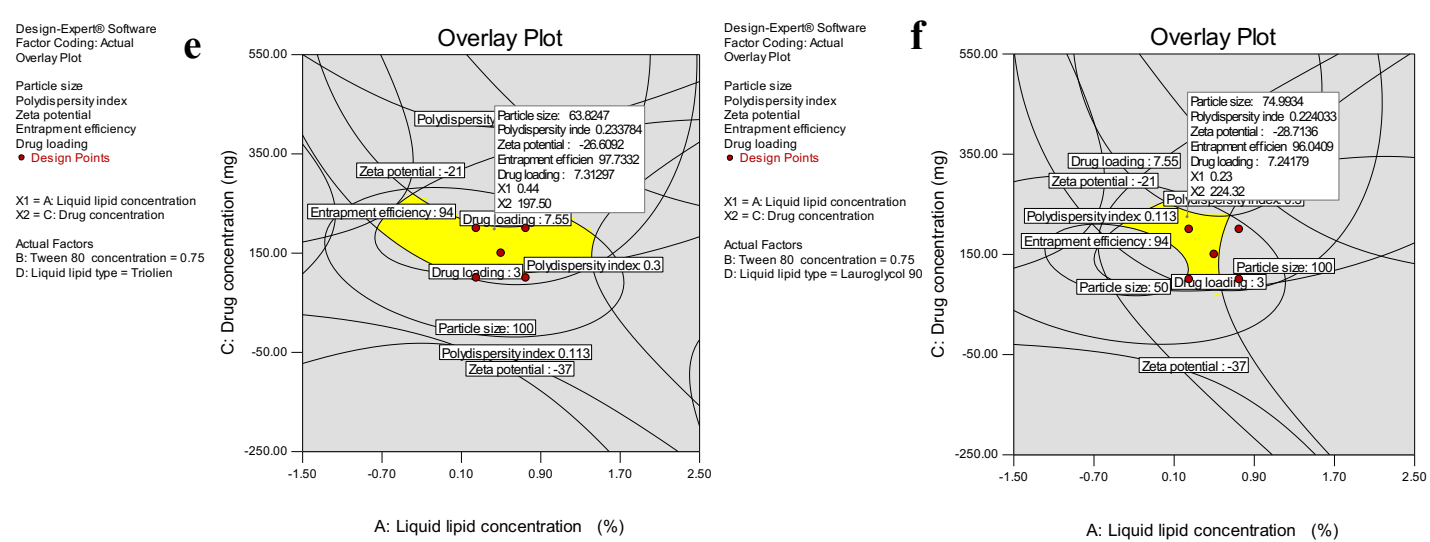

Figure 4) Overlay plots showing the location of optimized formulation with respect to the relationship between different responses to liquid lipid concentration and the drug amount for six different liquid lipid based NLCs (a) NLC with GTC, (b) NLC with DO, (c) NLC with PCG, (d) NLC with PGMC, (e) NLC with GTO (f) NLC with PGML 
a

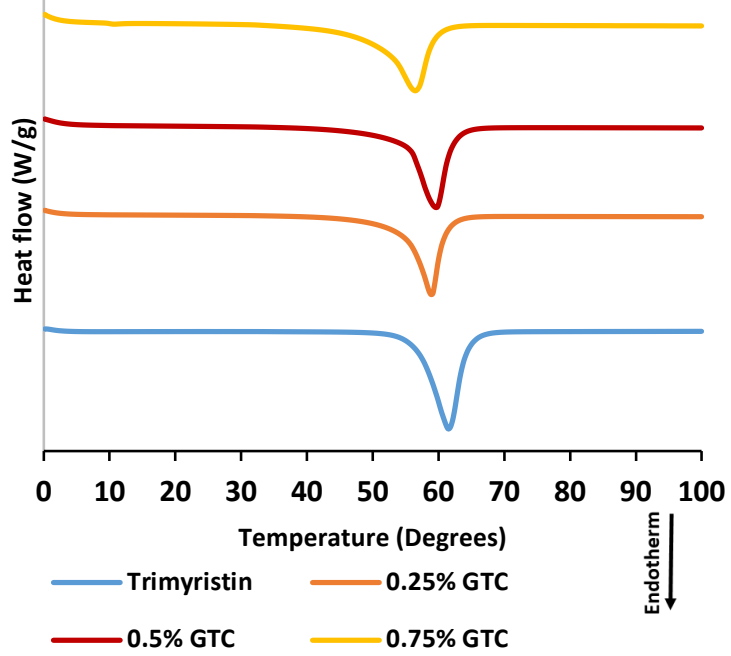

d

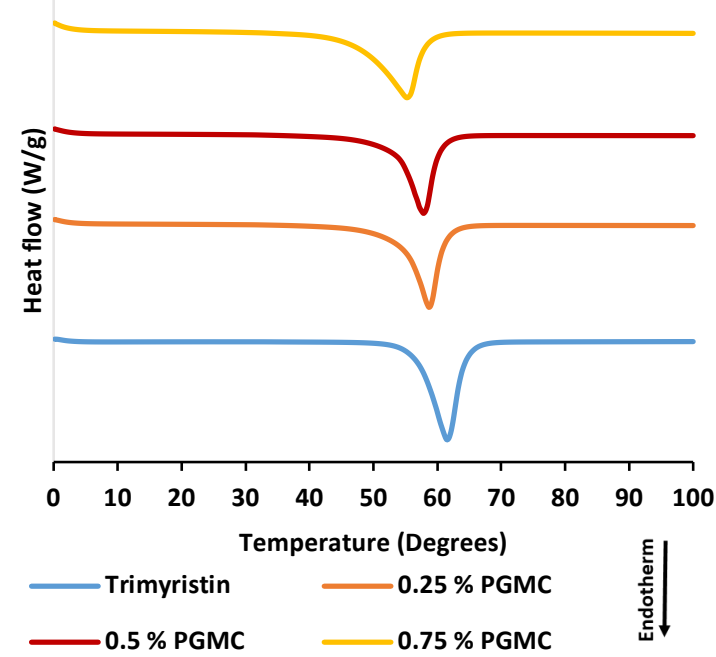

b

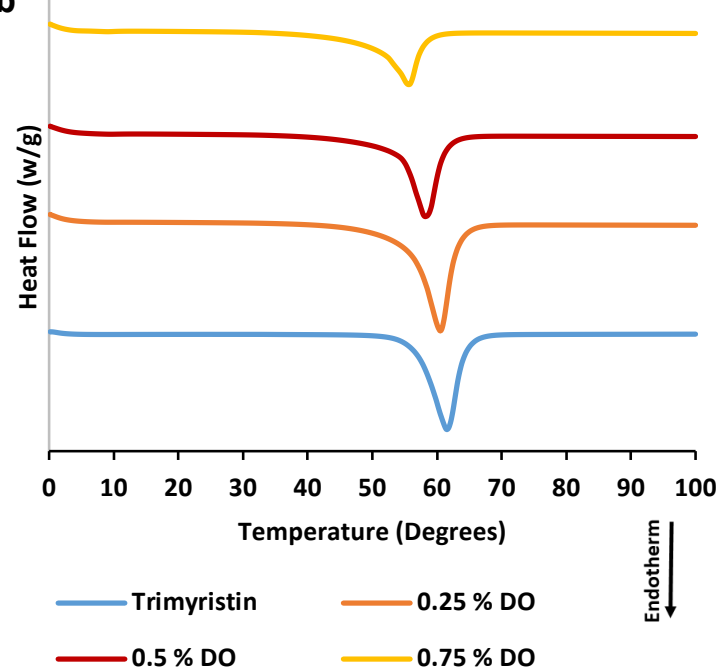

e

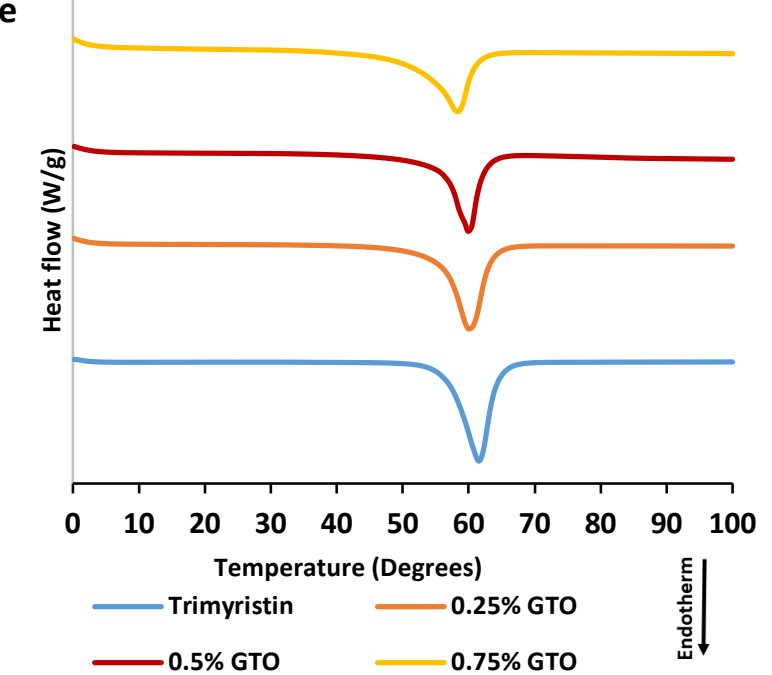

C

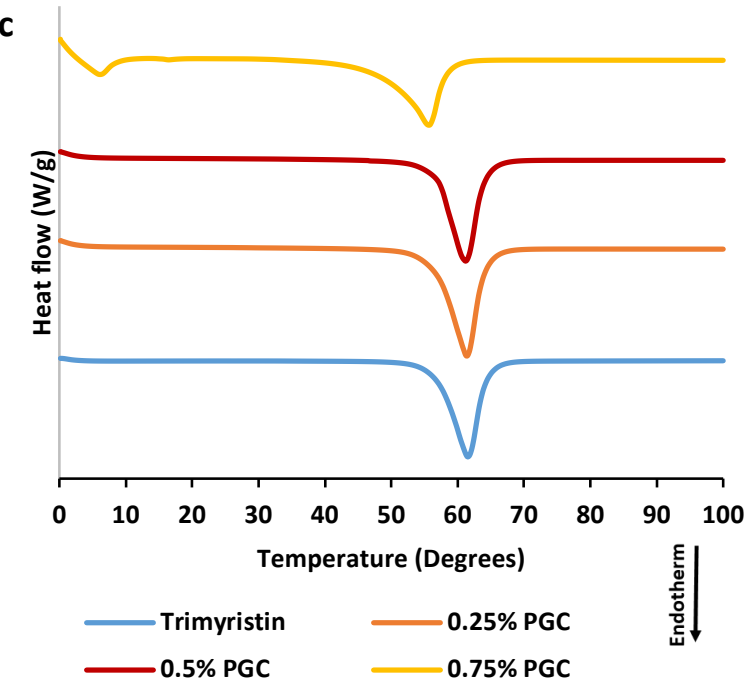

f

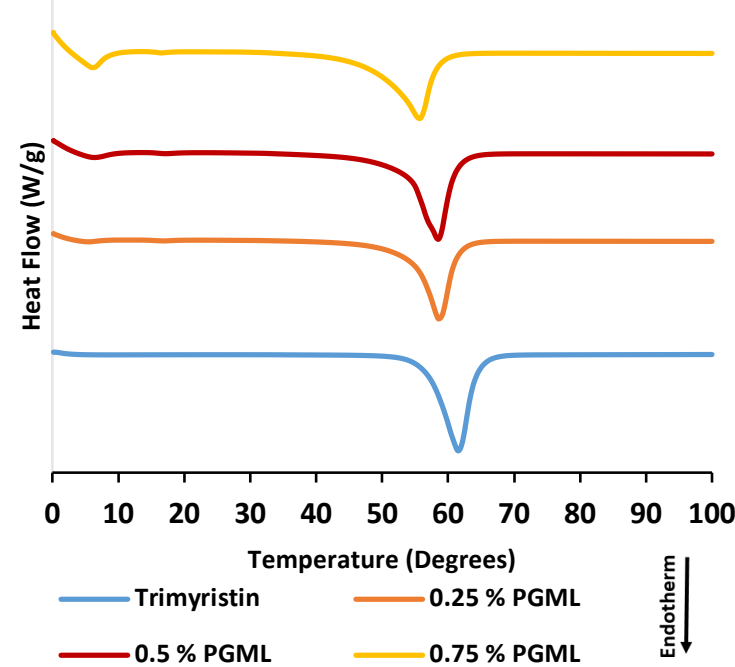

Figure 5A) DSC Thermograms showing the effect of oil concentration on the solid lipid a. GTC b. DO c. PCG d. PGMC e. GTO f. PGML 

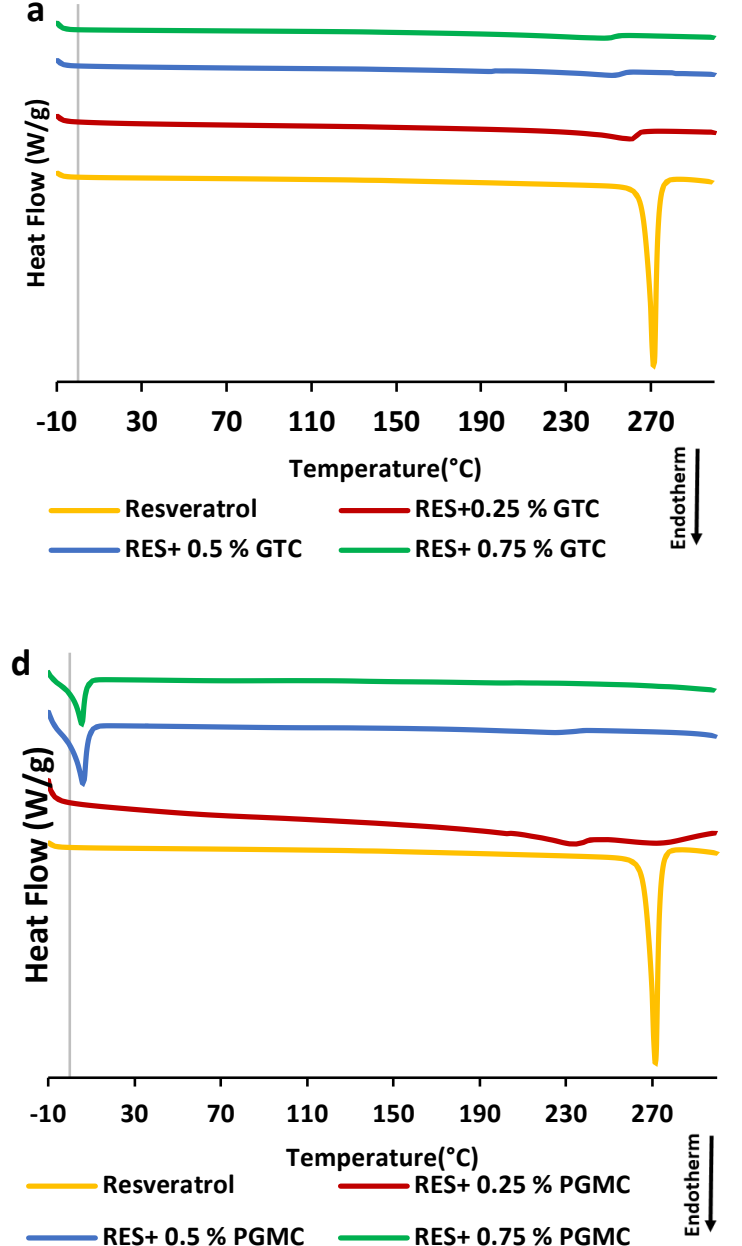

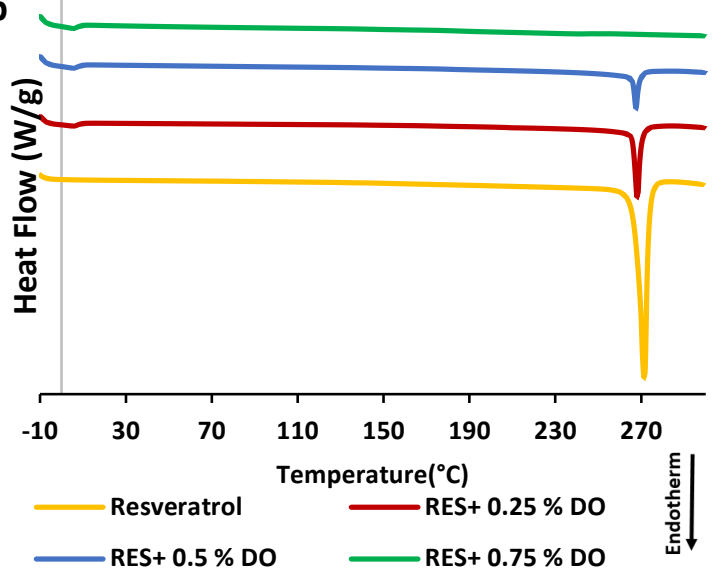

e

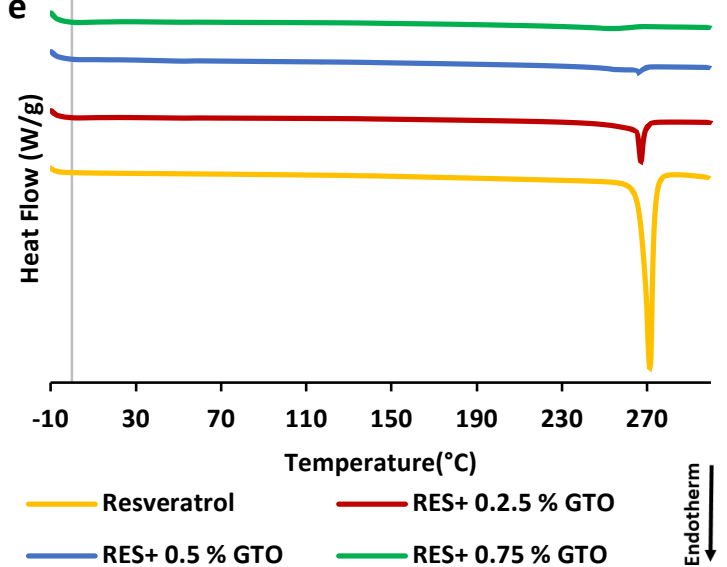

C
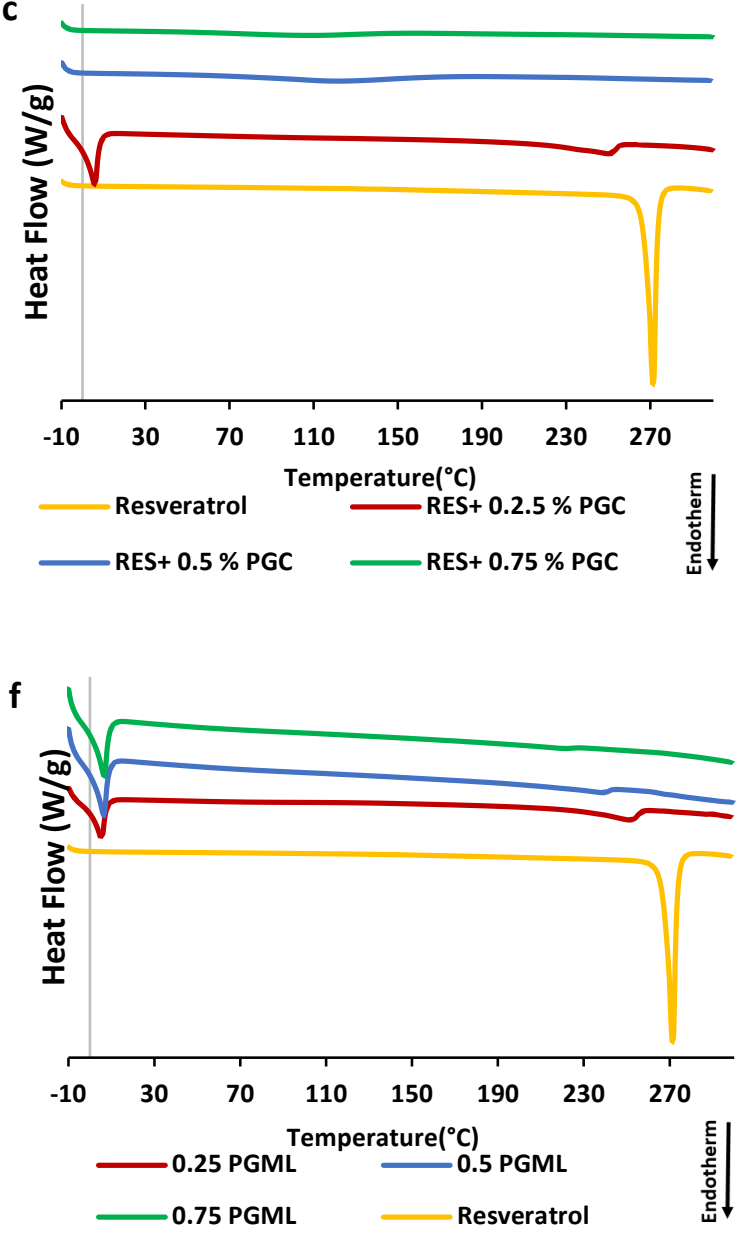

Figure 5.B) DSC Thermograms showing the effect of oil concentration on resveratrol solubility a. GTC, b. DO, c. PCG, d. PGMC, e. GTO, f. PGML 
a

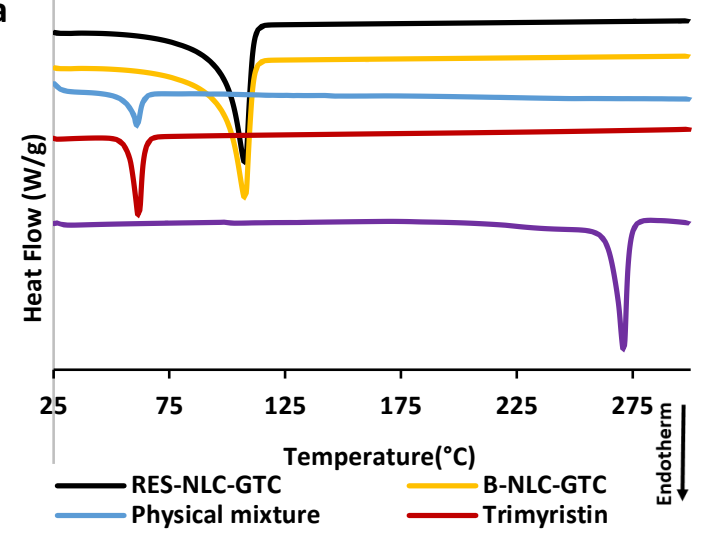

Physical mixture

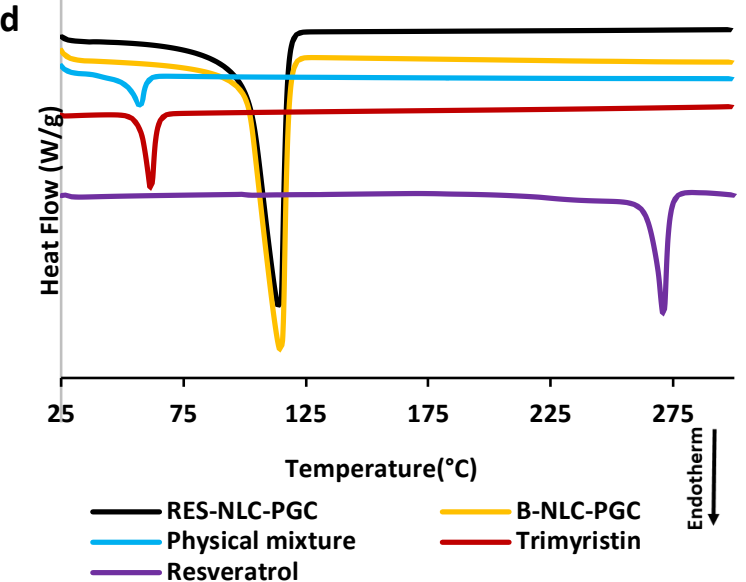

b

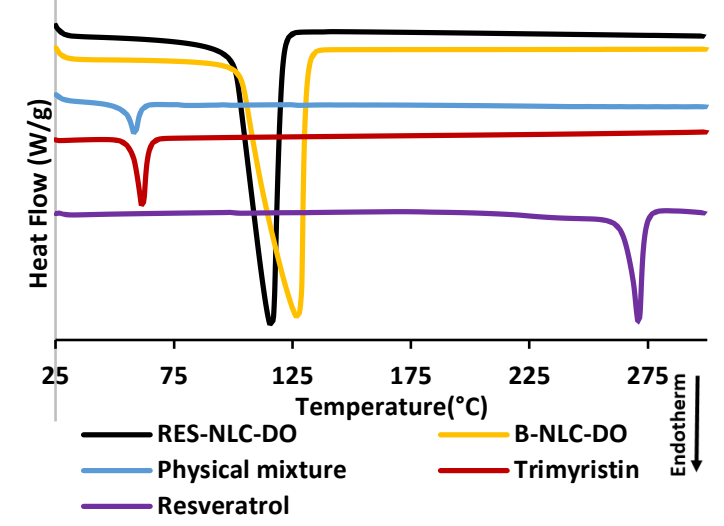

e

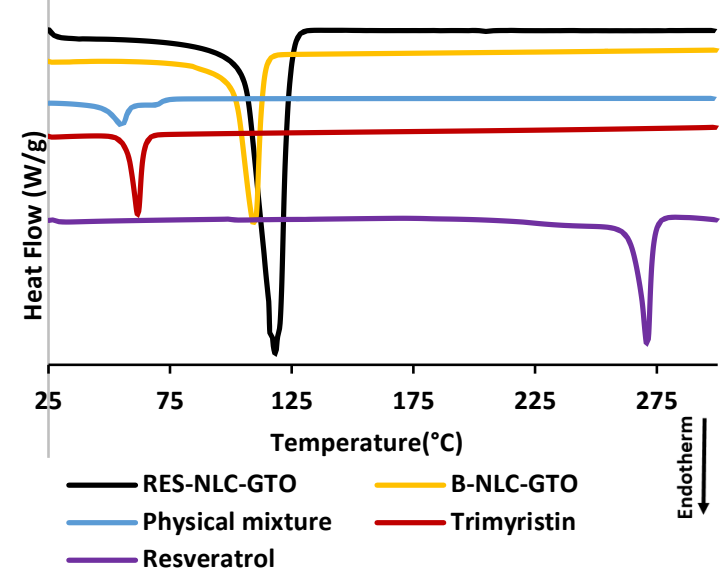

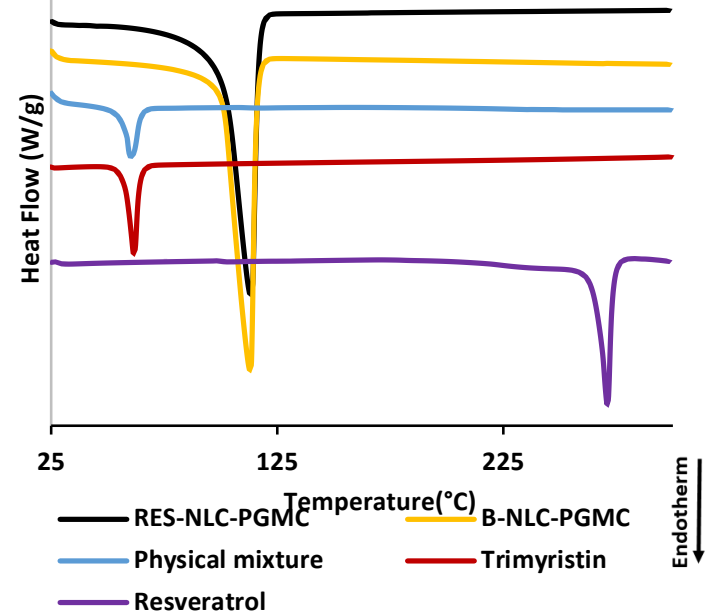

f

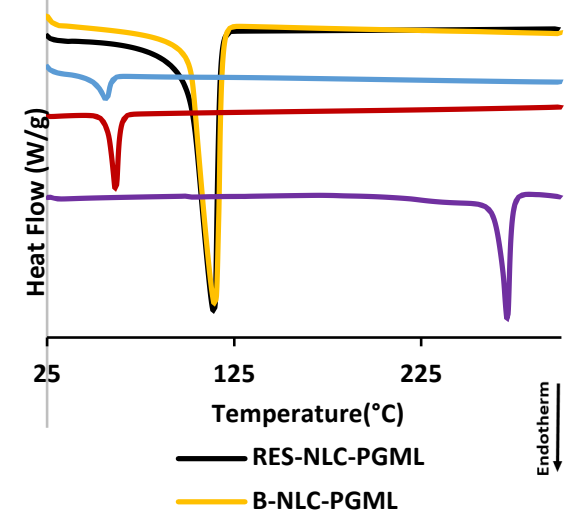

Figure 5.C) DSC heating thermogram of different RES-NLCs, B-NLCs, solid lipid, resveratrol and physical mixtures of a. GTC b. DO c. PCG d. PGMC e. GTO f. PGML 


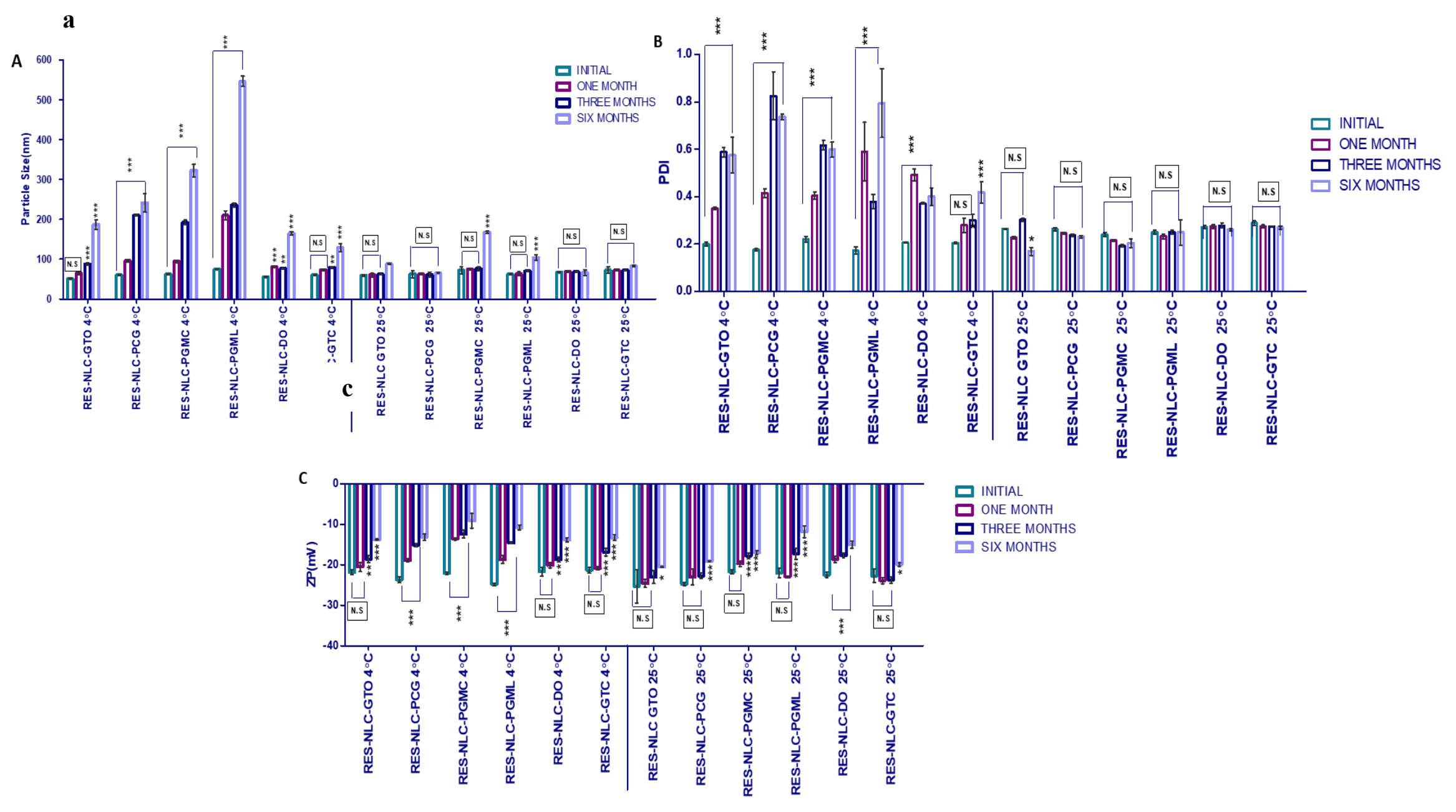




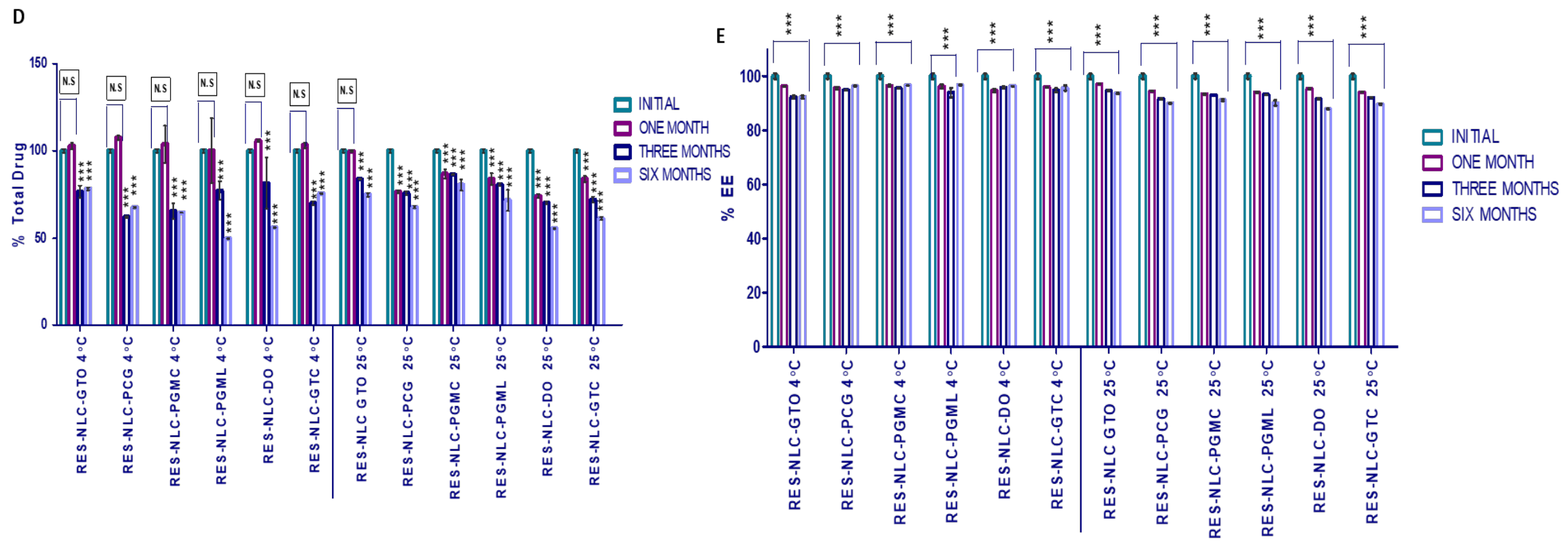

Figure 6. Storage stability of $\mathrm{NLC}$ at $4^{\circ} \mathrm{C}$ and $20^{\circ} \mathrm{C}$ at Initial (day zero), three months and six months and effect on (a) Particle size, (b) Polydispersity index, ( (c) Zeta potential, (d) Total drug and (e) Entrapment efficiency, error bars are standard error of the mean, Difference at $\mathbf{p}<0.05$ was considered statistically significant, $* *$ indicates $\mathbf{P}<0.001, * *, P<0.01$ and $* P<0.05$ 

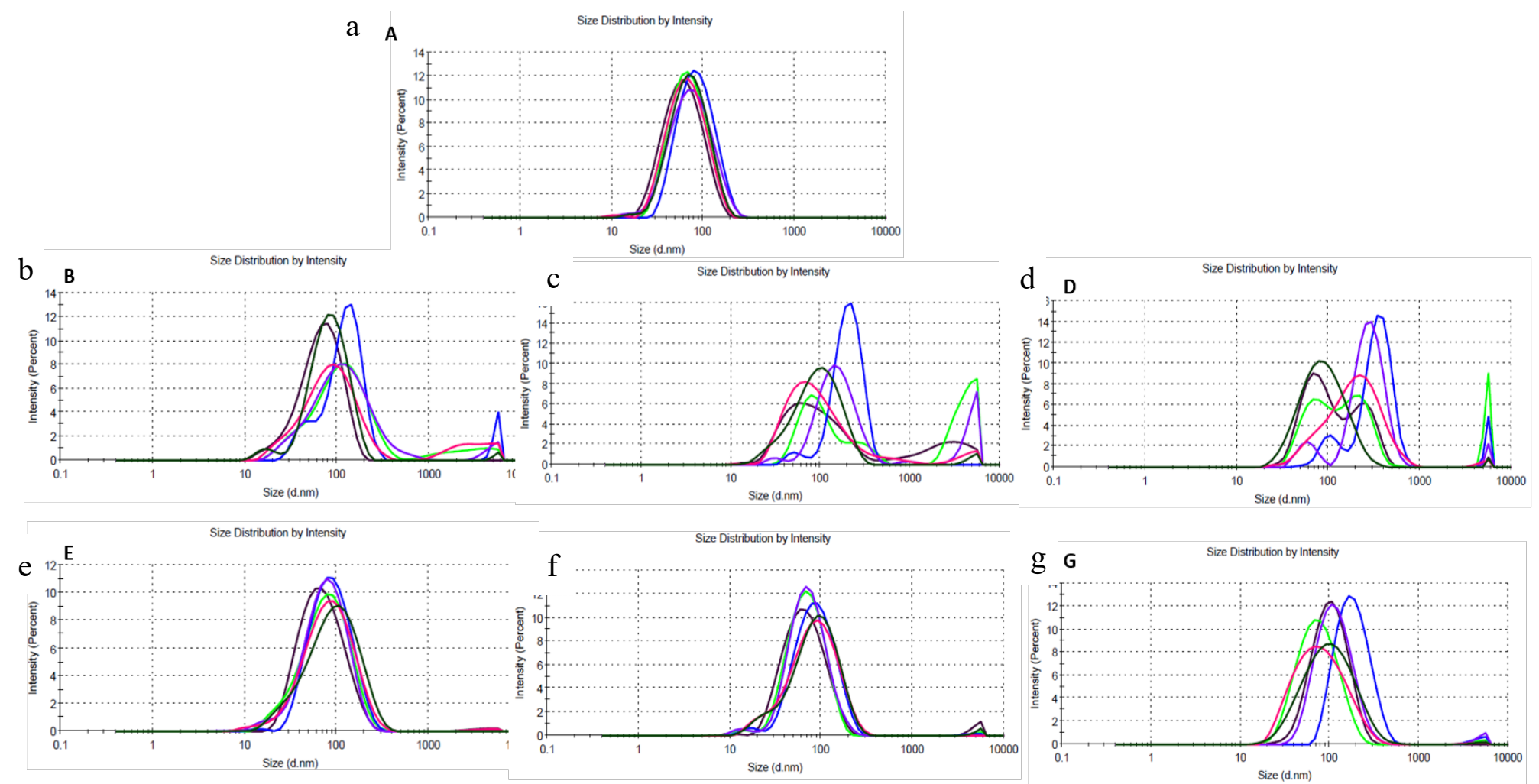

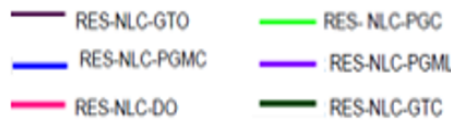


Figure 7 A) Overlay size distribution measured by zeta-sizer for stability of RES-NLCs after a. Initial (day zero) b. One, c. Three and d. Six months for each liquid lipid type stored at $4^{\circ} \mathrm{C}$ and e. One, f. Three and g. Six months stored at $20^{\circ} \mathrm{C}, \mathrm{n}=3$
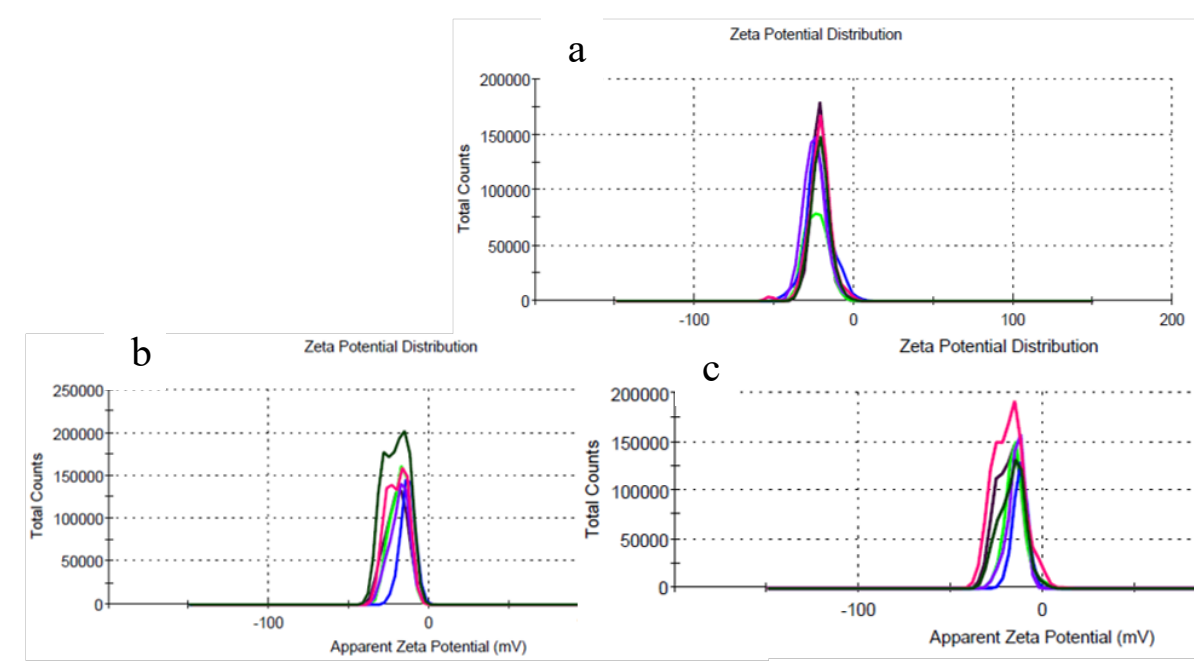

$-100$

Zeta Poten

C
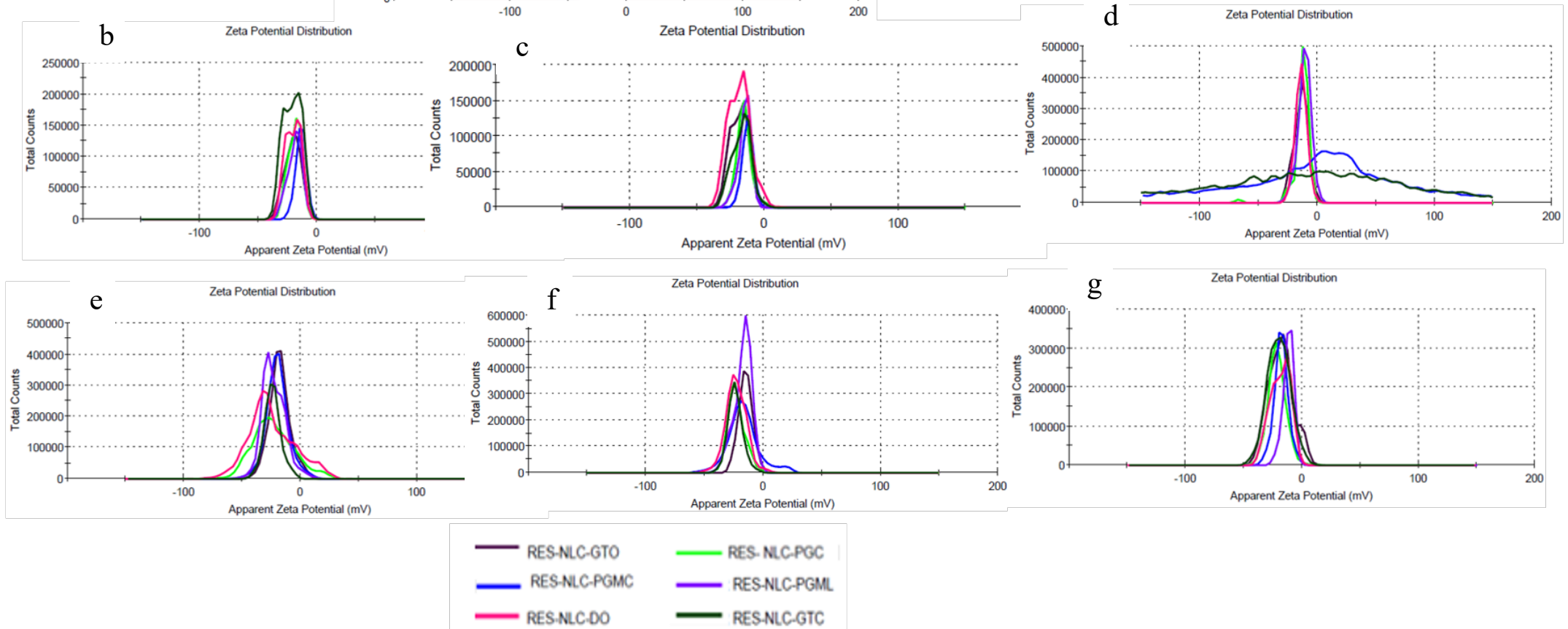

Figure 7 B) Overlay zeta potential measured by zeta-sizer for stability after (a) Initial (day zero) (b). One, (c). Three and D. Six months for each liquid lipid type stored at $4^{\circ} \mathrm{C}$ and (e). One, (f). Three and (g). Six months stored at $20^{\circ} \mathrm{C}, \mathrm{n}=3$ 


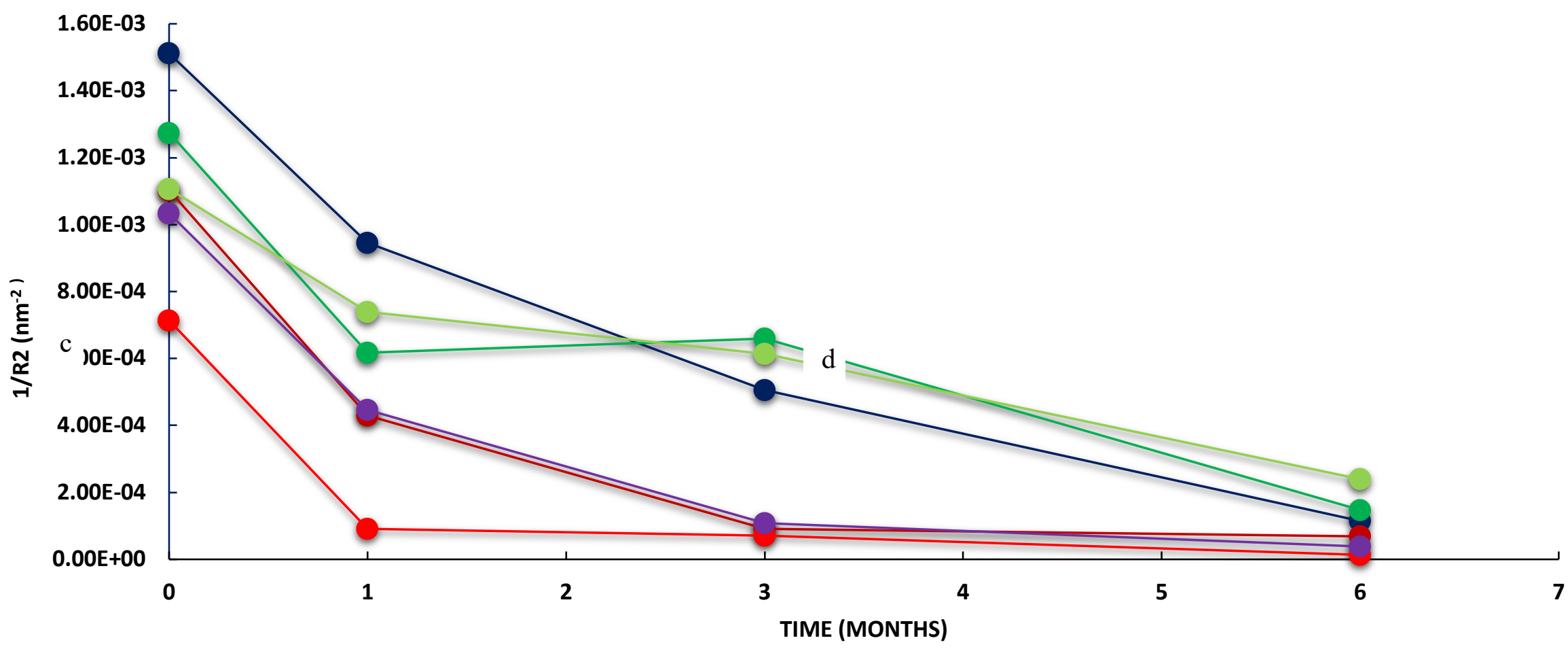

- RES-NLC-GTO 
b

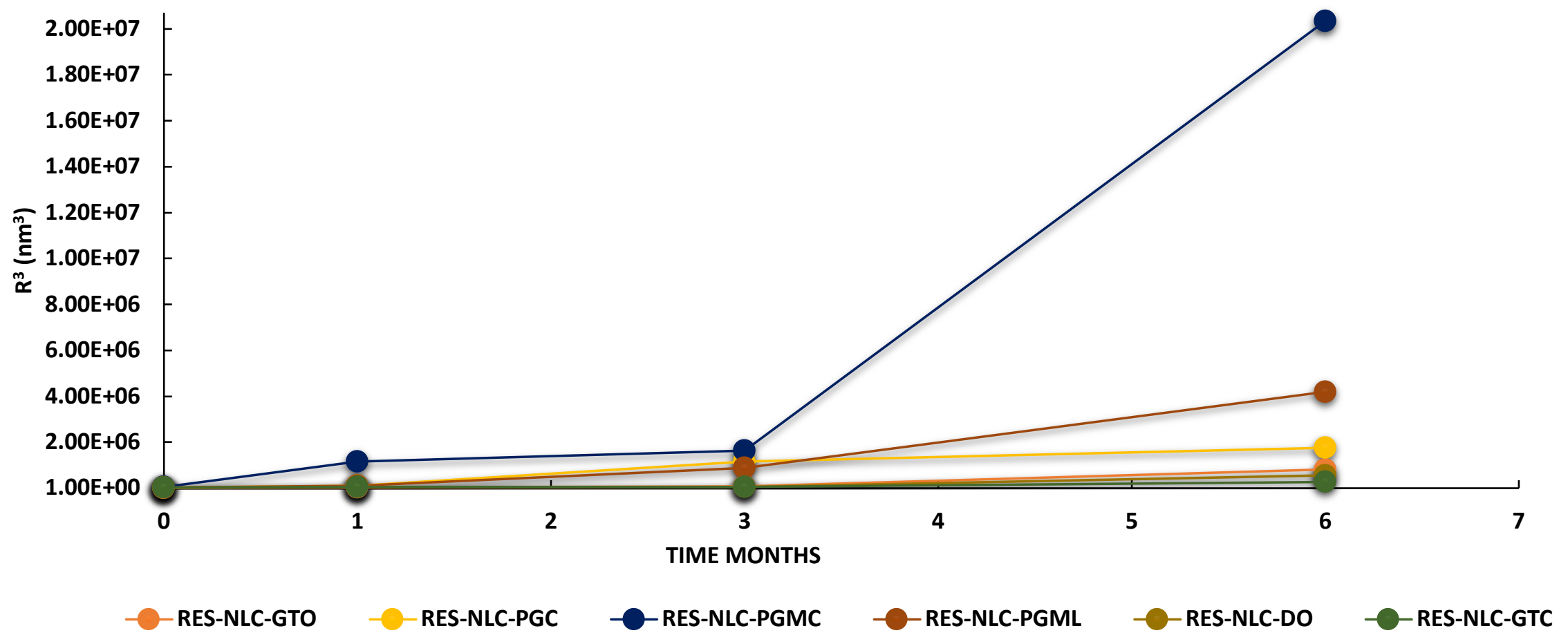




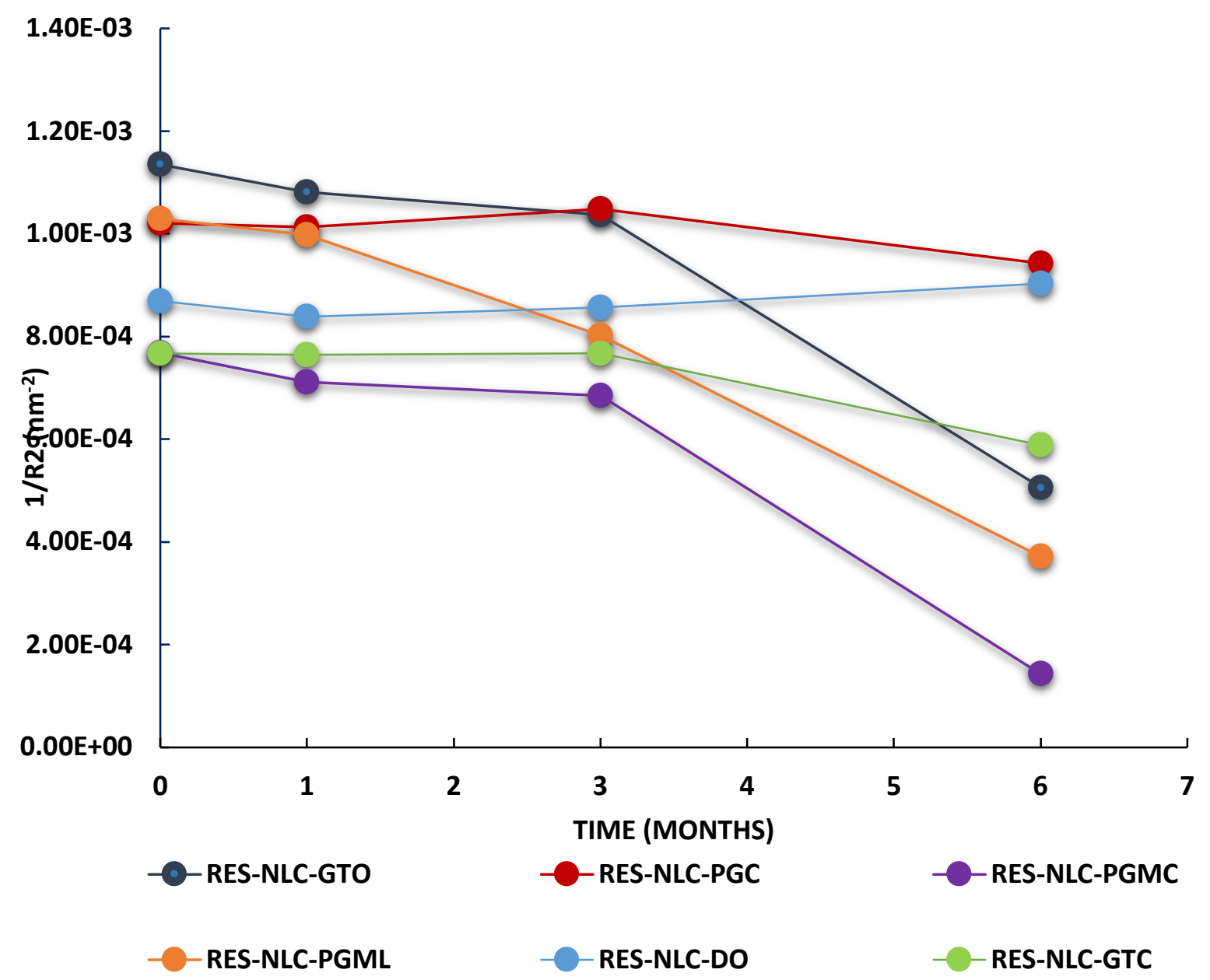


d

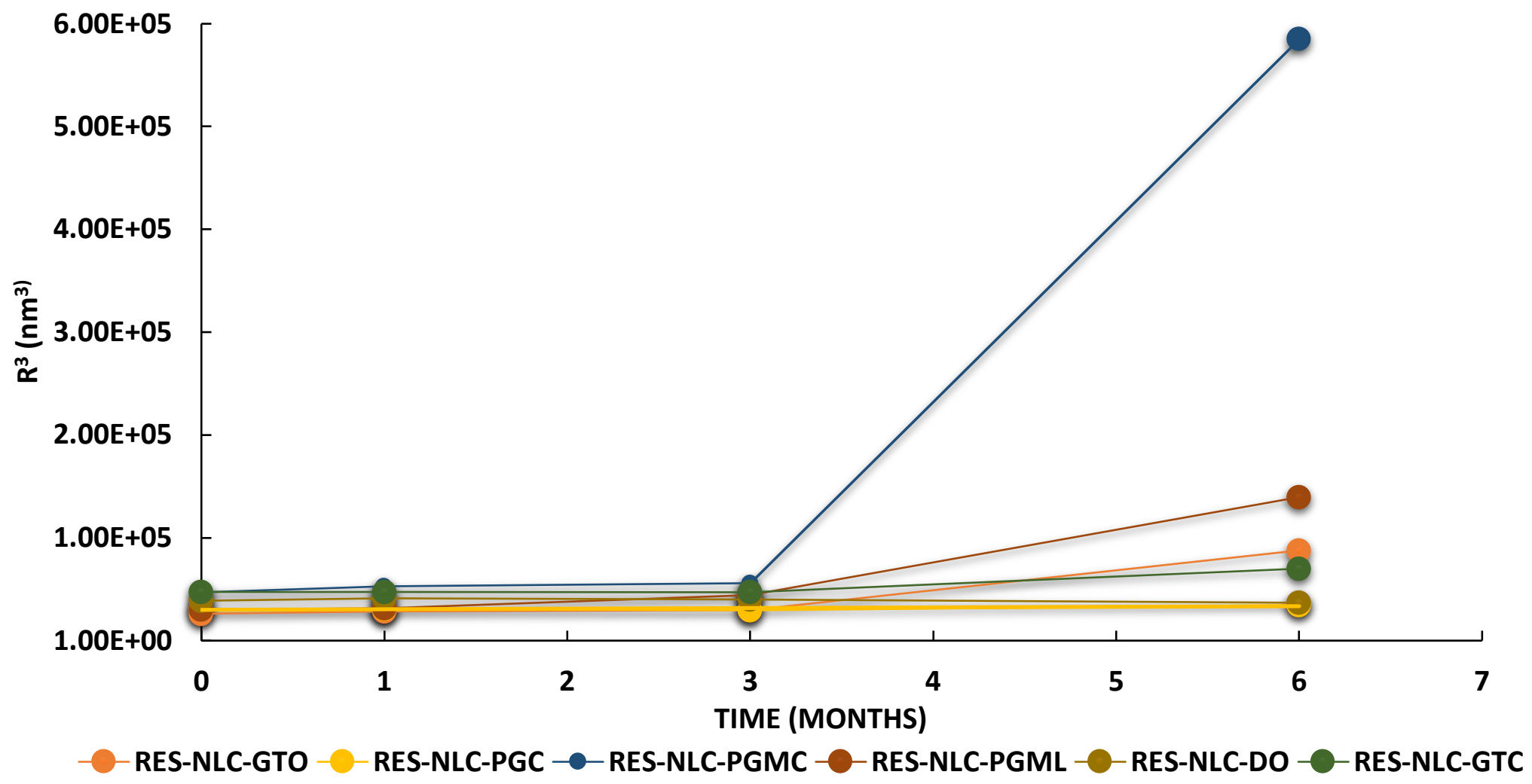

Figure 8) Stability study of NLCs (a) Coalescence force and (b) Ostwald ripening versus time determined for NLCs stored at $4^{\circ} \mathrm{C}$, (c) Coalescence force and (d) Ostwald ripening versus time determine 
Tables

Table 1: Physicochemical properties of liquid lipids and Resveratrol

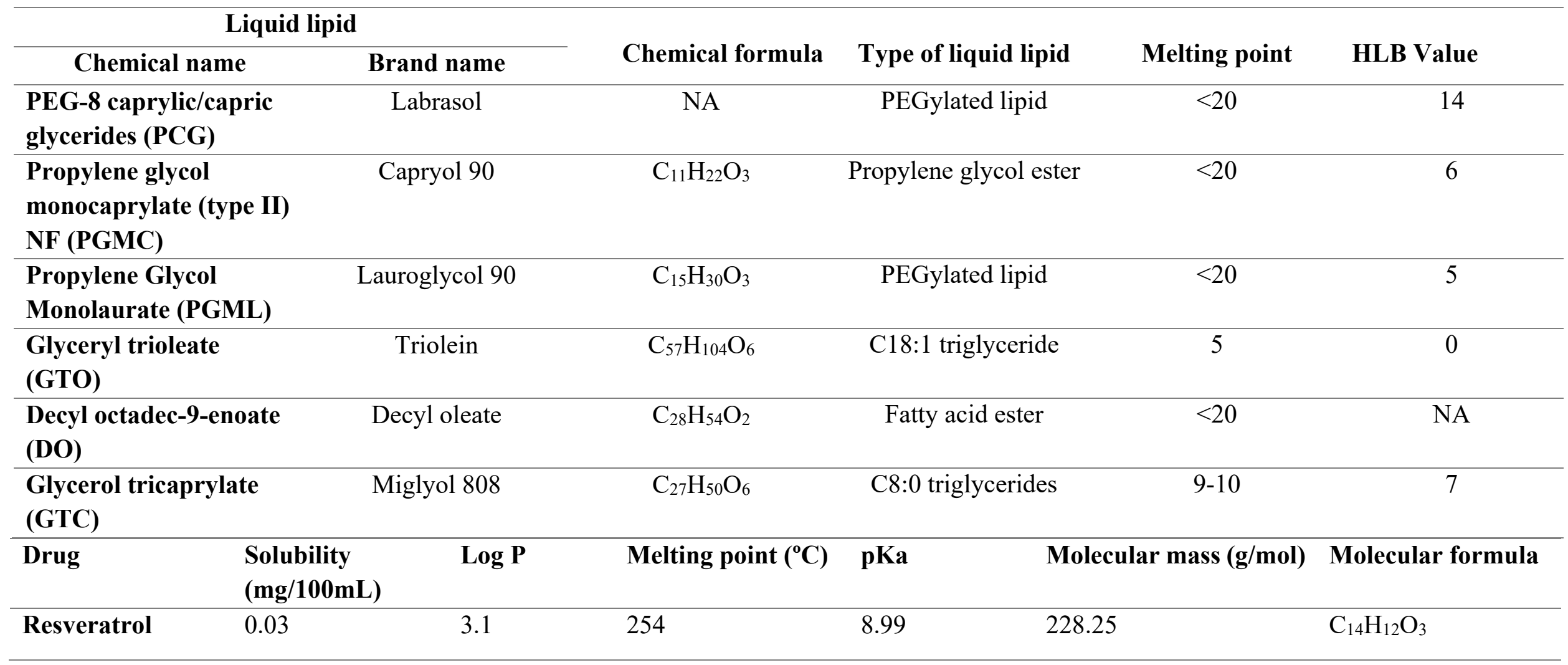


Table 2: Experimental factors and levels in the Box-Behnken design for Resveratrol NLCs

\begin{tabular}{|c|c|c|c|}
\hline \multirow[t]{2}{*}{ Factor } & \multicolumn{3}{|c|}{ Levels Used, Actual (Coded) } \\
\hline & $\begin{array}{l}\text { Low (-1) } \\
(+1)\end{array}$ & Medium (0) & High \\
\hline $\begin{array}{l}\text { A }\left(X_{1}\right)=\text { Concentration of Liquid lipid }(\%) \\
0.75\end{array}$ & & 0.25 & 0.5 \\
\hline $\begin{array}{l}\mathrm{B}\left(\mathrm{X}_{2}\right)=\text { Tween } 80 \text { concentration }(\%) \\
1\end{array}$ & & 0.5 & 0.75 \\
\hline $\begin{array}{l}\mathrm{C}\left(\mathrm{X}_{3}\right)=\text { Amount of drug }(\mathrm{mg}) \\
200\end{array}$ & & 100 & 150 \\
\hline $\begin{array}{l}\text { D= Liquid lipid type } \\
\text { PGML, PCG, DO }\end{array}$ & \multicolumn{3}{|c|}{ GTO, GTC, PGMC, } \\
\hline Dépendent Variables & \multicolumn{3}{|c|}{ Contraints } \\
\hline PS $=$ Particle Size & \multicolumn{3}{|c|}{$<100 \mathrm{~nm}$} \\
\hline PDI= Polydispersity Index & \multicolumn{3}{|c|}{$<0.3$} \\
\hline $\mathrm{ZP}=$ Zeta Potential & \multicolumn{3}{|c|}{ $\pm 30 \mathrm{mV}$} \\
\hline $\mathrm{EE}=$ Entrapment Efficiency & \multicolumn{3}{|c|}{ Maximum } \\
\hline $\mathrm{DL}=$ Drug Loading & \multicolumn{3}{|c|}{ Maximum } \\
\hline
\end{tabular}


Table 3: Analysis of variance (ANOVA) - Lack-of-fit test for each of the response variables

\begin{tabular}{|c|c|c|c|c|c|c|}
\hline Response & Source & $\begin{array}{l}\text { Sum of } \\
\text { squares }\end{array}$ & D.f & $\begin{array}{l}\text { Mean } \\
\text { squares }\end{array}$ & F-value & $\begin{array}{l}p \text { value } \\
(\text { Prob }>\text { F })\end{array}$ \\
\hline \multirow[t]{3}{*}{ Particle size } & Model & 18622.31 & 29 & 642.15 & 4.91 & $0.0001 *$ \\
\hline & Residual & 6283.71 & 48 & 130.91 & & \\
\hline & $\begin{array}{l}\text { Cumulative } \\
\text { Total }\end{array}$ & 24906.02 & 77 & & & \\
\hline \multicolumn{7}{|c|}{ Polydispersity Index } \\
\hline & Model & 0.18 & 29 & $6.216 \mathrm{E}-003$ & 2.58 & $0.0018^{*}$ \\
\hline & Residual & 0.12 & 48 & $2.410 \mathrm{E}-003$ & & \\
\hline & $\begin{array}{l}\text { Cumulative } \\
\text { Total }\end{array}$ & 0.3 & 77 & & & \\
\hline \multicolumn{7}{|c|}{ Zeta potential } \\
\hline & Model & 2835.60 & 29 & 97.78 & 0.85 & 0.6797 \\
\hline & Residual & 5545.04 & 48 & 115.52 & & \\
\hline & $\begin{array}{l}\text { Cumulative } \\
\text { Total }\end{array}$ & 8380.64 & 77 & & & \\
\hline \multicolumn{7}{|l|}{$\begin{array}{l}\text { Entrapment } \\
\text { Efficiency }\end{array}$} \\
\hline & Model & 168.41 & 29 & 5.81 & 4.79 & $<0.0001 *$ \\
\hline & Residual & 58.22 & 48 & 1.21 & & \\
\hline & $\begin{array}{l}\text { Cumulative } \\
\text { Total }\end{array}$ & 226.63 & 77 & & & \\
\hline \multicolumn{7}{|c|}{ Drug Loading } \\
\hline & Model & 138.14 & 29 & 4.76 & 21.80 & $0.0001 *$ \\
\hline & Residual & 10.49 & 48 & 0.22 & & \\
\hline & $\begin{array}{l}\text { Cumulative } \\
\text { Total }\end{array}$ & 148.63 & 77 & & & \\
\hline
\end{tabular}

*Not significant $\alpha<0.05$ (no lack-of-fit) 
Table 4: Compositions of the optimized Resveratrol NLCs formulations for the long-term stability studies

\begin{tabular}{|l|c|c|}
\hline Formulation & $\begin{array}{c}\text { Liquid lipid concentration } \\
(\%)\end{array}$ & $\begin{array}{c}\text { Tween } 80 \text { concentration } \\
(\%)\end{array}$ \\
\hline of the total mixture of lipids & 1 \\
\hline RES-NLC-PCG & 0.5 & 0.75 \\
\hline RES-NLC-PGMC & 0.25 & 0.75 \\
\hline RES-NLC-PGML & 0.25 & 1 \\
\hline RES-NLC-DO & 0.5 & 1 \\
\hline RES-NLC-GTC & 0.5 & 1 \\
\hline
\end{tabular}




\section{SUPPLEMENTARY DATA}

Desirability

- Design Points

П1.000

0.000
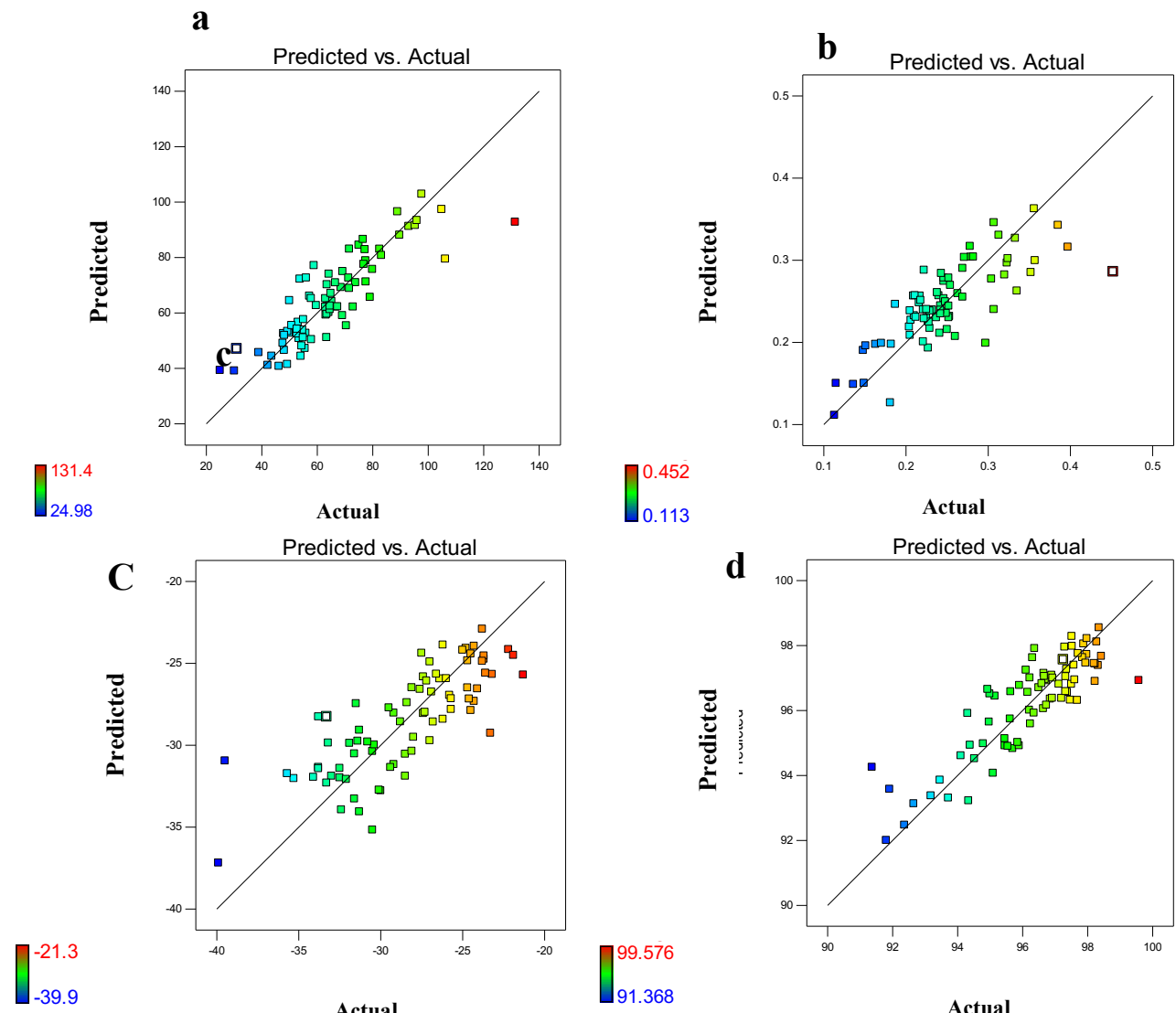

E

Predicted vs. Actual

Actual
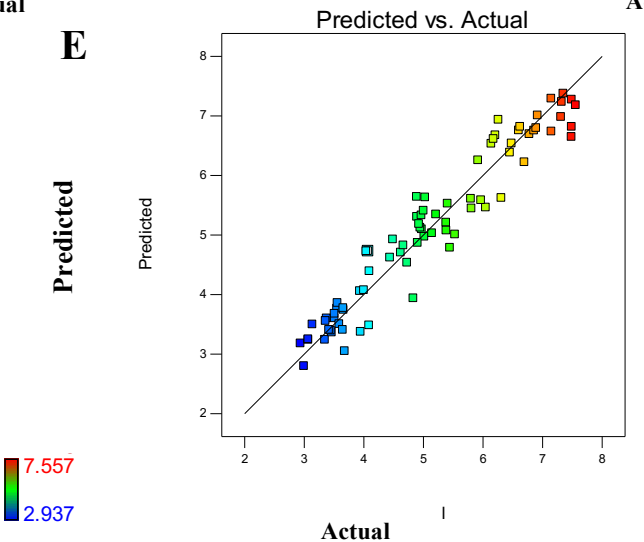

Figure S1: Linear correlation plots between the actual and the predicted values of various responses a. PS, b. PDI, c. ZP, d. EE and e. DL 
a

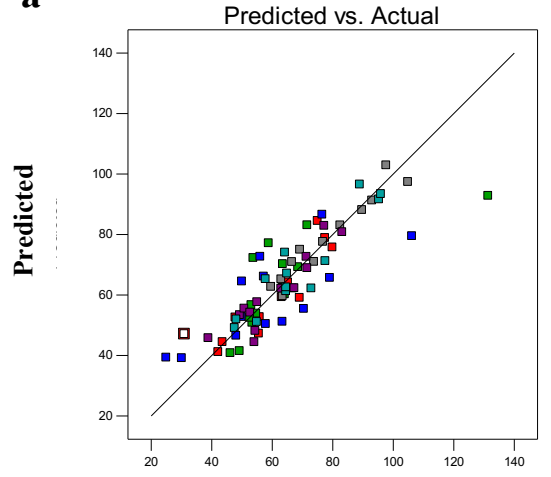

Actual

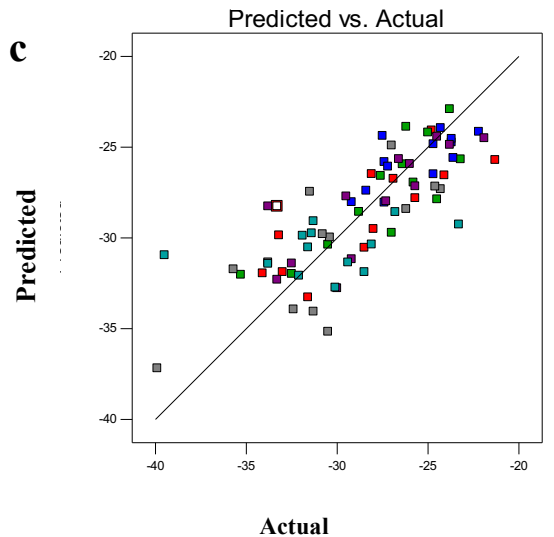

Actual b

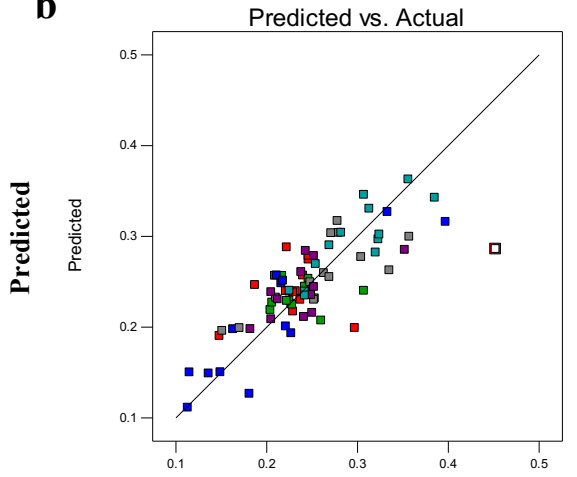

Actual

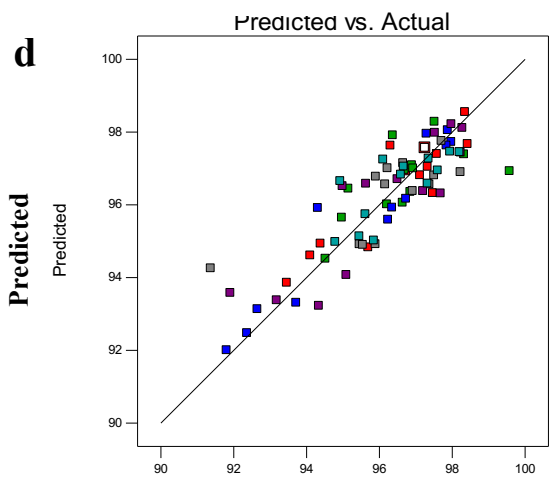

d

Predicted vs. Actual Actual

:

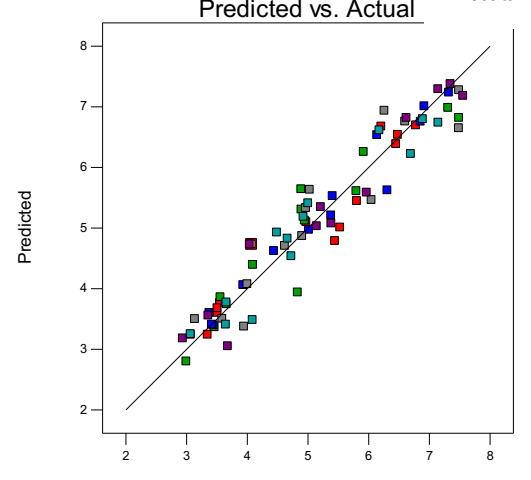

Actual ual

Figure S2: Linear correlation plots between the actual and the predicted values of different types of liquid lipids on different response variables of NLCs a. PS, b. PDI, c. ZP, d. EE and e. DL. Type of liquid lipid: GTC, DO, PCG, PGMC, GTO and PGML 

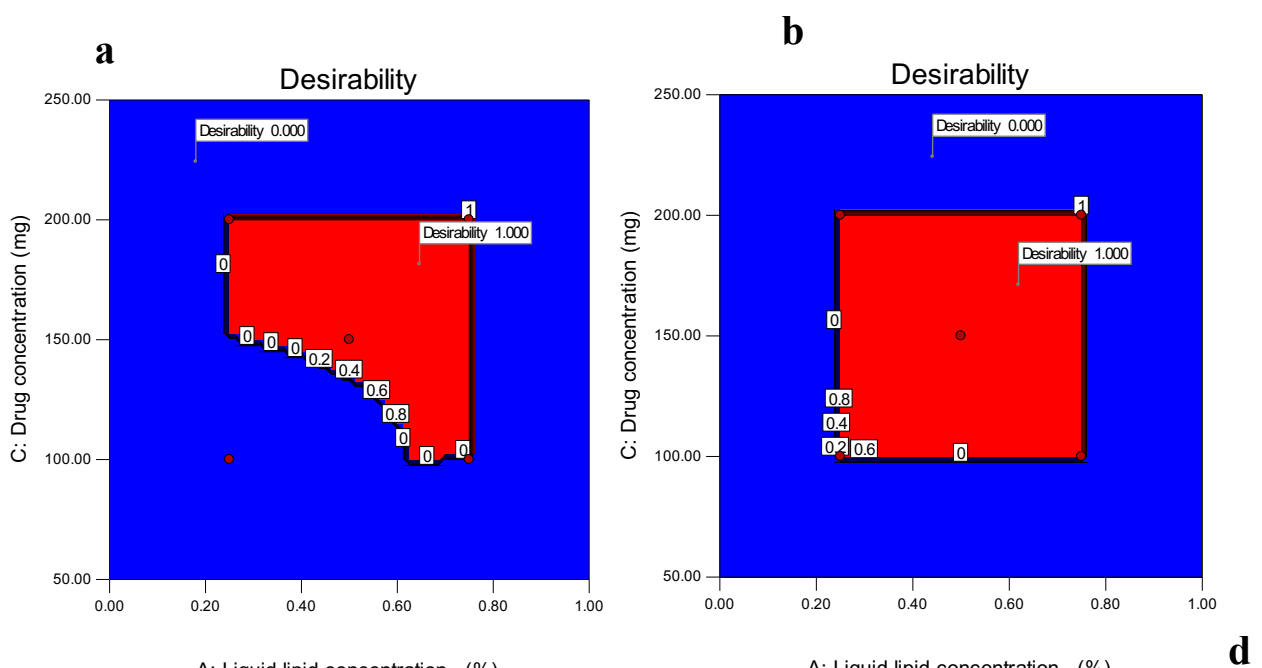

A: Liquid lipid concentration (\%)

Desirability
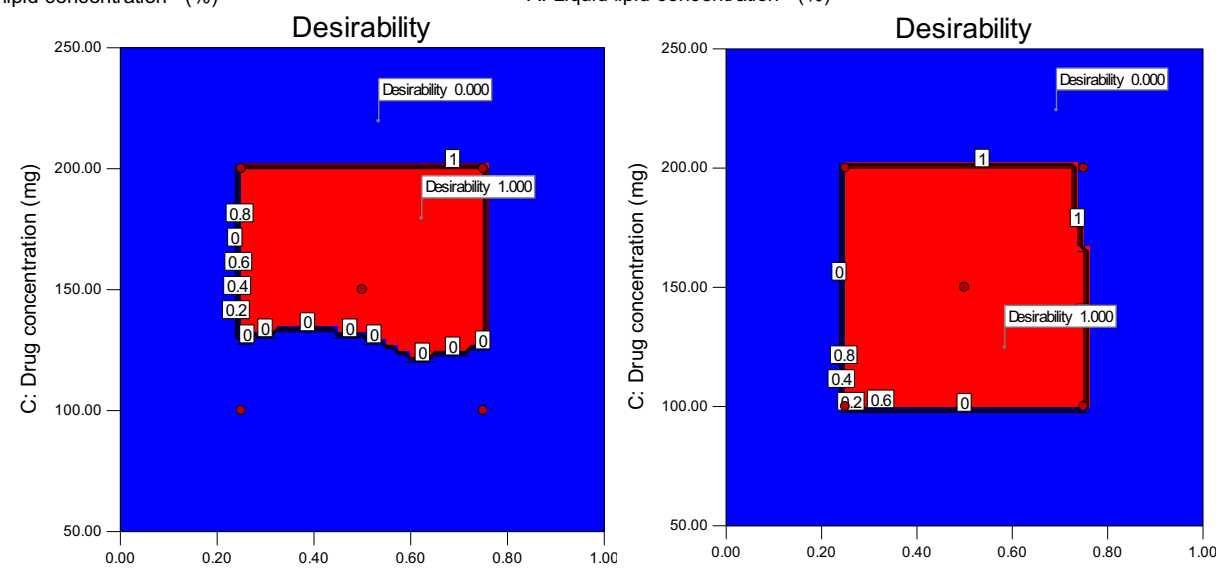

A: Liquid lipid concentration (\%)

e
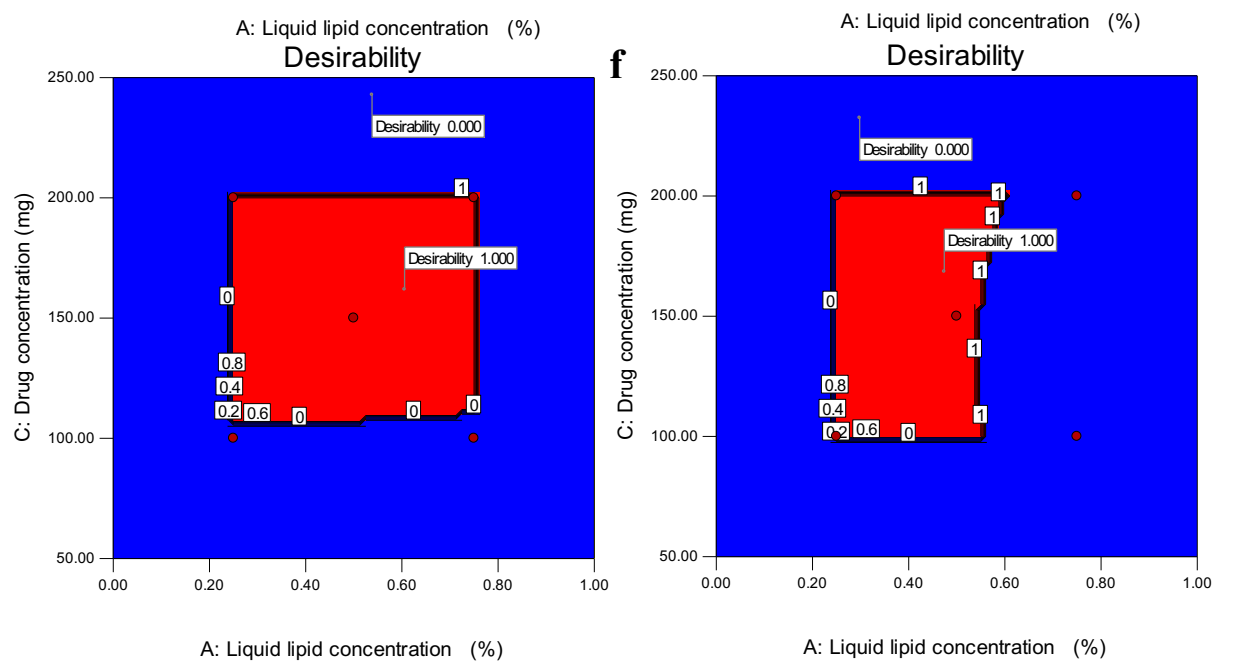

Figure S3: Desirability of the design space for different liquid lipid types at medium Tween 80 concnetration a: GTC, b: DO, c: PCG, d: PGMC e: GTO f: PGML 

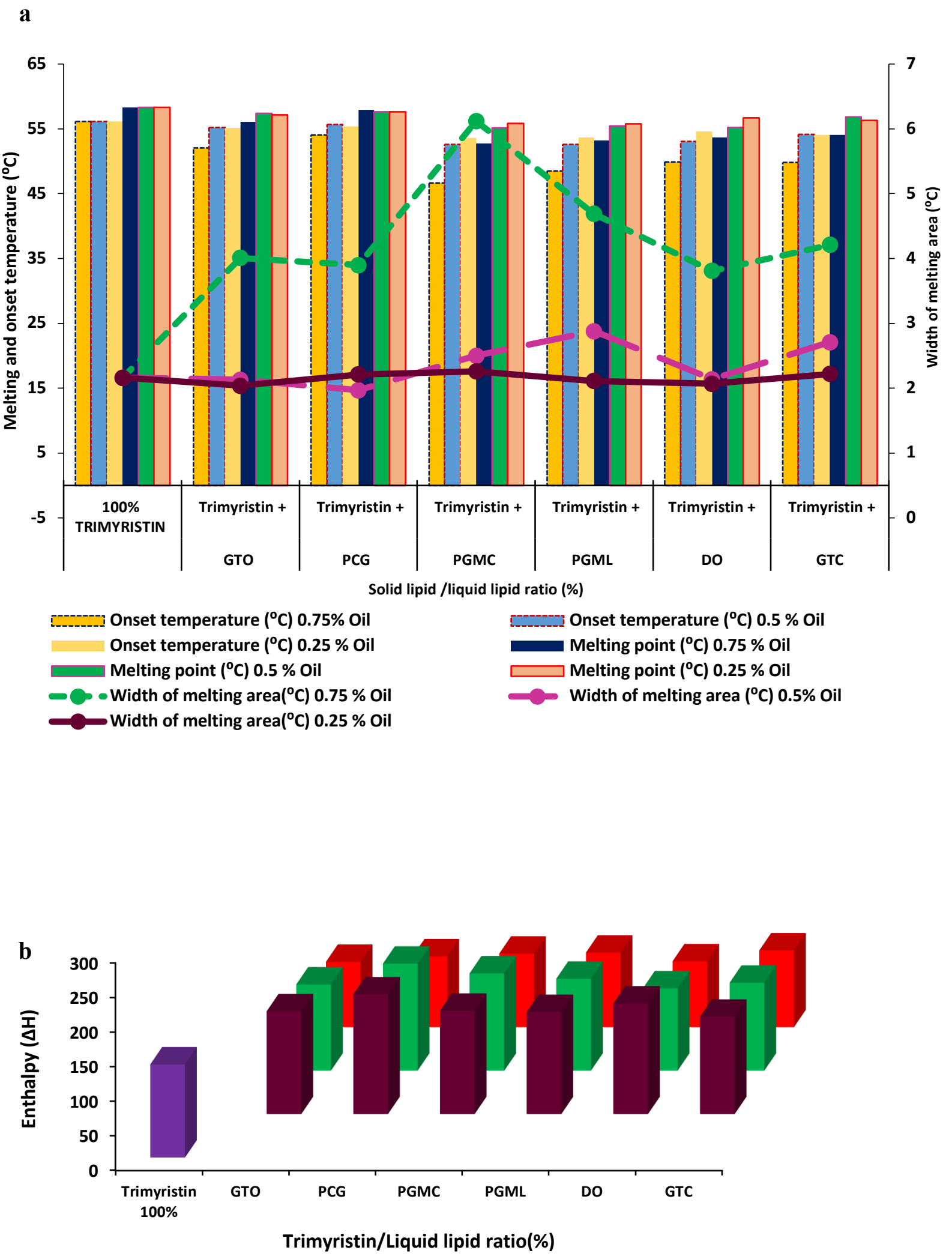

$\square 0.25 \%$ Liquid lipid $\quad \square .5 \%$ Liquid lipid $\quad \square 0.75 \%$ Liquid lipid

Figure S4. Bar graph representing a. Decrease of melting point, onset temperature and increase in the width of melting area of solid lipid with different concentrations of liquid lipids and b. Overlay of the melting enthalpy of solid lipid mixture with all liquid lipid concentration 


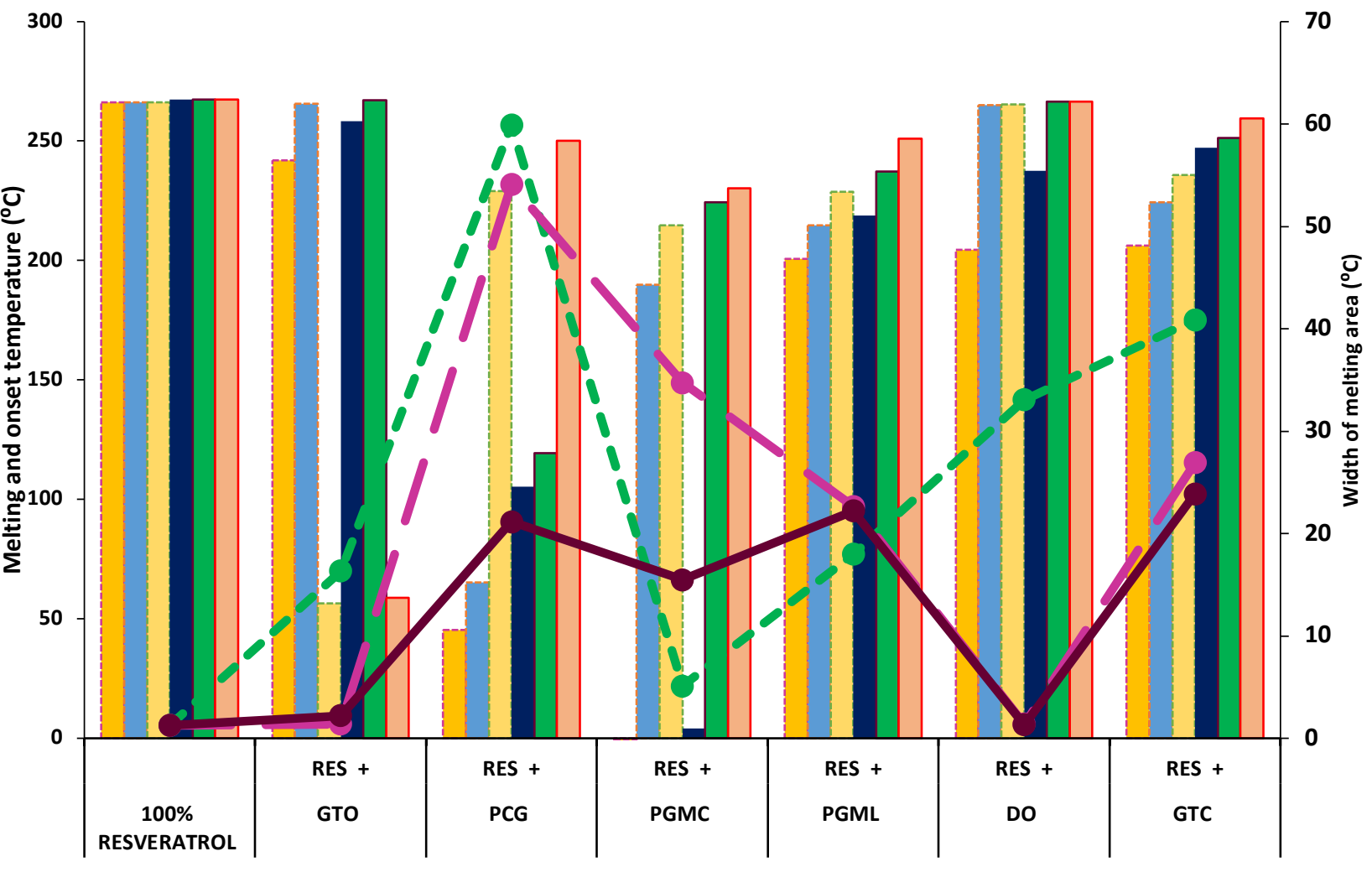

Resvertrol/liquid lipid ratio (\%)

Onset temperature $\left({ }^{\circ} \mathrm{C}\right) 0.75 \%$ Oil

Onset temperature $\left({ }^{\circ} \mathrm{C}\right) 0.25 \%$ oll

Melting point $\left({ }^{\circ} \mathrm{C}\right) 0.5 \%$ Oil

Width of melting area $\left({ }^{\circ} \mathrm{C}\right) 0.75 \%$ Oil

Width of melting area $\left({ }^{\circ} \mathrm{C}\right) 0.25 \%$ olL

Onset temperature $\left({ }^{\circ} \mathrm{C}\right) 0.5 \%$ Oil

Melting point $\left({ }^{\circ} \mathrm{C}\right) 0.75 \%$ Oil

$\square$ Melting point $\left({ }^{\circ} \mathrm{C}\right) 0.25 \%$ oil

Width of melting area $\left({ }^{\circ} \mathrm{C}\right) 0.5 \%$ Oil

b

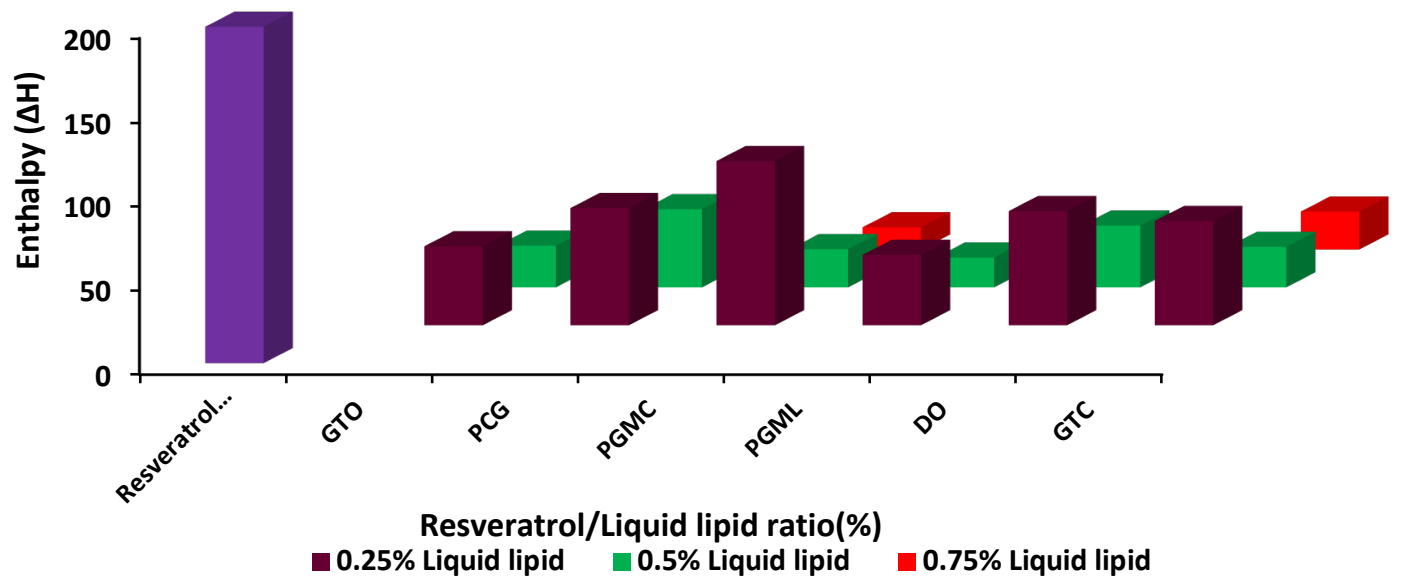

Figure S5. Bar graph representing a. Decrease of melting point, onset temperature and increase in the width of melting area of RES with different concentrations of liquid lipids and b. Overlay of the melting enthalpy of RES mixture with all liquid lipid concentration 
Table S1: $\mathbf{R}^{2}$ values for determining coalescence and Ostwald ripening mechanism for particle growth for six different RES-NLCs at both $4^{\circ} \mathrm{C}$ and $20^{\circ} \mathrm{C}$

\begin{tabular}{|c|c|c|c|c|}
\hline \multicolumn{3}{|c|}{$4^{\circ} \mathrm{C}$ storage temperature } & \multicolumn{2}{|c|}{$20^{\circ} \mathrm{C}$ storage temperature } \\
\hline Formulations & $\begin{array}{l}\text { Coalescence } \\
R^{2} \text { value for } \\
1 / r^{2} \text { vs } t \text { plot }\end{array}$ & $\begin{array}{l}\text { Ostwald } \\
\text { ripening } \\
R^{2} \text { value for } \\
\mathbf{r}^{3} \text { vs t plot }\end{array}$ & $\begin{array}{l}\text { Coalescence } \\
R^{2} \text { value for } \\
1 / r^{2} \text { vs } t \text { plot }\end{array}$ & $\begin{array}{l}\text { Ostwald ripening } \\
R^{2} \text { value for } \\
\mathbf{r}^{3} v \text { s t plot }\end{array}$ \\
\hline $\begin{array}{l}\text { RES-NLC- } \\
\text { GTO }\end{array}$ & 0.909 & 0.839 & 0.877 & 0.818 \\
\hline $\begin{array}{l}\text { RES-NLC- } \\
\text { PCG }\end{array}$ & 0.662 & 0.951 & 0.486 & 0.519 \\
\hline $\begin{array}{l}\text { RES-NLC- } \\
\text { PGMC }\end{array}$ & 0.495 & 0.827 & 0.860 & 0.788 \\
\hline $\begin{array}{l}\text { RES-NLC- } \\
\text { PGML }\end{array}$ & 0.722 & 0.912 & 0.970 & 0.864 \\
\hline $\begin{array}{l}\text { RES-NLC- } \\
\text { DO }\end{array}$ & 0.792 & 0.815 & 0.526 & 0.509 \\
\hline $\begin{array}{l}\text { RES-NLC- } \\
\text { GTC }\end{array}$ & 0.925 & 0.878 & 0.772 & 0.774 \\
\hline
\end{tabular}

